Supporting Information for

\title{
Catalytic Asymmetric Cascade Cyclization for Constructing Three Contiguous Stereocenters in Pyrrolobenzodiazepine-Based Cyclopentanones
}

Zhao Wei, ${ }^{[a, b]}$ Jinlong Zhang, ${ }^{[a]}$ Huameng Yang, ${ }^{[a]}$ and Gaoxi Jiang*[a]

aState Key Laboratory for Oxo Synthesis and Selective Oxidation, Center for Excellence in Molecular Synthesis, Suzhou Research Institute of LICP, Lanzhou Institute of Chemical Physics (LICP), Chinese Academy of Sciences, Lanzhou 730000, P. R. China

${ }^{\mathrm{b}}$ University of Chinese Academy of Sciences, Beijing 100049, P. R. China

E-mail: gxjiang@licp.cas.cn

Table of Contents

1 General experimental materials $\quad$ S2

2 General procedure for the reactions $\quad \mathbf{S 3}$

3 Experimental characterization data for new products $\quad$ S5

$4{ }^{1} \mathrm{H}$ and ${ }^{13} \mathrm{C}$ NMR spectra for all compounds $\quad$ S15

$5 \quad$ HPLC analysis and spectra $\quad$ S43 


\section{General experimental materials}

Experimental: Unless otherwise noted, all solvents were dried with sodium, benzophenone and distilled before use. All reactions were set up under $\mathrm{N}_{2}$ utilizing glassware that was flame-dried and cooled under vacuum. All non-aqueous manipulations were using standard Schlenk techniques. Reactions were monitored using thin-layer chromatography (TLC) on Silica Gel plates. Visualization of the developed plates was performed under UV light $(254 \mathrm{~nm})$ or phosphomolybdic acid hydrate stain. Silica-gel flash column chromatography was performed on Adamas 100-200 mesh silica gel.

Materials: Unless otherwise indicated, starting catalysts and materials were obtained from Sigma Aldrich, TCI, Alfa Aesar, or Acros Co. Ltd. Moreover, commercially available reagents were used without additional purification.2-(1H-pyrrol-1-yl)aniline and furylcarbinol with different substituents were prepared according to literature procedures.

Instrumentation: All NMR spectra were run at $400 \mathrm{MHz}\left({ }^{1} \mathrm{H} \mathrm{NMR}\right)$ or $100 \mathrm{MHz}\left({ }^{13} \mathrm{C} \mathrm{NMR}\right)$ in $\mathrm{CDCl}_{3}$ solution. ${ }^{1} \mathrm{H}$ NMR spectra were internally referenced to TMS. $13 \mathrm{C}$ NMR spectra were internally referenced to the residual solvent signal. Data for ${ }^{1} \mathrm{H}$ NMR are reported as follows: chemical shift $(\delta \mathrm{ppm})$, multiplicity $(\mathrm{s}=$ singlet, $\mathrm{d}=$ doublet, $\mathrm{t}=$ triplet, $\mathrm{q}=$ quartet, $\mathrm{m}=$ multiplet, br $=$ broad), coupling constants $(J)$ were reported in Hz. High resolution mass spectra (HRMS) were recorded on Angilent Q-TOF LC/MS mass instrument (ESI). The enantiomeric excesses were determined by HPLC analysis which employing chiral stationary phase column specified in the individual experiment, by comparing the samples with the appropriate racemic mixtures. 


\section{General procedure for the reaction}

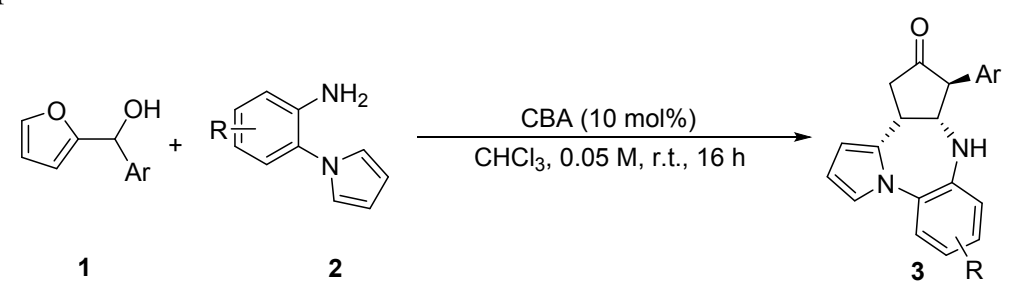

A dry reaction tube equipped with a stir bar was charged with 1-(2-Aminophenyl)Pyrrole 2 ( $0.1 \mathrm{mmol}, 1.0$ equiv.), CBA (10 mol\%, $4.5 \mathrm{mg})$ and $50 \mathrm{mg} 5 \AA$ molecular sieves. It was capped with a rubber septum, evacuated and backfilled with nitrogen. Then, furylcarbinol $1(0.15 \mathrm{mmol}$, 1.5 equiv.) dissolved in $\mathrm{CHCl}_{3}(2 \mathrm{~mL})$ was added. The reaction mixture was stirred at $25{ }^{\circ} \mathrm{C}$ for 16 hours. Upon completion, the mixture was directly subjected to silica gel flash chromatography to give the pure product.

1.0-mmol scale reaction for 4oj:

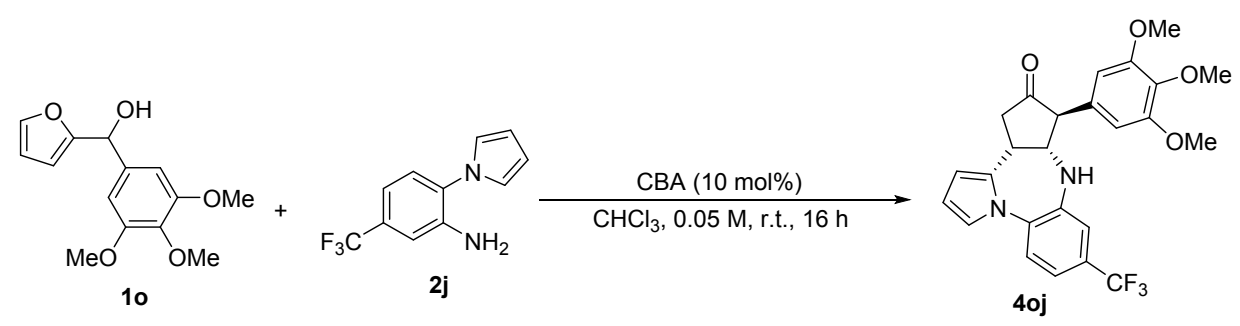

A dry reaction tube equipped with a stir bar was charged with $2 \mathbf{j}$ ( $1.0 \mathrm{mmol}, 226 \mathrm{mg}, 1.0$ equiv.), CBA (10 mol\%, $45 \mathrm{mg}$ ) and $500 \mathrm{mg} 5 \AA$ molecular sieves. It was capped with a rubber septum, evacuated and backfilled with nitrogen. Then, 10 (1.5 mmol, $396 \mathrm{mg}, 1.5$ equiv.) dissolved in $\mathrm{CHCl}_{3}$ $(20 \mathrm{~mL})$ was added. The reaction mixture was stirred at $25^{\circ} \mathrm{C}$ for 16 hours. Upon completion, the mixture was purified by silica gel flash chromatography to give the pure product 4oj in $425 \mathrm{mg}$ (90\% yield).

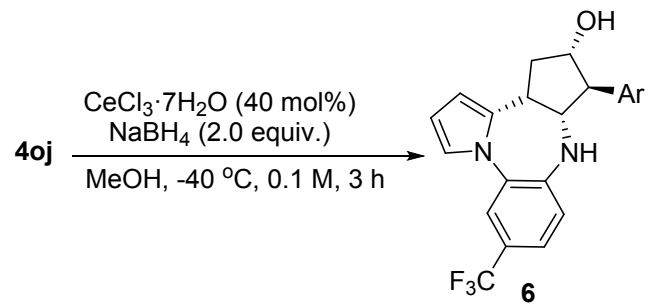

4oj $(0.2 \mathrm{mmol})$ was added to a dried Schlenk tube followed by added $\mathrm{MeOH}(2 \mathrm{~mL})$ under $\mathrm{N}_{2}$ atmosphere at $-40{ }^{\circ} \mathrm{C}$, then $\mathrm{CeCl}_{3} \cdot 7 \mathrm{H}_{2} \mathrm{O}$ (0.4 equiv.) and $\mathrm{NaBH}_{4}(2.0$ equiv.) was added. The reaction mixture was stirred for $3 \mathrm{~h}$. The reaction mixture was quenched with saturated aqueous $\mathrm{NH}_{4} \mathrm{Cl}(5 \mathrm{~mL})$ and extract with ethyl acetate $(3 \times 5 \mathrm{~mL})$. The combined organic layers were dried with $\mathrm{Na}_{2} \mathrm{SO}_{4}$, filtered and then concentrated in vacuo, purified by flash column chromatography (PE:EA = 2:1) to afford $\mathbf{6}$ as white foam (54.2 mg, 78\% yield). 


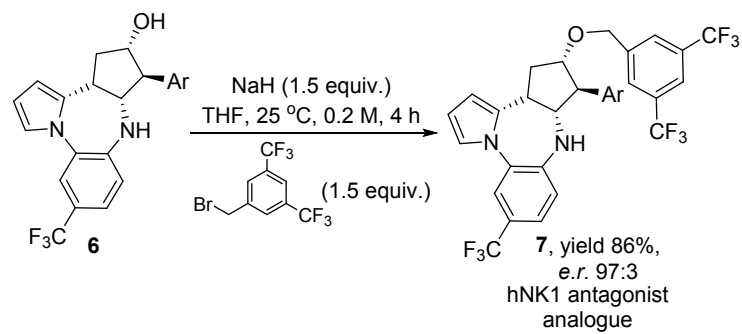

Chiral cyclopentanol $6(0.15 \mathrm{mmol})$ was added slowly to a suspension of $\mathrm{NaH}$ (4.5 $\mathrm{mg}, 1.2$ equiv.) in $2 \mathrm{~mL}$ anhydrous THF under an atmosphere of $\mathrm{N}_{2}$ and stirred for $1 \mathrm{~h}$. Subsequently, 3,5bistrifluoromethylbenzyl bromide $(0.23 \mathrm{mmol}, 1.5$ equiv.) was added to the solution and the reaction was stirred at r.t. for $4 \mathrm{~h}$. The reaction was quenched with saturated aqueous $\mathrm{NH}_{4} \mathrm{Cl}(5 \mathrm{~mL})$ and extract with ethyl acetate $(3 \times 5 \mathrm{~mL})$. The combined organic layers were dried with $\mathrm{Na}_{2} \mathrm{SO}_{4}$, filtered and then concentrated in vacuo, purified by flash column chromatography (PE:EA $=4: 1)$ to afford 7 as pale yellow solid (73.9 mg, 86\% yield). 


\section{Experimental characterization data for new products}

(9aR,10S,12aR)-10-phenyl-9a,10,12,12a-tetrahydrobenzo[b]cyclopenta[f]pyrrolo [1,2-d][1,4]diazepin-

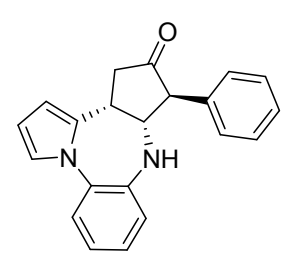

3aa

11(9H)-one (3aa):

White foamy solid after flash chromatography $(\mathrm{PE}: \mathrm{EA}=10: 1), 28.9 \mathrm{mg}, 92 \%$ yield. The enantiomeric purity of the product was determined by HPLC analysis: er 94:6 (Chiralcel OD-H, hexane $/ i-\mathrm{PrOH}=70: 30$, flow rate $1.0 \mathrm{~mL} / \mathrm{min}, \lambda=254 \mathrm{~nm}$ ), $t_{\mathrm{r}}$ (major) $=17.177 \mathrm{~min}, t_{\mathrm{r}}$ (minor) $=42.225 \mathrm{~min} .{ }^{1} \mathrm{H}$ NMR (400 MHz, Chloroformd) $\delta 7.39-7.30(\mathrm{~m}, 4 \mathrm{H}), 7.18-7.12(\mathrm{~m}, 4 \mathrm{H}), 6.98(\mathrm{~s}, 1 \mathrm{H}), 6.90-6.84(\mathrm{~m}, 1 \mathrm{H}), 6.30-$ $6.27(\mathrm{~m}, 1 \mathrm{H}), 6.19-6.13(\mathrm{~m}, 1 \mathrm{H}), 4.36-4.27(\mathrm{~m}, 1 \mathrm{H}), 3.65(\mathrm{~d}, J=9.8 \mathrm{~Hz}, 2 \mathrm{H}), 3.55(\mathrm{~s}, 1 \mathrm{H}), 3.02(\mathrm{~d}, J=$ $19.0 \mathrm{~Hz}, 1 \mathrm{H}), 2.71$ (dd, $J=19.2,8.5 \mathrm{~Hz}, 1 \mathrm{H}) .{ }^{13} \mathrm{C}$ NMR see: B. V. Reddy, Y. V. Reddy, K. K. Singarapu, Org. Biomol. Chem. 2016, 14, 1111-1116. HRMS (ESI) calcd. for $\mathrm{C}_{21} \mathrm{H}_{19} \mathrm{~N}_{2} \mathrm{O}$ [M+H]: 315.1497, found: 315.1492 .

(9aR,10S,12aR)-10-(o-tolyl)-9a,10,12,12a-tetrahydrobenzo[b]cyclopenta[f]pyrrolo[1,2-d][1,4]diazepin

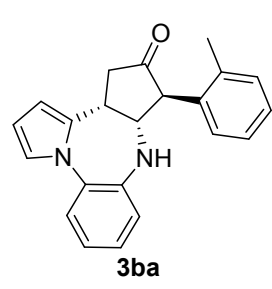
-11(9H)-one (3ba):

White foamy solid after flash chromatography (PE:EA=10:1), $26.6 \mathrm{mg}, 81 \%$ yield. The enantiomeric purity of the product was determined by HPLC analysis: $\mathrm{er}$ 90.5:9.5 (Chiralcel IA, hexane $/ i$ - $\mathrm{PrOH}=70: 30$, flow rate $1.0 \mathrm{~mL} / \mathrm{min}, \lambda=254 \mathrm{~nm}$ ), $t_{\mathrm{r}}($ major $)=7.195 \mathrm{~min}, t_{\mathrm{r}}($ minor $)=6.475 \mathrm{~min} .{ }^{1} \mathrm{H}$ NMR $(400 \mathrm{MHz}$, Chloroformd): $\delta 7.41-7.33(\mathrm{~m}, 1 \mathrm{H}), 7.24-7.19(\mathrm{~m}, 3 \mathrm{H}), 7.16-7.11(\mathrm{~m}, 2 \mathrm{H}), 7.07-6.99(\mathrm{~m}$, $1 \mathrm{H}), 6.98(\mathrm{t}, J=2.4 \mathrm{~Hz}, 1 \mathrm{H}), 6.94-6.86(\mathrm{~m}, 1 \mathrm{H}), 6.30(\mathrm{t}, J=3.3 \mathrm{~Hz}, 1 \mathrm{H}), 6.21-6.15(\mathrm{~m}, 1 \mathrm{H}), 4.45(\mathrm{dd}$, $J=11.0,6.4 \mathrm{~Hz}, 1 \mathrm{H}), 3.94(\mathrm{~d}, J=10.8,1 \mathrm{H}), 3.68(\mathrm{t}, J=7.4 \mathrm{~Hz}, 1 \mathrm{H}), 3.53(\mathrm{~s}, 1 \mathrm{H}), 3.02(\mathrm{~d}, J=19.0 \mathrm{~Hz}$, 1H), 2.74 (dd, $J=19.0,8.5 \mathrm{~Hz}, 1 \mathrm{H}), 2.26$ (s, 3H). ${ }^{13}$ C NMR (100 MHz, Chloroform-d): $\delta 214.03$, 
$139.17,138.04,134.78,133.92,131.15,131.06,127.61,127.13,126.58,124.15,123.42,122.57,121.57$, 108.52, 106.53, 69.95, 41.66, 33.85, 20.23. HRMS (ESI) calcd. for $\mathrm{C}_{22} \mathrm{H}_{21} \mathrm{~N}_{2} \mathrm{O}[\mathrm{M}+\mathrm{H}]: 329.1654$, found: 329.1659 .

(9aR,10S,12aR)-10-(2-fluorophenyl)-9a,10,12,12a-tetrahydrobenzo[b]cyclopenta[f]pyrrolo[1,2-d][1,4]diazepin-11(9H)-one (3ca):

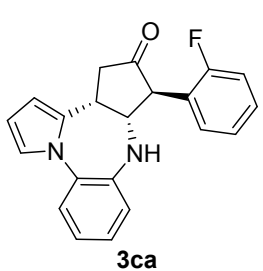

White foamy solid after flash chromatography (PE:EA = 10:1), $31.2 \mathrm{mg}, 94 \%$ yield. The enantiomeric purity of the product was determined by HPLC analysis: er 93:7 (Chiralcel IA, hexane $/ i-\mathrm{PrOH}=70: 30$, flow rate $1.0 \mathrm{~mL} / \mathrm{min}, \lambda=360 \mathrm{~nm}$ ), $t_{\mathrm{r}}$ (major) $=8.383 \mathrm{~min}, t_{\mathrm{r}}$ (minor) $=6.619 \mathrm{~min} .{ }^{1} \mathbf{H}$ NMR $(400 \mathrm{MHz}$, Chloroform- $\boldsymbol{d}) \delta 7.40-$ 7.29 (m, 2H), 7.19-7.07 (m, 5H), 7.01-6.97 (m, 1H), 6.95-6.90 (m, 1H), 6.35-6.25 $(\mathrm{m}, 1 \mathrm{H}), 6.21-6.12(\mathrm{~s}, 1 \mathrm{H}), 4.52-4.37(\mathrm{~s}, 1 \mathrm{H}), 3.75-3.62(\mathrm{~m}, 2 \mathrm{H}), 3.51(\mathrm{~s}, 1 \mathrm{H}), 3.00$ $(\mathrm{d}, J=18.8 \mathrm{~Hz}, 1 \mathrm{H}), 2.84(\mathrm{dd}, J=18.7,8.0 \mathrm{~Hz}, 1 \mathrm{H}) .{ }^{13} \mathbf{C}$ NMR (100 MHz, Chloroform-d) $\delta 212.92$, $160.94(\mathrm{~d}, \quad J=245.2 \mathrm{~Hz}), 139.03,133.85,132.22$, (d, $J=4.9 \mathrm{~Hz}), 130.86,129.75(\mathrm{~d}, J=8.3 \mathrm{~Hz}), 127.17$, $124.69,124.25,123.44,122.64,121.60,116.24,116.03,108.60,106.70,68.95,57.40,41.19,34.17$. HRMS (ESI) calcd. for $\mathrm{C}_{21} \mathrm{H}_{18} \mathrm{FN}_{2} \mathrm{O}[\mathrm{M}+\mathrm{H}]: 333.1403$, found: 333.1406 .

(9aR,10S,12aR)-10-(m-tolyl)-9a,10,12,12a-tetrahydrobenzo[b]cyclopenta[f]pyrrolo[1,2-d][1,4]diazepin $-11(9 \mathrm{H})$-one (3da):

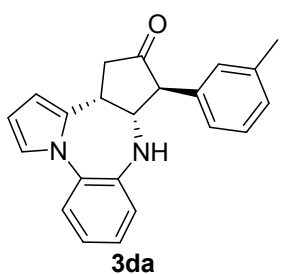

White foamy solid after flash chromatography $(\mathrm{PE}: \mathrm{EA}=10: 1), 31.4 \mathrm{mg}, 95 \%$ yield. The enantiomeric purity of the product was determined by HPLC analysis: er 92:8 (Chiralcel IA, hexane $/ i-\mathrm{PrOH}=70: 30$, flow rate $1.0 \mathrm{~mL} / \mathrm{min}, \lambda=360 \mathrm{~nm}$ ), $t_{\mathrm{r}}($ major $)=8.142 \mathrm{~min}, t_{\mathrm{r}}($ minor $)=6.181 \mathrm{~min} .{ }^{1} \mathrm{H}$ NMR $(400 \mathrm{MHz}$, Chloroformd) $\delta 7.43-7.34(\mathrm{~m}, 1 \mathrm{H}), 7.29-7.22(\mathrm{~m}, 1 \mathrm{H}), 7.19-7.07(\mathrm{~m}, 3 \mathrm{H}), 7.04-6.84(\mathrm{~m}$, 4H), 6.32-6.25 (m, 1H), 6.19-6.12 (m, 1H), $4.36-4.26(\mathrm{~m}, 1 \mathrm{H}), 3.70-3.58(\mathrm{~m}$, 2H), 3.54 (s, 1H), 3.02 (d, $J=18.9 \mathrm{~Hz}, 1 \mathrm{H}), 2.72(\mathrm{dd}, J=19.1,8.4 \mathrm{~Hz}, 1 \mathrm{H}), 2.34(\mathrm{~s}, 3 \mathrm{H}) .{ }^{13} \mathbf{C}$ NMR (100 MHz, Chloroform-d) $\delta 214.35,138.85,136.30,133.89,131.10,129.81,129.05,128.54,125.95,124.19$, 123.45, 122.62, 121.59, 108.56, 70.64, 60.98, 41.78, 33.66, 21.52. HRMS (ESI) calcd. for $\mathrm{C}_{22} \mathrm{H}_{21} \mathrm{~N}_{2} \mathrm{O}$ $[\mathrm{M}+\mathrm{H}]: 329.1654$, found: 329.1659 .

(9aR,10S,12aR)-10-(3-methoxyphenyl)-9a,10,12,12a-tetrahydrobenzo[b]cyclopenta[f]pyrrolo[1,2d][1,4]diazepin-11(9H)-one (3ea):

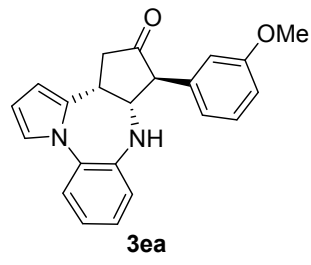

White foamy solid after flash chromatography (PE:EA = 10:1), $32.6 \mathrm{mg}, 95 \%$ yield. The enantiomeric purity of the product was determined by HPLC analysis er 95.5:4.5 (Chiralcel IA, hexane $/ i-\mathrm{PrOH}=70: 30$, flow rate $1.0 \mathrm{~mL} / \mathrm{min}, \lambda=$ $254 \mathrm{~nm}), t_{\mathrm{r}}($ major $)=10.899 \mathrm{~min}, t_{\mathrm{r}}($ minor $)=8.008 \mathrm{~min} .{ }^{1} \mathbf{H} \mathbf{~ N M R}(400 \mathrm{MHz}$, Chloroform- $\boldsymbol{d}) \delta$ 7.39-7.33 (m, 1H), 7.33-7.26 (m, 1H), 7.18-7.11 (m, 2H), $6.99(\mathrm{~s}, 1 \mathrm{H}), 6.94-6.88(\mathrm{~m}, 1 \mathrm{H}), 6.88-6.83(\mathrm{~m}, 1 \mathrm{H}), 6.74-6.66(\mathrm{~m}, 2 \mathrm{H}), 6.32-$ 
$6.27(\mathrm{~m}, 1 \mathrm{H}), 6.18-6.13(\mathrm{~m}, 1 \mathrm{H}), 4.37-4.28(\mathrm{~m}, 1 \mathrm{H}), 3.78(\mathrm{~s}, 3 \mathrm{H}), 3.69-3.60(\mathrm{~m}, 2 \mathrm{H}), 3.55(\mathrm{~s}, 1 \mathrm{H})$, $3.02(\mathrm{~d}, J=19.0 \mathrm{~Hz}, 1 \mathrm{H}), 2.71$ (dd, $J=19.2,8.5 \mathrm{~Hz}, 1 \mathrm{H}) .{ }^{13}$ C NMR (100 MHz, Chloroform-d) $\delta 213.92$, 160.07, 139.05, 137.84, 133.86, 131.06, 130.20, 127.21, 124.23, 123.45, 122.64, 121.01, 114.76, 113.16, 108.56, 106.57, 70.51, 60.99, 55.30, 41.71, 33.63. HRMS (ESI) calcd. for $\mathrm{C}_{22} \mathrm{H}_{21} \mathrm{~N}_{2} \mathrm{O}_{2}[\mathrm{M}+\mathrm{H}]: 345.1603$, found: 345.1608 .

(9aR,10S,12aR)-10-(3-fluorophenyl)-9a,10,12,12a-tetrahydrobenzo[b]cyclopenta[f]pyrrolo[1,2-d][1,4]diazepin-11(9H)-one (3fa):

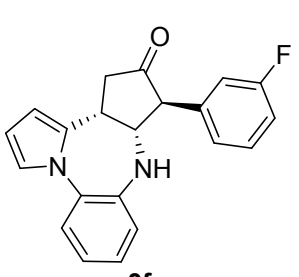

$3 f a$

White foamy solid after flash chromatography (PE:EA = 10:1), $24.5 \mathrm{mg}, 73 \%$ yield. The enantiomeric purity of the product was determined by HPLC analysis: er 93:7 (Chiralcel IA, hexane $/ i$-PrOH $=70: 30$, flow rate $1.0 \mathrm{~mL} / \mathrm{min}, \lambda=254$ $\mathrm{nm}), t_{\mathrm{r}}($ major $)=8.800 \mathrm{~min}, t_{\mathrm{r}}($ minor $)=6.596 \mathrm{~min} .{ }^{1} \mathbf{H}$ NMR (400 MHz, Chloroform- $d) \delta 7.42-7.29(\mathrm{~m}, 2 \mathrm{H}), 7.21-7.11(\mathrm{~m}, 2 \mathrm{H}), 7.07-6.97(\mathrm{~m}, 2 \mathrm{H})$, 6.97-6.90 (m, 2H), 6.90-6.83 (m, 1H), 6.35-6.26 (m, 1H), 6.22-6.10 (m, 1H), $4.38-4.23(\mathrm{~m}, 1 \mathrm{H}), 3.73-3.59(\mathrm{~m}, 2 \mathrm{H}), 3.57(\mathrm{~s}, 1 \mathrm{H}), 3.04(\mathrm{~d}, J=19.4 \mathrm{~Hz}, 1 \mathrm{H}), 2.72(\mathrm{dd}, J=19.1,8.5$ $\mathrm{Hz}, 1 \mathrm{H}) .{ }^{13} \mathrm{C}$ NMR (100 MHz, Chloroform-d) $\delta 213.27,163.15$ (d, $\left.J=246.8 \mathrm{~Hz}\right), 138.86,133.84$, $130.72(\mathrm{~d}, J=18.0 \mathrm{~Hz}) .127 .24,124.72(\mathrm{~d}, J=2.7 \mathrm{~Hz}), 124.36,123.49,122.69,121.66,115.93,115.71$, 114.87, 114.66, 108.61, 106.65, 70.28, 60.60, 41.60, 33.62. HRMS (ESI) calcd. for $\mathrm{C}_{21} \mathrm{H}_{18} \mathrm{FN}_{2} \mathrm{O}$ [M+H]: 333.1403, found: 333.1409 .

(9aR,10S,12aR)-10-(3-chlorophenyl)-9a,10,12,12a-tetrahydrobenzo[b]cyclopenta[f]pyrrolo[1,2d][1,4]diazepin-11(9H)-one (3ga):

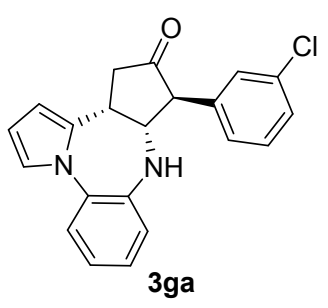

White foamy solid after flash chromatography (PE:EA = 10:1), $23.5 \mathrm{mg}$, $67 \%$ yield. The enantiomeric purity of the product was determined by HPLC analysis: er 92.5:7.5 (Chiralcel IA, hexane $/ i-\mathrm{PrOH}=70: 30$, flow rate 1.0 $\mathrm{mL} / \mathrm{min}, \lambda=54 \mathrm{~nm}), t_{\mathrm{r}}$ (major) $=8.106 \mathrm{~min}, t_{\mathrm{r}}($ minor $)=6.695 \mathrm{~min} .{ }^{1} \mathbf{H}$ NMR (400 MHz, Chloroform-d) $\delta$ 7.40-7.34 (m, 1H), 7.33-7.28 (m, 2H), 7.20-7.17 $(\mathrm{m}, 3 \mathrm{H}), 7.06-6.97(\mathrm{~m}, 2 \mathrm{H}), 6.96-6.91(\mathrm{~m}, 1 \mathrm{H}), 6.34-6.25(\mathrm{~m}, 1 \mathrm{H}), 6.18-$ $6.10(\mathrm{~m}, 1 \mathrm{H}), 4.30(\mathrm{t}, J=9.4 \mathrm{~Hz}, 1 \mathrm{H}), 3.66(\mathrm{t}, J=10.4 \mathrm{~Hz}, 2 \mathrm{H}), 3.51(\mathrm{~s}, 1 \mathrm{H}), 3.03(\mathrm{~d}, J=19.1 \mathrm{~Hz}, 1 \mathrm{H})$, 2.72 (dd, $J=19.1,8.4 \mathrm{~Hz}, 1 \mathrm{H}) .{ }^{13} \mathbf{C}$ NMR (100 MHz, Chloroform-d) $\delta$ 213.27, 138.83, 138.26, 134.95, 130.76, 130.39, 128.94, 127.98, 127.35, 124.36, 123.49, 122.69, 121.66, 108.61, 60.62, 41.63, 33.63. HRMS (ESI) calcd. for $\mathrm{C}_{21} \mathrm{H}_{18} \mathrm{ClN}_{2} \mathrm{O}[\mathrm{M}+\mathrm{H}]: 349.1108$, found: 349.1100 .

(9aR,10S,12aR)-10-(4-chlorophenyl)-9a,10,12,12a-tetrahydrobenzo[b]cyclopenta[f]pyrrolo[1,2-d][1,4] diazepin-11(9H)-one (3ha):

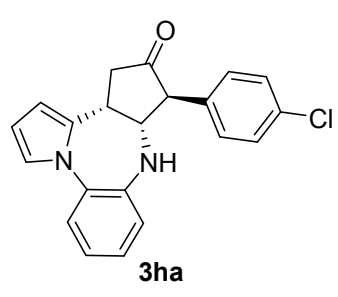

White foamy solid after flash chromatography (PE:EA = 10:1), $23.1 \mathrm{mg}, 68 \%$ yield. The enantiomeric purity of the product was determined by HPLC analysis: $\operatorname{er} 87.7: 12.3$ (Chiralcel IA, hexane $/ i$-PrOH $=70: 30$, flow rate 1.0 $\mathrm{mL} / \mathrm{min}, \lambda=360 \mathrm{~nm}), t_{\mathrm{r}}($ major $)=15.440 \mathrm{~min}, t_{\mathrm{r}}($ minor $)=6.845 \mathrm{~min} .{ }^{1} \mathbf{H}$ 
NMR (400 MHz, Chloroform-d) $\delta$ 7.44-7.29 (m, 3H), 7.22-7.12 (m, 2H), 7.12-7.04 (m, 2H), 7.03-6.96 $(\mathrm{m}, 1 \mathrm{H}), 6.96-6.88(\mathrm{~m}, 1 \mathrm{H}), 6.30(\mathrm{~s}, 1 \mathrm{H}), 6.16(\mathrm{~s}, 1 \mathrm{H}), 4.38-4.20(\mathrm{~m}, 1 \mathrm{H}), 3.81-3.57(\mathrm{~m}, 2 \mathrm{H}), 3.50(\mathrm{~s}$, 1H), $3.03(\mathrm{~d}, J=19.2 \mathrm{~Hz}, 1 \mathrm{H}), 2.71(\mathrm{dd}, J=19.1,8.1 \mathrm{~Hz}, 1 \mathrm{H}) .{ }^{13} \mathrm{C}$ NMR (100 MHz, Chloroform-d) $\delta$ 213.56, 138.85, 130.30, 127.25, 124.38, 123.50, 122.65, 121.66, 108.61, 106.66, 70.36, 60.35, 41.55, 33.61. HRMS (ESI) calcd. for $\mathrm{C}_{21} \mathrm{H}_{18} \mathrm{ClN}_{2} \mathrm{O}[\mathrm{M}+\mathrm{H}]: 349.1108$, found: 349.1105 .

(9aR,10S,12aR)-10-(naphthalen-1-yl)-9a,10,12,12a-tetrahydrobenzo[b]cyclopenta[f]pyrrolo[1,2-d][1,4] diazepin-11(9H)-one (3ia):

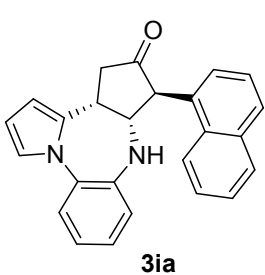

White foamy solid after flash chromatography (PE:EA = 10:1), 33.1 mg, 91\% yield The enantiomeric purity of the product was determined by HPLC analysis: er 91.5:8.5 (Chiralcel IA, hexane $/ i$-PrOH $=70: 30$, flow rate $1.0 \mathrm{~mL} / \mathrm{min}, \lambda=360 \mathrm{~nm}$ ), $t_{\mathrm{r}}($ major $)=11.424 \mathrm{~min}, t_{\mathrm{r}}$ (minor) $=8.775 \mathrm{~min} .{ }^{\mathbf{1}} \mathbf{H}$ NMR $(400 \mathrm{MHz}$, Chloroformd) $\delta 7.93-7.45(\mathrm{~m}, 3 \mathrm{H}), 7.54-7.40(\mathrm{~m}, 3 \mathrm{H}), 7.40-7.33(\mathrm{~m}, 1 \mathrm{H}), 7.29-7.22(\mathrm{~m}, 1 \mathrm{H})$, $7.11(\mathrm{p}, J=7.3 \mathrm{~Hz}, 2 \mathrm{H}), 7.02(\mathrm{~s}, 1 \mathrm{H}), 6.78(\mathrm{~d}, J=7.3 \mathrm{~Hz}, 1 \mathrm{H}), 6.34$ (s, 1H), 6.24 (s, 1H), $4.58(\mathrm{~s}, 1 \mathrm{H}), 4.40(\mathrm{~s}, 1 \mathrm{H}), 3.75(\mathrm{t}, J=7.2 \mathrm{~Hz}, 1 \mathrm{H}), 3.43(\mathrm{~s}, 1 \mathrm{H}), 3.14(\mathrm{~d}, J=19.0 \mathrm{~Hz}, 1 \mathrm{H}), 2.91$ (dd, $J=18.7,5.5 \mathrm{~Hz}, 1 \mathrm{H}) .{ }^{13}$ C NMR see: B. V. Reddy, Y. V. Reddy, K. K. Singarapu, Org. Biomol. Chem. 2016, 14, 1111-1116. HRMS (ESI) calcd. for $\mathrm{C}_{25} \mathrm{H}_{21} \mathrm{~N}_{2} \mathrm{O}$ [M+H]: 365.1654, found: 365.1659 .

(9aR,10S,12aR)-10-(naphthalen-2-yl)-9a,10,12,12a-tetrahydrobenzo[b]cyclopenta[f]pyrrolo[1,2-d][1,4] diazepin-11(9H)-one (3ja):

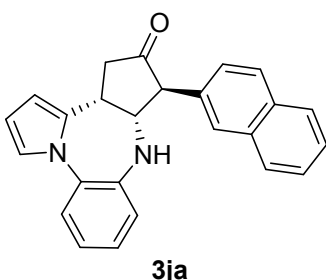

White solid after flash chromatography (PE:EA = 10:1), $33.8 \mathrm{mg}, 93 \%$ yield. The enantiomeric purity of the product was determined by HPLC analysis: er 92.5:7.5 (Chiralcel IA, hexane $/ i-\mathrm{PrOH}=70: 30$, flow rate $1.0 \mathrm{~mL} / \mathrm{min}, \lambda=360$ $\mathrm{nm}), t_{\mathrm{r}}($ major $)=12.622 \mathrm{~min}, t_{\mathrm{r}}($ minor $)=7.805 \mathrm{~min} .{ }^{1} \mathbf{H} \mathbf{~ N M R}(400 \mathrm{MHz}$, Chloroform- $\boldsymbol{d}) \delta 7.92-7.68(\mathrm{~m}, 3 \mathrm{H}), 7.66-7.54(\mathrm{~m}, 1 \mathrm{H}), 7.54-7.41(\mathrm{~m}, 2 \mathrm{H})$, $7.40-7.28(\mathrm{~m}, 1 \mathrm{H}), 7.24-7.15(\mathrm{~m}, 1 \mathrm{H}), 7.10(\mathrm{~s}, 2 \mathrm{H}), 6.98(\mathrm{~s}, 1 \mathrm{H}), 7.02-$ $6.94(\mathrm{~m} \mathrm{1H}), 6.30(\mathrm{~s}, 1 \mathrm{H}), 6.17(\mathrm{~s}, 1 \mathrm{H}), 4.46-4.24(\mathrm{~m}, 1 \mathrm{H}), 3.88-3.74(\mathrm{~m}, 1 \mathrm{H}), 3.64(\mathrm{~s}, 1 \mathrm{H}), 3.53$ (s, 1H), $3.03(\mathrm{~d}, J=19.1 \mathrm{~Hz}, 1 \mathrm{H}), 2.72(\mathrm{dd}, J=19.0,8.1 \mathrm{~Hz}, 1 \mathrm{H}) .{ }^{13} \mathbf{C}$ NMR (100 MHz, Chloroform-d) $\delta$ 139.07, 133.87, 133.74, 133.58, 132.86, 131.09, 129.06, 128.57, 127.81, 127.80, 127.24, 126.54, 126.30, 126.26, 124.24, 123.49, 122.64, 121.68, 108.65, 106.67, 70.49, 61.24, 41.80, 33.78. HRMS (ESI) calcd. for $\mathrm{C}_{25} \mathrm{H}_{21} \mathrm{~N}_{2} \mathrm{O}[\mathrm{M}+\mathrm{H}]: 365.1654$, found: 365.1656 .

9aR,10S,12aR)-10-(2,3-dimethoxyphenyl)-9a,10,12,12a-tetrahydrobenzo[b]cyclopenta[f]pyrrolo[1,2d][1,4]diazepin-11(9H)-one (3ka):

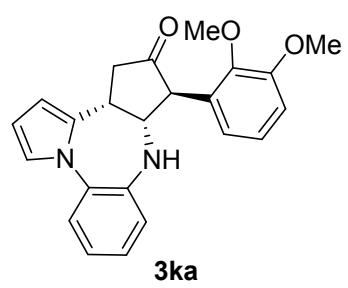

White foamy solid after flash chromatography (PE:EA = 5:1), $34.2 \mathrm{mg}$, $91 \%$ yield. The enantiomeric purity of the product was determined by HPLC analysis: $\operatorname{er} 96: 4$ (Chiralcel IA, hexane $/ i-\mathrm{PrOH}=70: 30$, flow rate $1.0 \mathrm{~mL} / \mathrm{min}$, 
$\lambda=254 \mathrm{~nm}), t_{\mathrm{r}}($ major $)=21.089 \mathrm{~min}, t_{\mathrm{r}}($ minor $)=8.750 \mathrm{~min} .{ }^{1} \mathbf{H}$ NMR (400 MHz, Chloroform- $\left.\boldsymbol{d}\right) \delta$ $7.48-7.31(\mathrm{~m}, 1 \mathrm{H}), 7.21-7.10(\mathrm{~m}, 2 \mathrm{H}), 7.05-6.97(\mathrm{~m}, 1 \mathrm{H}), 6.97-6.90(\mathrm{~m}, 1 \mathrm{H}), 6.91-6.83(\mathrm{~m}, 1 \mathrm{H})$, $6.76-6.66(\mathrm{~m}, 1 \mathrm{H}), 6.65(\mathrm{~s}, 1 \mathrm{H}), 6.31(\mathrm{~s}, 1 \mathrm{H}), 6.17(\mathrm{~s}, 1 \mathrm{H}), 4.39-4.22(\mathrm{~m}, 1 \mathrm{H}), 3.87(\mathrm{~s}, 3 \mathrm{H}), 3.84(\mathrm{~s}$, $3 \mathrm{H}), 3.72-3.59$ (m, 2H), 3.59 (s, 1H), 3.03 (d, $J=19.2 \mathrm{~Hz}, 1 \mathrm{H}), 2.72$ (dd, $J=19.1,8.2 \mathrm{~Hz}, 1 \mathrm{H}) .{ }^{13} \mathrm{C}$ NMR (100 MHz, Chloroform-d) $\delta$ 214.42, 149.28, 127.21, 124.24, 123.45, 122.62, 121.59, 120.72, 112.30, 111.78, 108.54, 106.54, 70.61, 60.68, 55.95, 41.51. HRMS (ESI) calcd. for $\mathrm{C}_{23} \mathrm{H}_{23} \mathrm{~N}_{2} \mathrm{O}_{3}[\mathrm{M}+\mathrm{H}]$ : 375.1708, found: 375.1703 .

(9aR,10S,12aR)-10-(3,5-dimethoxyphenyl)-9a,10,12,12a-tetrahydrobenzo[b]cyclopenta[f]pyrrolo[1,2d][1,4]diazepin-11(9H)-one (3la):

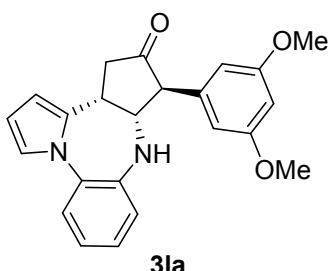

$31 a$

White foamy solid after flash chromatography (PE:EA = 10:1), 35.1 mg, 93\% yield. The enantiomeric purity of the product was determined by HPLC analysis: er 95.5:4.5 (Chiralcel IA, hexane/ $i-\mathrm{PrOH}=70: 30$, flow rate 1.0 $\mathrm{mL} / \min , \lambda=254 \mathrm{~nm}), t_{\mathrm{r}}($ major $)=11.417 \mathrm{~min}, t_{\mathrm{r}}($ minor $)=9.883 \mathrm{~min} .{ }^{1} \mathbf{H}$ NMR (400 MHz, Chloroform-d) $\delta$ 7.45-7.30 (m, 1H), $7.16(\mathrm{~s}, 2 \mathrm{H}), 6.99$ (s, $1 \mathrm{H}), 6.96-6.90(\mathrm{~m}, 1 \mathrm{H}), 6.42(\mathrm{~s}, 1 \mathrm{H}), 6.36-6.29(\mathrm{~m}, 3 \mathrm{H}), 6.16(\mathrm{~s}, 1 \mathrm{H}), 4.40-$ $4.26(\mathrm{~m}, 1 \mathrm{H}), 3.77(\mathrm{~s}, 6 \mathrm{H}), 3.69-3.51(\mathrm{~m}, 3 \mathrm{H}), 3.01(\mathrm{~d}, \mathrm{~J}=19.1 \mathrm{~Hz}, 1 \mathrm{H}), 2.71(\mathrm{dd}, \mathrm{J}=19.0,8.2 \mathrm{~Hz}$, 1H). ${ }^{13}$ C NMR (100 MHz, Chloroform-d) $\delta$ 213.74, 161.26, 139.04, 138.50, 133.84, 131.04, 123.44, 122.65, 121.60, 108.55, 106.92, 99.66, 70.44, 55.42, 33.60. HRMS (ESI) calcd. for $\mathrm{C}_{23} \mathrm{H}_{23} \mathrm{~N}_{2} \mathrm{O}_{3}[\mathrm{M}+\mathrm{H}]$ : 375.1708, found: 375.1701 .

(9aR,10S,12aR)-10-(4-fluoro-2-methylphenyl)-9a,10,12,12a-tetrahydrobenzo[b]cyclopenta[f]pyrrolo [1,2-d][1,4]diazepin-11(9H)-one (3ma):

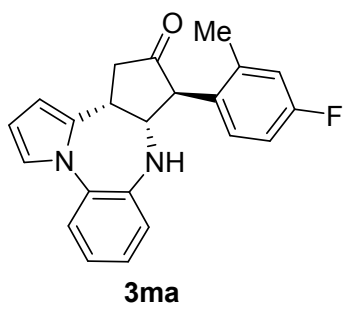

White foamy solid after flash chromatography (PE:EA 10:1), $30.2 \mathrm{mg}$, $87 \%$ yield. The enantiomeric purity of the product was determined by HPLC analysis: er 91.5:8.5 (Chiralcel IA, hexane/ $i-\mathrm{PrOH}=70: 30$, flow rate 1.0 $\mathrm{mL} / \mathrm{min}, \lambda=254 \mathrm{~nm}), t_{\mathrm{r}}$ (major) $=7.315 \mathrm{~min}, t_{\mathrm{r}}($ minor $)=5.833 \mathrm{~min} .{ }^{1} \mathbf{H}$ NMR (400 MHz, Chloroform-d) $\delta 7.41-7.31(\mathrm{~m}, 1 \mathrm{H}), 7.21-7.08(\mathrm{~m}, 2 \mathrm{H})$, 7.03-6.95 (m, 2H), 6.95-6.86 (m, 3H), 6.29 (s, 1H), 6.17 (s, 1H), 4.40 (dd, $J$ $=10.4,6.5 \mathrm{~Hz}, 1 \mathrm{H}), 3.90(\mathrm{~d}, J=10.9 \mathrm{~Hz}, 1 \mathrm{H}), 3.68(\mathrm{t}, J=7.2 \mathrm{~Hz}, 1 \mathrm{H}), 3.48$ (s, 1H), 3.02 (d, $J=19.3 \mathrm{~Hz}, 1 \mathrm{H}), 2.72(\mathrm{dd}, J=19.1,8.3 \mathrm{~Hz}, 1 \mathrm{H}), 2.24$ (s, 3H). ${ }^{13} \mathbf{C}$ NMR (100 MHz, Chloroform-d) $\delta 213.85,161.95(\mathrm{~d}, J=246.3 \mathrm{~Hz}), 139.02,133.89,130.36(\mathrm{~d}, J=2.7 \mathrm{~Hz}), 127.16,124.27$, 123.46, 122.60, 121.62, 117.82, 117.62, 113.51, 113.30, 108.55, 69.85, 41.48, 33.78, 20.30. HRMS (ESI) calcd. for $\mathrm{C}_{22} \mathrm{H}_{20} \mathrm{FN}_{2} \mathrm{O}[\mathrm{M}+\mathrm{H}]: 347.1561$, found: 347.1557 .

(9aR,10S,12aR)-10-(4-fluoro-3-methylphenyl)-9a,10,12,12a-tetrahydrobenzo[b]cyclopenta[f]pyrrolo [1,2-d][1,4]diazepin-11(9H)-one (3na):

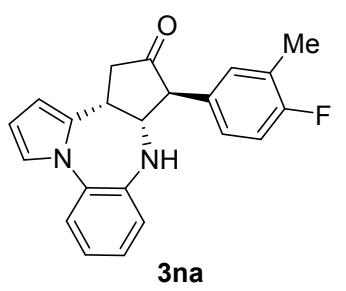

White foamy solid after flash chromatography (PE:EA = 10:1), $24.6 \mathrm{mg}, 69 \%$ yield. The enantiomeric purity of the product was determined by HPLC analysis: er 93:7 (Chiralcel IA, hexane/ $i-\mathrm{PrOH}=70: 30$, flow rate $1.0 \mathrm{~mL} / \mathrm{min}$, 
$\lambda=254 \mathrm{~nm}), t_{\mathrm{r}}($ major $)=8.895 \mathrm{~min}, t_{\mathrm{r}}($ minor $)=5.882 \mathrm{~min} .{ }^{\mathbf{1}} \mathbf{H}$ NMR (400 MHz, Chloroform- $\left.\boldsymbol{d}\right) \delta 7.44-$ $7.30(\mathrm{~m}, 1 \mathrm{H}), 7.21-7.11(\mathrm{~m}, 2 \mathrm{H}), 7.06-6.97(\mathrm{~m}, 2 \mathrm{H}), 6.97-6.88(\mathrm{~m}, 3 \mathrm{H}), 6.30(\mathrm{~s}, 1 \mathrm{H}), 6.15(\mathrm{~s}, 1 \mathrm{H}), 4.35-$ $4.17(\mathrm{~m}, 1 \mathrm{H}), 3.74-3.56(\mathrm{~m}, 2 \mathrm{H}), 3.51(\mathrm{~s}, 1 \mathrm{H}), 3.02$ (d, $J=19.2 \mathrm{~Hz}, 1 \mathrm{H}), 2.71$ (dd, $J=19.1,8.3 \mathrm{~Hz}, 1 \mathrm{H})$, 2.26 (s, 3H). ${ }^{13}$ C NMR (100 MHz, Chloroform-d) $\delta 214.18,160.94$ (d, $\left.J=245.3 \mathrm{~Hz}\right), 159.73,138.97$, 133.87, 132.07 (d, $J=5.3 \mathrm{~Hz}), 127.74,127.21,125.71$ (d, $J=17.4 \mathrm{~Hz}), 124.30,123.48,122.63,121.62$, 115.80, 115.58, 108.58, 106.60, 70.55, 60.30, 41.54, 33.58, 14.69. HRMS (ESI) calcd. for $\mathrm{C}_{22} \mathrm{H}_{20} \mathrm{FN}_{2} \mathrm{O}$ $[\mathrm{M}+\mathrm{H}]: 347.1561$, found: 347.1559 .

(9aR,10S,12aR)-10-(3,4,5-trimethoxyphenyl)-9a,10,12,12a-tetrahydrobenzo[b]cyclopenta[f]pyrrolo[1,2-d][1,4]diazepin-11(9H)-one (3oa):

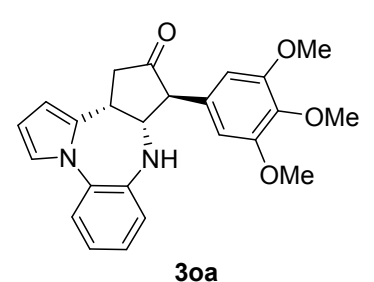

White foamy solid after flash chromatography (PE:EA = 4:1), $36.8 \mathrm{mg}, 91 \%$ yield. The enantiomeric purity of the product was determined by HPLC analysis: er 98.5:1.5 (Chiralcel IA, hexane $/ i-\mathrm{PrOH}=70: 30$, flow rate 1.0 $\mathrm{mL} / \mathrm{min}, \lambda=254 \mathrm{~nm}), t_{\mathrm{r}}$ (major) $=7.983 \mathrm{~min}, t_{\mathrm{r}}($ minor $)=13.919 \mathrm{~min} .{ }^{1} \mathbf{H}$ NMR (400 MHz, Chloroform-d) $87.44-7.33(\mathrm{~m}, 1 \mathrm{H}), 7.23-7.11(\mathrm{~m}, 2 \mathrm{H})$, $7.04-6.92(\mathrm{~m}, 2 \mathrm{H}), 6.38-6.32(\mathrm{~m}, 2 \mathrm{H}), 6.31(\mathrm{~s}, 1 \mathrm{H}), 6.17(\mathrm{~s}, 1 \mathrm{H}), 4.29(\mathrm{dd}$, $J=10.7,6.6 \mathrm{~Hz}, 1 \mathrm{H}), 3.85(\mathrm{~s}, 3 \mathrm{H}), 3.83(\mathrm{~s}, 6 \mathrm{H}), 3.72-3.60(\mathrm{~m}, 1 \mathrm{H}), 3.59(\mathrm{~s}, 1 \mathrm{H}), 3.11-2.96(\mathrm{~m}, 1 \mathrm{H})$, $2.81-2.70$ (m, 1H). ${ }^{13}$ C NMR (100 MHz, Chloroform-d) $\delta 153.72,138.96,133.81,131.79,127.23$, 124.29, 123.45, 122.66, 121.62, 106.57, 70.72, 61.44, 60.82, 56.18, 41.59, 33.53. HRMS (ESI) calcd. for $\mathrm{C}_{24} \mathrm{H}_{25} \mathrm{~N}_{2} \mathrm{O}_{4}[\mathrm{M}+\mathrm{H}]$ : 405.1814 , found: 405.1814 .

(9aR,10S,12aR)-10-(thiophen-3-yl)-9a,10,12,12a-tetrahydrobenzo[b]cyclopenta[f]pyrrolo[1,2-d][1,4] diazepin-11(9H)-one (3pa):

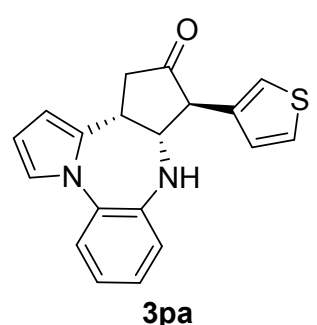

3 ра

Red foamy solid after flash chromatography (PE:EA = 10:1), $38.4 \mathrm{mg}, 89 \%$ yield. The enantiomeric purity of the product was determined by HPLC analysis: $e r$ 92.5:7.5 (Chiralcel IA, hexane $/ i-\mathrm{PrOH}=70: 30$, flow rate $1.0 \mathrm{~mL} / \mathrm{min}, \lambda=254$ $\mathrm{nm}), t_{\mathrm{r}}($ major $)=12.666 \mathrm{~min}, t_{\mathrm{r}}($ minor $)=7.241 \mathrm{~min} .{ }^{\mathbf{1}} \mathbf{H}$ NMR (400 MHz, Chloroform-d) $\delta 7.41-7.34(\mathrm{~m}, 2 \mathrm{H}), 7.21-7.15(\mathrm{~m}, 2 \mathrm{H}), 7.13(\mathrm{~s}, 1 \mathrm{H}), 7.04-$ $6.89(\mathrm{~m}, 3 \mathrm{H}), 6.30(\mathrm{~s}, 1 \mathrm{H}), 6.16(\mathrm{~s}, 1 \mathrm{H}), 4.36-4.20(\mathrm{~m}, 1 \mathrm{H}), 3.80(\mathrm{~d}, J=11.1$ $\mathrm{Hz}, 1 \mathrm{H}), 3.65$ (t, $J=7.3 \mathrm{~Hz}, 1 \mathrm{H}), 3.60$ (s, 1H), 3.00 (d, $J=18.9 \mathrm{~Hz}, 1 \mathrm{H}), 2.71$ $(\mathrm{dd}, J=19.1,8.3 \mathrm{~Hz} 1 \mathrm{H}) .{ }^{13} \mathbf{C}$ NMR (100 MHz, Chloroform-d) $\delta 213.31,127.21,126.92,123.45,123.29$, 122.68, 108.58, 76.76, 69.90, 56.15, 41.14. HRMS (ESI) calcd. for $\mathrm{C}_{19} \mathrm{H}_{17} \mathrm{~N}_{2} \mathrm{OS}[\mathrm{M}+\mathrm{H}]: 321.1062$, found: 321.1065 .

(4R,5S)-4-((2-(1H-indol-1-yl)phenyl)amino)-5-(3,4,5-trimethoxyphenyl)cyclopent-2-en-1-one (3ob):

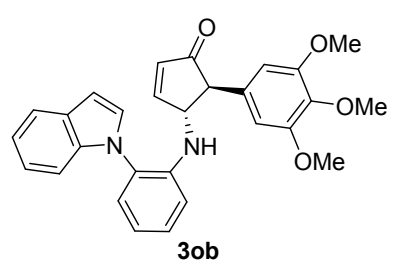
Yellow foamy solid after flash chromatography (PE:EA =4:1), $36.8 \mathrm{mg}$, $91 \%$ yield. The enantiomeric purity of the product was determined by HPLC analysis: $97 \%$ ee (Chiralcel IA, hexane $/ i-\mathrm{PrOH}=70: 30$, flow rate $1.0 \mathrm{~mL} / \mathrm{min}, \lambda=254 \mathrm{~nm}), t_{\mathrm{r}}$ (major) $=24.098 \mathrm{~min}, t_{\mathrm{r}}($ minor $)=25.164$ min. ${ }^{1}$ H NMR (400 MHz, Chloroform-d) $\delta 7.70(\mathrm{dd}, J=6.0,2.9 \mathrm{~Hz}$, 
1H), $7.64-7.50(\mathrm{~m}, 1 \mathrm{H}), 7.25-7.05(\mathrm{~m}, 6 \mathrm{H}), 6.85(\mathrm{t}, J=7.5 \mathrm{~Hz}, 1 \mathrm{H}), 6.76-6.57(\mathrm{~m}, 2 \mathrm{H}), 6.28(\mathrm{dd}, J$ $=23.8,6.8 \mathrm{~Hz}, 3 \mathrm{H}), 4.69(\mathrm{~s}, 1 \mathrm{H}), 3.84(\mathrm{~s}, 3 \mathrm{H}), 3.80(\mathrm{~s}, 6 \mathrm{H}), 3.16(\mathrm{~d}, J=26.7 \mathrm{~Hz}, 1 \mathrm{H}) .{ }^{13} \mathbf{C} \mathbf{N M R}(\mathbf{1 0 0}$ MHz, Chloroform-d) $\delta$ 205.79, 161.22, 161.09, 153.62, 142,69, 142.55, 137.42, 136.40, 135.03, $129.407,128.94,128.65,128.55,125.38,122.48,122.42,112.97,112.78,110.55,105.08,103.83,63.15$, 63.01, 60.85, 60.57, 60.47, 56.14. HRMS (ESI) calcd. for $\mathrm{C}_{28} \mathrm{H}_{27} \mathrm{~N}_{2} \mathrm{O}_{4}[\mathrm{M}+\mathrm{H}]$ : 455.1970, found: 455.1970 .

(9aR,10S,12aR)-8-methyl-10-(3,4,5-trimethoxyphenyl)-9a,10,12,12a-tetrahydrobenzo[b]cyclopenta[f] pyrrolo[1,2-d][1,4]diazepin-11(9H)-one (4oe):

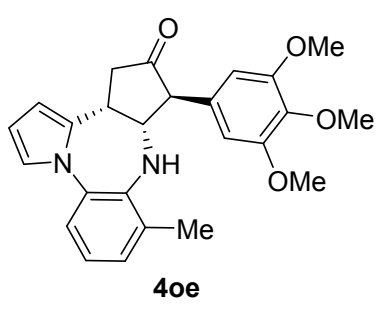

Yellow foamy solid after flash chromatography (PE:EA = 3:1), $31.6 \mathrm{mg}$, $75 \%$ yield. The enantiomeric purity of the product was determined by HPLC analysis: er 93.5:6.5 (Chiralcel IA, hexane $/ i$-PrOH $=70: 30$, flow rate $1.0 \mathrm{~mL} / \mathrm{min}, \lambda=254 \mathrm{~nm}), t_{\mathrm{r}}$ (major) $=8.002 \mathrm{~min}, t_{\mathrm{r}}($ minor $)=13.209$ min. ${ }^{1}$ H NMR (400 MHz, Chloroform-d) $\delta$ 7.19-7.01 (m, 2H), 6.95-6.88 $(\mathrm{m}, 1 \mathrm{H}), 6.87-6.76(\mathrm{~m}, 1 \mathrm{H}), 6.31(\mathrm{~s}, 3 \mathrm{H}), 6.12(\mathrm{~s}, 1 \mathrm{H}), 4.24-4.09(\mathrm{~m}, 1 \mathrm{H})$, $3.84(\mathrm{~s}, 3 \mathrm{H}), 3.83(\mathrm{~s}, 6 \mathrm{H}), 3.68-3.58(\mathrm{~m}, 1 \mathrm{H}), 3.53(\mathrm{~d}, J=10.9 \mathrm{~Hz}, 1 \mathrm{H}), 3.48(\mathrm{~s}, 1 \mathrm{H}), 3.04(\mathrm{~d}, J=19.3$ Hz, 1H), 2.75 (dd, $J=19.2,8.4$ Hz, 1H), 2.41 (s, 3H). ${ }^{13}$ C NMR (100 MHz, Chloroform-d) $\delta 214.67$, 153.68, 140.46, 132.98, 132.86, 131.94, 131.90, 127.29, 127.23, 122.94, 120.71, 107.59, 105.74, 104.72, 70.96, 61.10, 60.85, 56.17, 41.35, 33.30, 19.30. HRMS (ESI) calcd. for $\mathrm{C}_{25} \mathrm{H}_{27} \mathrm{~N}_{2} \mathrm{O}_{4}[\mathrm{M}+\mathrm{H}]:$ : 419.1971, found: 419.1976 .

(9aR,10S,12aR)-7-methyl-10-(3,4,5-trimethoxyphenyl)-9a,10,12,12a-tetrahydrobenzo[b]cyclopenta[f] pyrrolo[1,2-d][1,4]diazepin-11(9H)-one (4of):

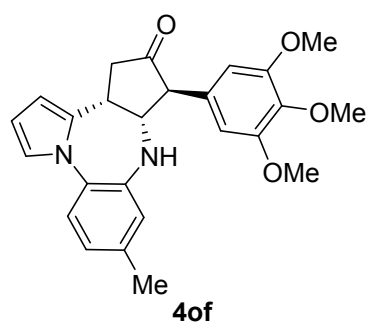

Yellow foamy solid after flash chromatography (PE:EA = 3:1), $36.8 \mathrm{mg}, 88 \%$ yield. The enantiomeric purity of the product was determined by HPLC analysis: er 97:3 (Chiralcel IA, hexane $/ i-\mathrm{PrOH}=70: 30$, flow rate 1.0 $\mathrm{mL} / \mathrm{min}, \lambda=254 \mathrm{~nm}), t_{\mathrm{r}}($ major $)=7.281 \mathrm{~min}, t_{\mathrm{r}}($ minor $)=14.291 \mathrm{~min} .{ }^{1} \mathbf{H}$ NMR (400 MHz, Chloroform-d) $\delta 7.26(\mathrm{~s}, 1 \mathrm{H}), 7.09-6.92(\mathrm{~m}, 2 \mathrm{H}), 6.82$ $(\mathrm{s}, 1 \mathrm{H}), 6.36-6.31(\mathrm{~m}, 2 \mathrm{H}), 6.30(\mathrm{~s}, 1 \mathrm{H}), 6.16(\mathrm{~s}, 1 \mathrm{H}), 4.26(\mathrm{t}, J=8.4 \mathrm{~Hz}$, $1 \mathrm{H}), 3.85(\mathrm{~s}, 3 \mathrm{H}), 3.83(\mathrm{~s}, 6 \mathrm{H}), 3.67(\mathrm{t}, J=7.4 \mathrm{~Hz}, 1 \mathrm{H}), 3.60(\mathrm{~d}, J=11.2$ Hz, 1H), 3.52 (s, 1H), 3.05 (d, $J=18.6 \mathrm{~Hz}, 1 \mathrm{H}), 2.75$ (dd, $J=19.5,8.9 \mathrm{~Hz}, 1 \mathrm{H}), 2.32(\mathrm{~s}, 3 \mathrm{H}) .{ }^{13} \mathbf{C}$ NMR (100 MHz, Chloroform-d) $\delta 214.36,153.68,138.67,137.27,131.83,131.27,130.92,124.90,123.23$, 123.17, 121.52, 108.30, 106.32, 105.68, 70.74, 61.46, 60.83, 56.15, 41.57, 33.55, 20.93. HRMS (ESI) calcd. for $\mathrm{C}_{25} \mathrm{H}_{27} \mathrm{~N}_{2} \mathrm{O}_{4}[\mathrm{M}+\mathrm{H}]: 419.1971$, found: 419.1974 .

(9aR,10S,12aR)-6-methoxy-10-(3,4,5-trimethoxyphenyl)-9a,10,12,12a-tetrahydrobenzo[b]cyclopenta [f]pyrrolo[1,2-d][1,4]diazepin-11(9H)-one (4og):

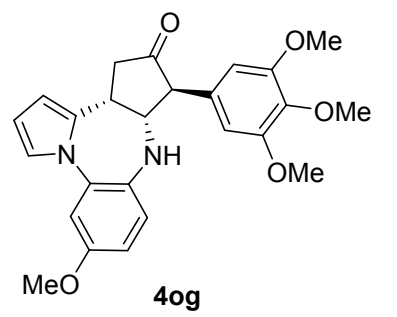

Yellow foamy solid after flash chromatography (PE:EA = 3:1), $39.3 \mathrm{mg}$, $90 \%$ yield. The enantiomeric purity of the product was determined by HPLC analysis: er 98.5:1.5 (Chiralcel IA, hexane $/ i$-PrOH $=70: 30$, flow rate 
$1.0 \mathrm{~mL} / \mathrm{min}, \lambda=254 \mathrm{~nm}), t_{\mathrm{r}}$ (major) $=9.513 \mathrm{~min}, t_{\mathrm{r}}$ (minor) $=19.406 \mathrm{~min} .{ }^{\mathbf{1}} \mathbf{H} \mathbf{~ N M R}(\mathbf{4 0 0} \mathbf{M H z}$, Chloroform- $d) \delta 7.01(\mathrm{~s}, 1 \mathrm{H}), 6.97-6.88(\mathrm{~m}, 2 \mathrm{H}), 6.79-6.66(\mathrm{~m}, 1 \mathrm{H}), 6.39-6.27(\mathrm{~m}, 3 \mathrm{H}), 6.16(\mathrm{~s}, 1 \mathrm{H})$, 4.28-4.16 (m, 1H), 3.87-3.79 (m, 12H), $3.71-3.50$ (m, 2H), 3.13-2.93 (m, 1H), 2.75 (dd, $J=19.3,8.6$ Hz, 1H). ${ }^{13}$ C NMR (100 MHz, Chloroform-d) $\delta$ 214.44, 156.71, 153.70, 134.92, 132.12, 131.90, 131.34, 123.56, 121.39, 112.07, 109.46, 108.73, 106.69, 105.76, 70.80, 61.51, 60.85, 56.17, 55.76, 41.51, 33.74. HRMS (ESI) calcd. for $\mathrm{C}_{25} \mathrm{H}_{27} \mathrm{~N}_{2} \mathrm{O}_{5}[\mathrm{M}+\mathrm{H}]$ : 435.1920, found: 435.1922 .

(9aR,10S,12aR)-6-chloro-10-(3,4,5-trimethoxyphenyl)-9a,10,12,12a-tetrahydrobenzo[b]cyclopenta[f] pyrrolo[1,2-d][1,4]diazepin-11(9H)-one (4oh):

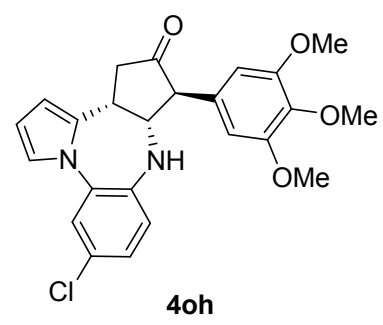

Yellow foamy solid after flash chromatography (PE:EA = 3:1), $43.2 \mathrm{mg}$, $98 \%$ yield. The enantiomeric purity of the product was determined by HPLC analysis: er 97.5:2.5 (Chiralcel IA, hexane $/ i$-PrOH $=70: 30$, flow rate $1.0 \mathrm{~mL} / \mathrm{min}, \lambda=254 \mathrm{~nm}), t_{\mathrm{r}}($ major $)=8.104 \mathrm{~min}, t_{\mathrm{r}}($ minor $)=14.083$ min. ${ }^{1}$ H NMR (400 MHz, Chloroform-d) $\delta 7.36$ (s, 1H), 7.13 (d, $J=7.8$ $\mathrm{Hz}, 1 \mathrm{H}), 7.04-6.84$ (m, 2H), 6.40-6.25 (m, 3H), 6.17 (s, 1H), 4.36-4.11 $(\mathrm{m}, 1 \mathrm{H}), 3.94-3.72(\mathrm{~m}, 9 \mathrm{H}), 3.64(\mathrm{~s}, 2 \mathrm{H}), 3.57(\mathrm{~d}, J=10.9 \mathrm{~Hz}, 1 \mathrm{H}), 3.03$ (d, $J=19.2 \mathrm{~Hz}, 1 \mathrm{H}), 2.76$ (dd, $J=19.1,8.1 \mathrm{~Hz}, 1 \mathrm{H}) .{ }^{13}$ C NMR (100 MHz, Chloroform-d) $\delta 213.86$, $153.69,137.65,134.61,131.63,130.97,128.93,127.00,123.63,123.45,121.62,109.08,107.15,105.76$, 70.50, 61.39, 60.82, 56.15, 41.59, 33.44. HRMS (ESI) calcd. for $\mathrm{C}_{24} \mathrm{H}_{24} \mathrm{ClN}_{2} \mathrm{O}_{4}[\mathrm{M}+\mathrm{H}]:$ 439.1425, found: 439.1424 .

(9aR,10S,12aR)-6-bromo-10-(3,4,5-trimethoxyphenyl)-9a,10,12,12a-tetrahydrobenzo[b]cyclopenta[f]

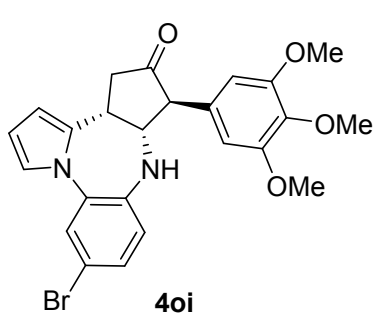
pyrrolo[1,2-d][1,4]diazepin-11(9H)-one (4oi):

Yellow foamy solid after flash chromatography (PE:EA = 3:1), $44.5 \mathrm{mg}$, $92 \%$ yield. The enantiomeric purity of the product was determined by HPLC analysis: er 97:3 (Chiralcel IA, hexane/ $i-\mathrm{PrOH}=70: 30$, flow rate $1.0 \mathrm{~mL} / \mathrm{min}, \lambda=360 \mathrm{~nm}), t_{\mathrm{r}}$ (major) $=7.949 \mathrm{~min}, t_{\mathrm{r}}($ minor $)=15.413 \mathrm{~min}$. ${ }^{1}$ H NMR (400 MHz, Chloroform-d) $\delta$ 7.32-7.20 (m, 2H), 7.17 (s, 1H), $6.95(\mathrm{~s}, 1 \mathrm{H}), 7.03-6.87(\mathrm{~m}, 3 \mathrm{H}), 6.17(\mathrm{~s}, 1 \mathrm{H}), 4.37-4.20(\mathrm{~m}, 1 \mathrm{H}), 3.90-3.75$ (m, 9H), 3.75-3.62 (m, 2H), 3.56 (d, $J=10.9 \mathrm{~Hz}, 1 \mathrm{H}), 3.03$ (d, $J=19.2 \mathrm{~Hz}, 1 \mathrm{H}), 2.76$ (dd, $J=19.2,8.1$ $\mathrm{Hz}, 1 \mathrm{H}) .{ }^{13} \mathbf{C}$ NMR (100 MHz, Chloroform-d) $\delta$ 213.77, 153.66, 132.80, 131.51, 130.85, 127.00, 125.50, 119.81, 108.91, 106.99, 105.69, 70.56, 61.31, 60.82, 56.12, 41.60, 33.39. HRMS (ESI) calcd. for $\mathrm{C}_{24} \mathrm{H}_{24} \mathrm{BrN}_{2} \mathrm{O}_{4}[\mathrm{M}+\mathrm{H}]: 483.0919$, found: 483.0923 .

(9aR,10S,12aR)-6-(trifluoromethyl)-10-(3,4,5-trimethoxyphenyl)-9a,10,12,12a-tetrahydrobenzo[b] cyclopenta[f]pyrrolo[1,2-d][1,4]diazepin-11(9H)-one (4oj):

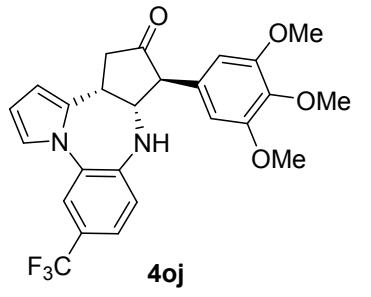

Pale foamy solid after flash chromatography (PE:EA = 3:1), $43.1 \mathrm{mg}, 91 \%$ yield. The enantiomeric purity of the product was determined by HPLC analysis: er 98:2 (Chiralcel IA, hexane $/ i-\mathrm{PrOH}=70: 30$, flow rate 1.0 $\mathrm{mL} / \mathrm{min}, \lambda=254 \mathrm{~nm}), t_{\mathrm{r}}($ major $)=5.892 \mathrm{~min}, t_{\mathrm{r}}($ minor $)=10.063 \mathrm{~min} .{ }^{1} \mathbf{H}$ NMR (400 MHz, Chloroform-d) $\delta 7.52-7.37$ (m, 2H), $7.31(\mathrm{~s}, 1 \mathrm{H}), 7.01$ 
(s, 1H), $6.32(\mathrm{~d}, J=16.1 \mathrm{~Hz}, 3 \mathrm{H}), 6.21(\mathrm{~s}, 1 \mathrm{H}), 4.34(\mathrm{~s}, 1 \mathrm{H}), 3.92(\mathrm{~s}, 1 \mathrm{H}), 3.81(\mathrm{~s}, 9 \mathrm{H}), 3.68(\mathrm{~s}, 1 \mathrm{H})$, $3.59(\mathrm{~d}, J=11.0 \mathrm{~Hz}, 1 \mathrm{H}), 3.05(\mathrm{~d}, J=19.2 \mathrm{~Hz}, 1 \mathrm{H}), 2.78(\mathrm{dd}, J=19.1,8.1 \mathrm{~Hz}, 1 \mathrm{H}) .{ }^{13} \mathbf{C}$ NMR $(100$ MHz, Chloroform-d) $\delta 213.69,153.66,139.45,137.41,136.46,131.45,131.04,129.12$ (q, $J=32.7$ $\mathrm{Hz}), 125.11,123.66,122.41,121.78,120.87(\mathrm{q}, J=3.8 \mathrm{~Hz}), 119.70(\mathrm{q}, J=4.2 \mathrm{~Hz}), 109.33,107.46$, 105.67, 70.50, 61.39, 60.76, 56.06, 41.65, 33.38. HRMS (ESI) calcd. for $\mathrm{C}_{25} \mathrm{H}_{24} \mathrm{~F}_{3} \mathrm{~N}_{2} \mathrm{O}_{4}[\mathrm{M}+\mathrm{H}]$ : 473.1688, found: 473.1681 .

(9aR,10S,12aR)-6-chloro-7-methyl-10-(3,4,5-trimethoxyphenyl)-9a,10,12,12a-tetrahydrobenzo[b] cyclopenta[f]pyrrolo[1,2-d][1,4]diazepin-11(9H)-one (4oc):

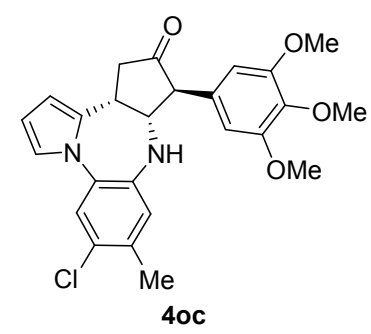

Yellow foamy solid after flash chromatography (PE:EA = 3:1), $43.8 \mathrm{mg}$, $97 \%$ yield. The enantiomeric purity of the product was determined by HPLC analysis: $95 \%$ ee (Chiralcel IA, hexane $/ i-\mathrm{PrOH}=70: 30$, flow rate $1.0 \mathrm{~mL} / \mathrm{min}, \lambda=360 \mathrm{~nm}), t_{\mathrm{r}}$ (major) $=7.448 \mathrm{~min}, t_{\mathrm{r}}($ minor $)=15.746 \mathrm{~min}$.

${ }^{1}$ H NMR (400 MHz, Chloroform- $d$ ) $\delta 7.37$ (s, 1H), 6.97 (s, 1H), 6.87 (s, $1 \mathrm{H}), 6.34-6.28(\mathrm{~m}, 3 \mathrm{H}), 6.17(\mathrm{~s}, 1 \mathrm{H}), 4.29-4.16(\mathrm{~m}, 1 \mathrm{H}), 3.84(\mathrm{~s}, 3 \mathrm{H}), 3.83$ (s, 6H), $3.73(\mathrm{~d}, \mathrm{~J}=6.6 \mathrm{~Hz}, 1 \mathrm{H}), 3.64(\mathrm{t}, \mathrm{J}=7.0 \mathrm{~Hz}, 1 \mathrm{H}), 3.57(\mathrm{~d}, \mathrm{~J}=11.1$ Hz, 1H), 3.54 (s, 1H), 3.04 (d, J = 19.3 Hz, 1H), 2.76 (dd, J = 19.4, 8.4 Hz, 1H), 2.33 (s, 3H). ${ }^{13} \mathbf{C}$ NMR (100 MHz, Chloroform-d) $\delta$ 213.96, 153.73, 137.39, 134.86, 132.35, 131.65, 130.89, 128.95, 124.60, $123.60,121.53,108.79,106.89,105.71,70.58,61.38,60.83,56.17,41.57,33.48,19.57$. HRMS (ESI) calcd. for $\mathrm{C}_{25} \mathrm{H}_{26} \mathrm{ClN}_{2} \mathrm{O}_{4}[\mathrm{M}+\mathrm{H}]: 453.1581$, found: 453.1587 .

(9aR,10S,12aR)-6,7-dimethyl-10-(3,4,5-trimethoxyphenyl)-9a,10,12,12a-tetrahydrobenzo[b]cyclopenta [f]pyrrolo[1,2-d][1,4]diazepin-11(9H)-one (4ok):

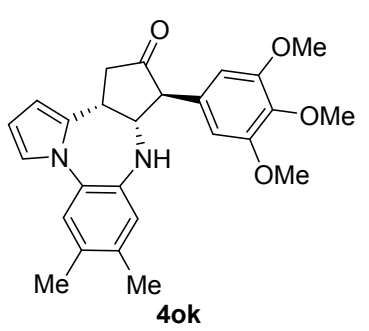

Yellow foamy solid after flash chromatography (PE:EA = 3:1), $41.1 \mathrm{mg}, 95 \%$ yield. The enantiomeric purity of the product was determined by HPLC analysis: er 98.5:1.5 (Chiralcel IA, hexane $/ i-\mathrm{PrOH}=70: 30$, flow rate 1.0 $\mathrm{mL} / \mathrm{min}, \lambda=254 \mathrm{~nm}), t_{\mathrm{r}}$ (major) $=7.224 \mathrm{~min}, t_{\mathrm{r}}($ minor $)=9.610 \mathrm{~min} .{ }^{1} \mathbf{H}$ NMR (400 MHz, Chloroform-d) $\delta 7.15$ (s, 1H), 6.99 (s, 1H), 6.78 (s, 1H), $6.31(\mathrm{~d}, J=12.6 \mathrm{~Hz}, 3 \mathrm{H}), 6.15$ (s, 1H), $4.32-4.15(\mathrm{~m}, 1 \mathrm{H}), 3.83$ (s, 9H), $3.69-3.55(\mathrm{~m}, 2 \mathrm{H}), 3.46(\mathrm{~s}, 1 \mathrm{H}), 3.04(\mathrm{~d}, J=19.2 \mathrm{~Hz}, 1 \mathrm{H}), 2.74$ (dd, $J=$ 19.2, 8.2 Hz, 1H), 2.27 (s, 3H), 2.22 (s, 3H). ${ }^{13}$ C NMR (100 MHz, Chloroform-d) $\delta$ 214.54, 153.68, $136.37,135.65,132.59,131.92,131.03,124.35,123.82,121.41,108.22,106.28,105.71,70.85,61.52$, 60.84, 56.16, 41.58, 33.67, 19.32, 19.26. HRMS (ESI) calcd. for $\mathrm{C}_{26} \mathrm{H}_{29} \mathrm{~N}_{2} \mathrm{O}_{4}[\mathrm{M}+\mathrm{H}]: 433.2127$, found: 433.2129 .

(4R,5S)-4-((2-(1H-pyrrol-1-yl)-4-(trifluoromethyl)phenyl)amino)-5-(3,4,5-trimethoxyphenyl)cyclopent-2-en-1-one (5):

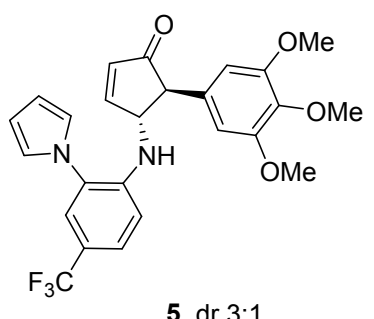

5, dr 3:1

Pale foamy solid after flash chromatography (PE:EA = 3:1), $14.2 \mathrm{mg}, 30 \%$ yield. The enantiomeric purity of the product was determined by HPLC analysis: er 97.5:2.5 (Chiralcel OD-H, hexane/ $i-\mathrm{PrOH}=80: 20$, flow rate 1.0 $\mathrm{mL} / \mathrm{min}, \lambda=210 \mathrm{~nm}), t_{\mathrm{r}}($ major $)=11.030 \mathrm{~min}, t_{\mathrm{r}}($ minor $)=9.102 \mathrm{~min} .{ }^{1} \mathbf{H}$ 
NMR (400 MHz, Chloroform-d) $\delta 7.64(\mathrm{dd}, J=5.7,2.3 \mathrm{~Hz}, 1 \mathrm{H}), 7.23(\mathrm{~d}, J=8.0 \mathrm{~Hz}, 1 \mathrm{H}), 7.10-6.89$ $(\mathrm{m}, 2 \mathrm{H}), 6.79(\mathrm{t}, J=2.1 \mathrm{~Hz}, 2 \mathrm{H}), 6.70(\mathrm{~d}, J=1.9 \mathrm{~Hz}, 1 \mathrm{H}), 6.42(\mathrm{dd}, J=5.8,1.8 \mathrm{~Hz}, 1 \mathrm{H}), 6.38(\mathrm{t}, J=2.1$ $\mathrm{Hz}, 2 \mathrm{H}), 6.32$ (t, $J=2.1 \mathrm{~Hz}, 1 \mathrm{H}), 6.29(\mathrm{~s}, 2 \mathrm{H}), 6.21(\mathrm{t}, J=2.1 \mathrm{~Hz}, 1 \mathrm{H}), 5.97(\mathrm{~s}, 1 \mathrm{H}), 4.71(\mathrm{dq}, J=7.3$, $2.2 \mathrm{~Hz}, 1 \mathrm{H}), 4.37(\mathrm{~d}, J=7.8 \mathrm{~Hz}, 1 \mathrm{H}), 3.84(\mathrm{~s}, 3 \mathrm{H}), 3.80(\mathrm{~s}, 6 \mathrm{H}), 3.78(\mathrm{~s}, 1 \mathrm{H}), 3.62(\mathrm{~s}, 2 \mathrm{H}), 3.20(\mathrm{~d}, J=$ $2.6 \mathrm{~Hz}, 1 \mathrm{H}) .{ }^{13} \mathrm{C}$ NMR (100 MHz, Chloroform-d) $\delta$ 204.16, 160.56, 158.82, 152.54, 151.97, 140.75, 136.45, 135.19, 134.42, 131.84, 128.97, 126.30, 126.18, 120.12, 119.78, 113.59, 113.55, 109.32, 108.87, $108.21,108.17,106.72,105.02,103.75,61.92,59.65,59.48,59.40,54.98,54.80,54.47$. HRMS (ESI) calcd. for C25H24F3N2O4 [M+H]: 473.1688, found: 473.1685.

(9aR,10S,11S,12aR)-6-(trifluoromethyl)-10-(3,4,5-trimethoxyphenyl)-9,9a,10,11,12,12a hexahydrobenzo[b]cyclopenta[f]pyrrolo[1,2-d][1,4]diazepin-11-ol (6):

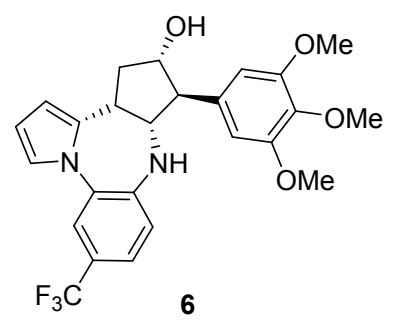

White solid after flash chromatography (PE:EA = 3:1), $54.2 \mathrm{mg}, 78 \%$ yield. The enantiomeric purity of the product was determined by HPLC analysis: er 96.5:3.5 (Chiralcel IA, hexane/ $i-\mathrm{PrOH}=60: 40$, flow rate 1.0 $\mathrm{mL} / \mathrm{min}, \lambda=254 \mathrm{~nm}), t_{\mathrm{r}}($ major $)=6.326 \mathrm{~min}, t_{\mathrm{r}}($ minor $)=8.752 \mathrm{~min} .{ }^{1} \mathbf{H}$ NMR (400 MHz, Chloroform-d) $\delta 7.46(\mathrm{~d}, J=8.3 \mathrm{~Hz}, 1 \mathrm{H}), 7.38$ (dd, $J$ $=8.2,1.9 \mathrm{~Hz}, 1 \mathrm{H}), 7.16(\mathrm{~d}, J=1.9 \mathrm{~Hz}, 1 \mathrm{H}), 6.92(\mathrm{t}, J=2.4 \mathrm{~Hz}, 1 \mathrm{H}), 6.48$ (s, 2H), $6.34(\mathrm{~m}, 2 \mathrm{H}), 4.32(\mathrm{q}, J=8.0 \mathrm{~Hz}, 1 \mathrm{H}), 4.03-3.92(\mathrm{~m}, 1 \mathrm{H}), 3.85$ $(\mathrm{s}, 6 \mathrm{H}), 3.83(\mathrm{~s}, 3 \mathrm{H}), 3.46(\mathrm{~m}, 2 \mathrm{H}), 3.01(\mathrm{t}, J=9.9 \mathrm{~Hz}, 1 \mathrm{H}), 2.57(\mathrm{~m}, 1 \mathrm{H}), 2.18(\mathrm{~m}, 2 \mathrm{H}) .{ }^{13}$ C NMR (100 MHz, Chloroform-d) $\delta 153.73,140.21,137.20,134.64,133.72,128.60$ (q, $J=25 \mathrm{~Hz}$ ), 121.96 (q, $J=$ $2.7 \mathrm{~Hz}$ ), 120.00 (q, $J=4.6 \mathrm{~Hz}), 109.22,108.60,104.60,76.35,70.73,60.78,59.72,56.10,36.58,36.50$.

HRMS (ESI) calcd. for $\mathrm{C}_{25} \mathrm{H}_{26} \mathrm{~F}_{3} \mathrm{~N}_{2} \mathrm{O}_{4}[\mathrm{M}+\mathrm{H}]$ : 475.1845, found: 475.1849 .

(9aR,10S,11S)-11-((3,5-bis(trifluoromethyl)benzyl)oxy)-6-(trifluoromethyl)-10-(3,4,5-trimethoxyphenyl)-9,9a,10,11,12,12a-hexahydrobenzo[b]cyclopenta[f]pyrrolo[1,2-d][1,4]diazepine (7):

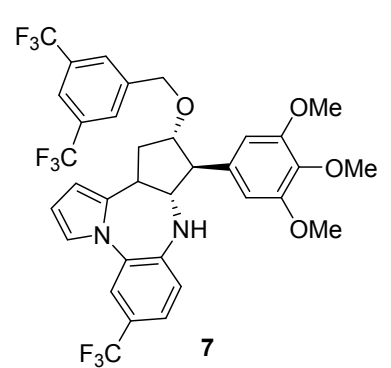

White solid after flash chromatography (PE:EA = 5:1), $73.9 \mathrm{mg}, 86 \%$ yield. The enantiomeric purity of the product was determined by HPLC analysis: er 97.5:2.5 (Chiralcel IA, hexane/ $i-\mathrm{PrOH}=70: 30$, flow rate 1.0 $\mathrm{mL} / \mathrm{min}, \lambda=254 \mathrm{~nm}), t_{\mathrm{r}}($ major $)=4.290 \mathrm{~min}, t_{\mathrm{r}}($ minor $)=4.712 \mathrm{~min} .{ }^{1} \mathbf{H}$ NMR (400 MHz, Chloroform-d) $\delta 7.46$ (d, $J=8.2 \mathrm{~Hz}, 1 \mathrm{H}), 7.38$ (d, $J=$ $8.2 \mathrm{~Hz}, 1 \mathrm{H}), 7.16$ (s, 1H), 6.92 (s, 1H), 6.48 (s, 2H), 6.33 (dt, $J=7.4,4.1$ $\mathrm{Hz}, 2 \mathrm{H}), 4.32$ (q, $J=7.9 \mathrm{~Hz}, 1 \mathrm{H}), 4.01-3.92(\mathrm{~m}, 1 \mathrm{H}), 3.85(\mathrm{~s}, 6 \mathrm{H}), 3.83$ (s, 3H), 3.46 (q, $J=7.8 \mathrm{~Hz}, 2 \mathrm{H}), 3.01(\mathrm{t}, J=9.9 \mathrm{~Hz}, 1 \mathrm{H}), 2.57(\mathrm{~m}, 1 \mathrm{H}), 2.17(\mathrm{~m}, 2 \mathrm{H}) .{ }^{13} \mathbf{C} \mathbf{N M R}(\mathbf{1 0 0}$ MHz, Chloroform-d) $\delta 153.80,141.15,140.09,137.27,136.94,135.55,133.19,132.66,131.73,131.57$ $(\mathrm{q}, J=33.1 \mathrm{~Hz}), 128.65(\mathrm{q}, J=32.4 \mathrm{~Hz}), 127.05,123.37,121.75(\mathrm{q}, J=4.5 \mathrm{~Hz}), 119.65(\mathrm{q}, J=4.4 \mathrm{~Hz})$, 109.13, 108.50, 104.36, 83.94, 71.55, 70.02, 60.80, 58.29, 56.10, 37.10, 34.06. HRMS (ESI) calcd. for $\mathrm{C}_{34} \mathrm{H}_{30} \mathrm{~F}_{9} \mathrm{~N}_{2} \mathrm{O}_{4}[\mathrm{M}+\mathrm{H}]:$ 701.2062, found: 701.2068. 
$4{ }^{1} \mathrm{H}$ and ${ }^{13} \mathrm{C}$ NMR spectra for all compounds 
₹

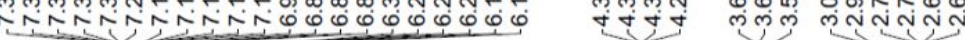
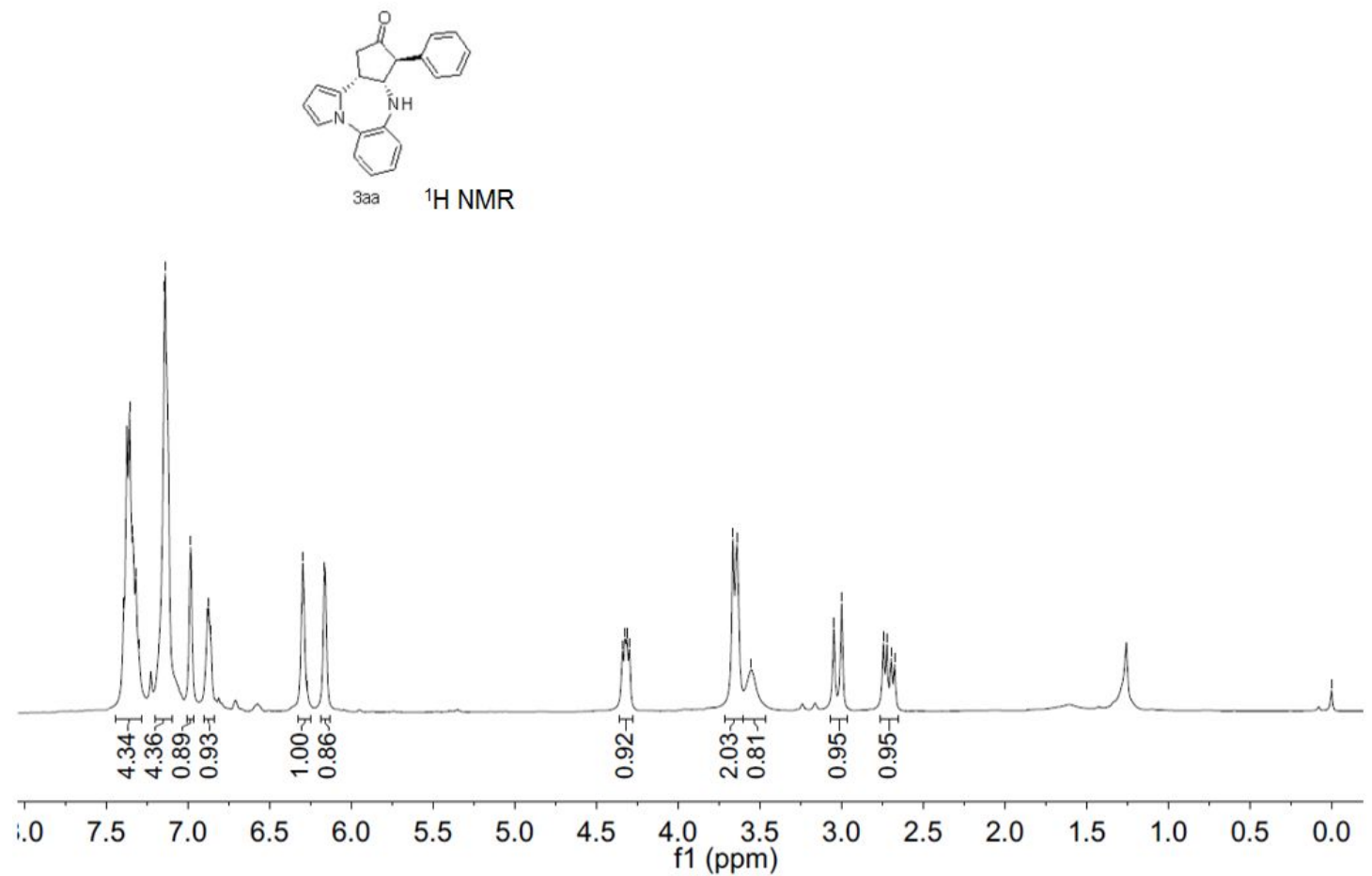


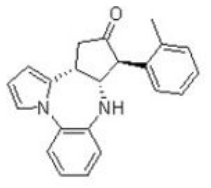

3ba

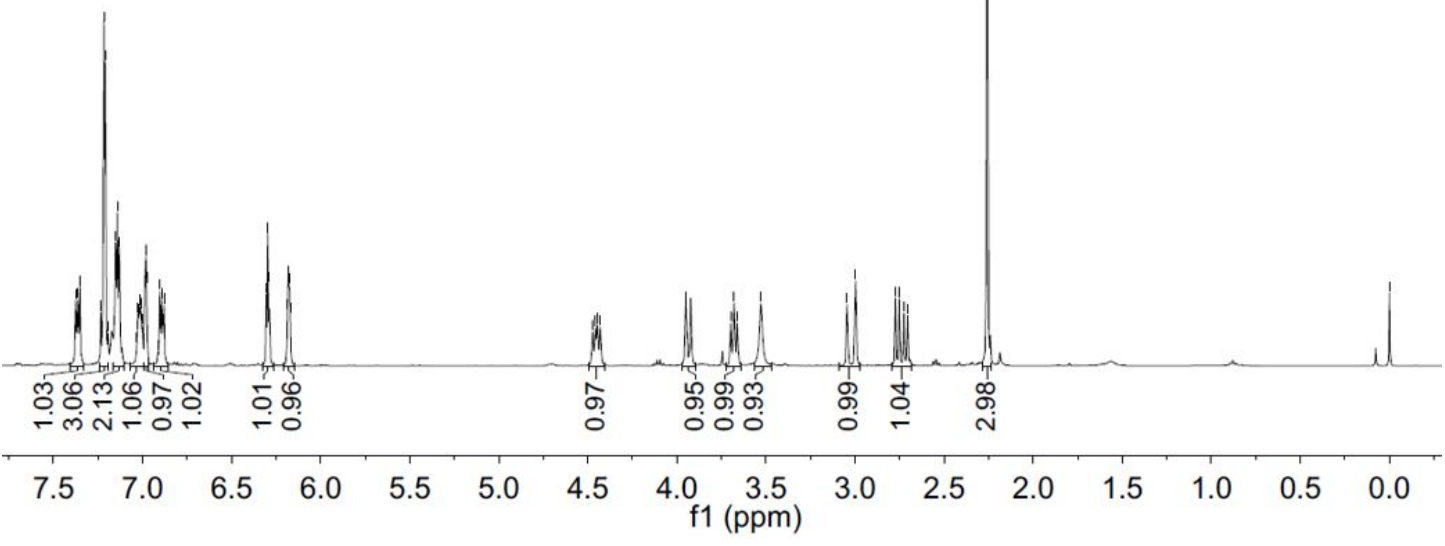

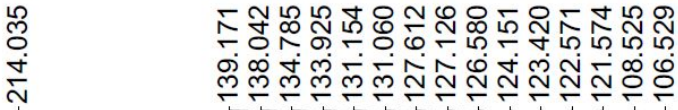

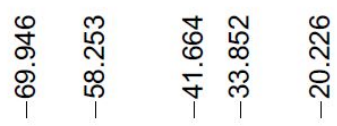

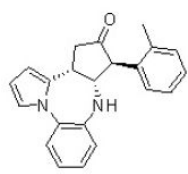

3ba

$210200190180170160150140130120 \begin{gathered}11010090 \\ \mathrm{f} 1(\mathrm{ppm})\end{gathered}$ 


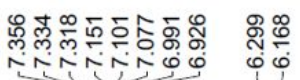

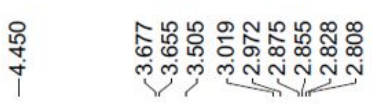
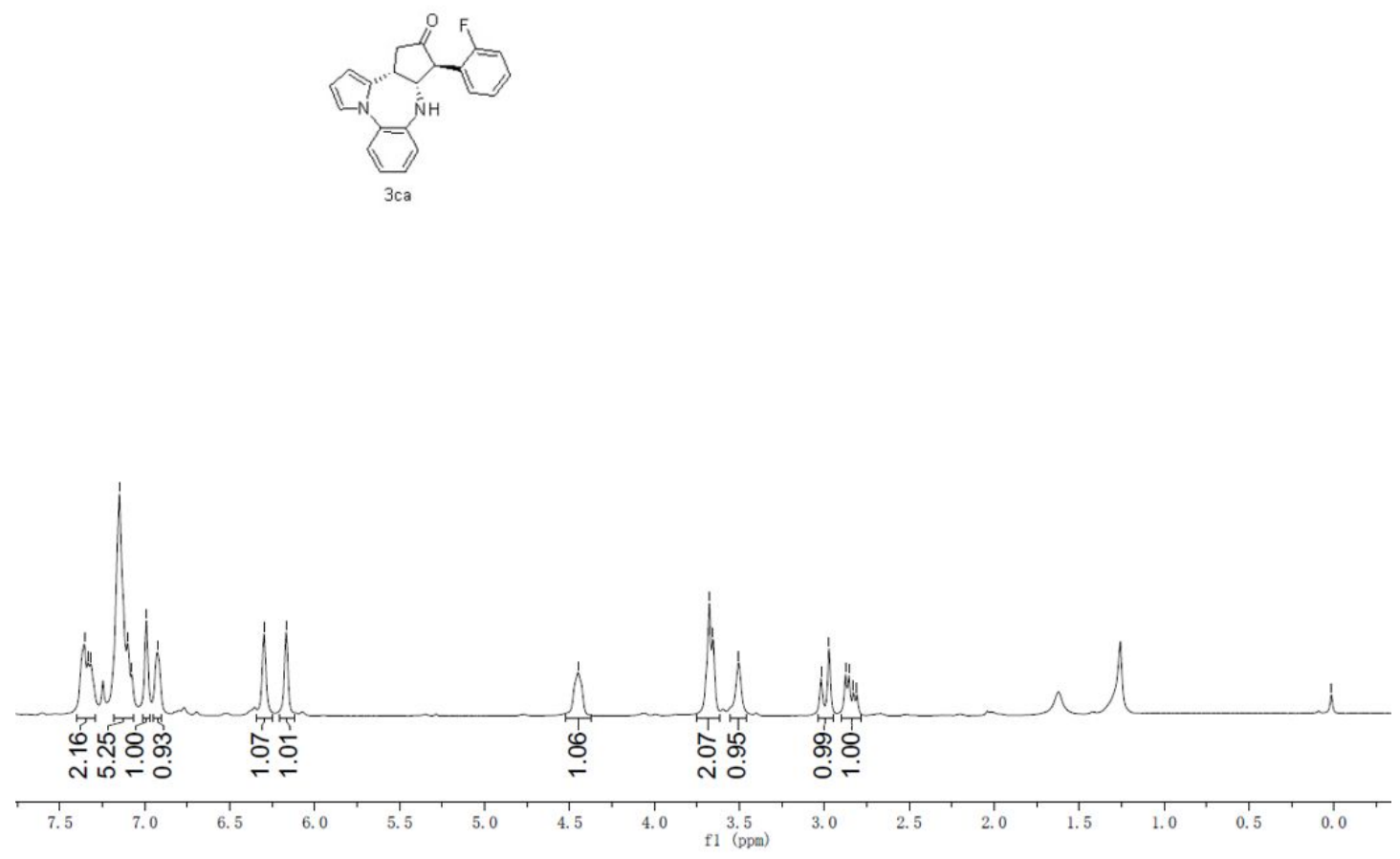

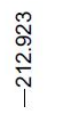

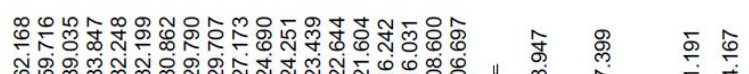

$$
\begin{aligned}
& \text { i, }
\end{aligned}
$$

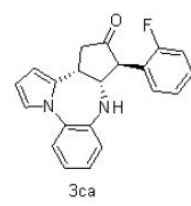

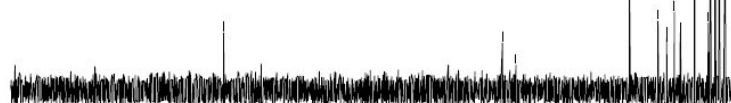

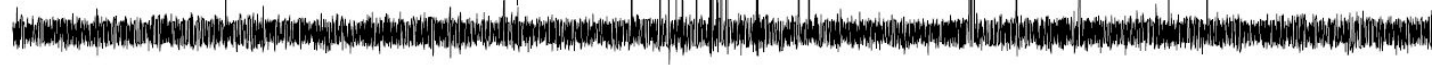

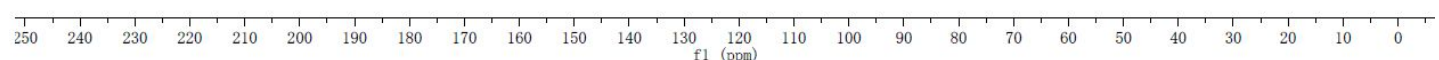




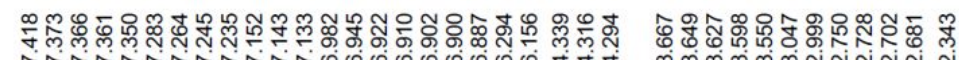

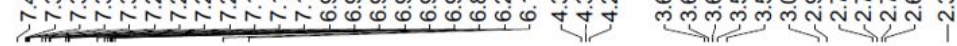

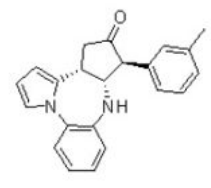

3da
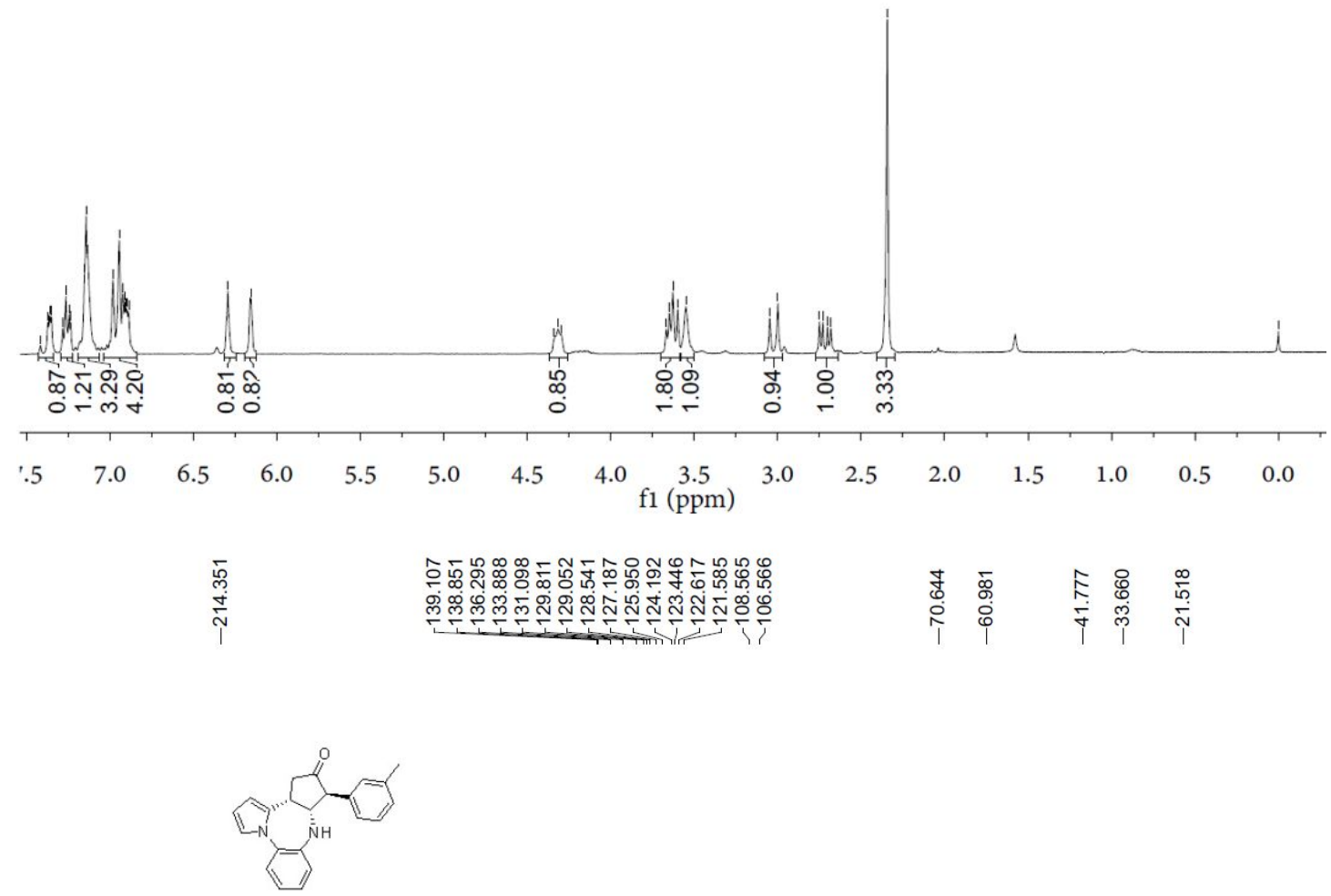

3da

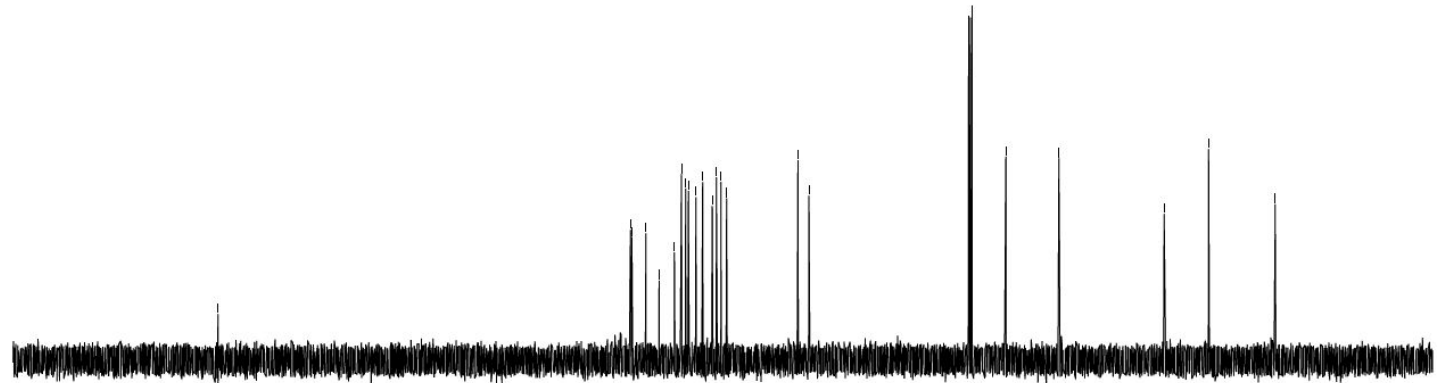

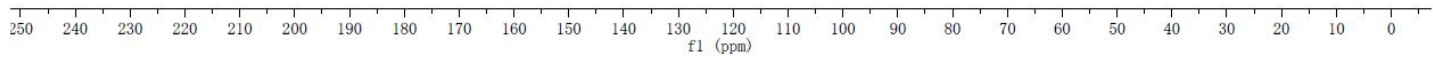




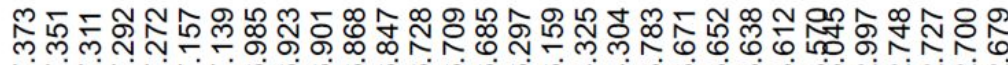

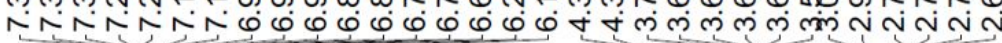

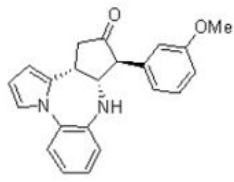

3ea
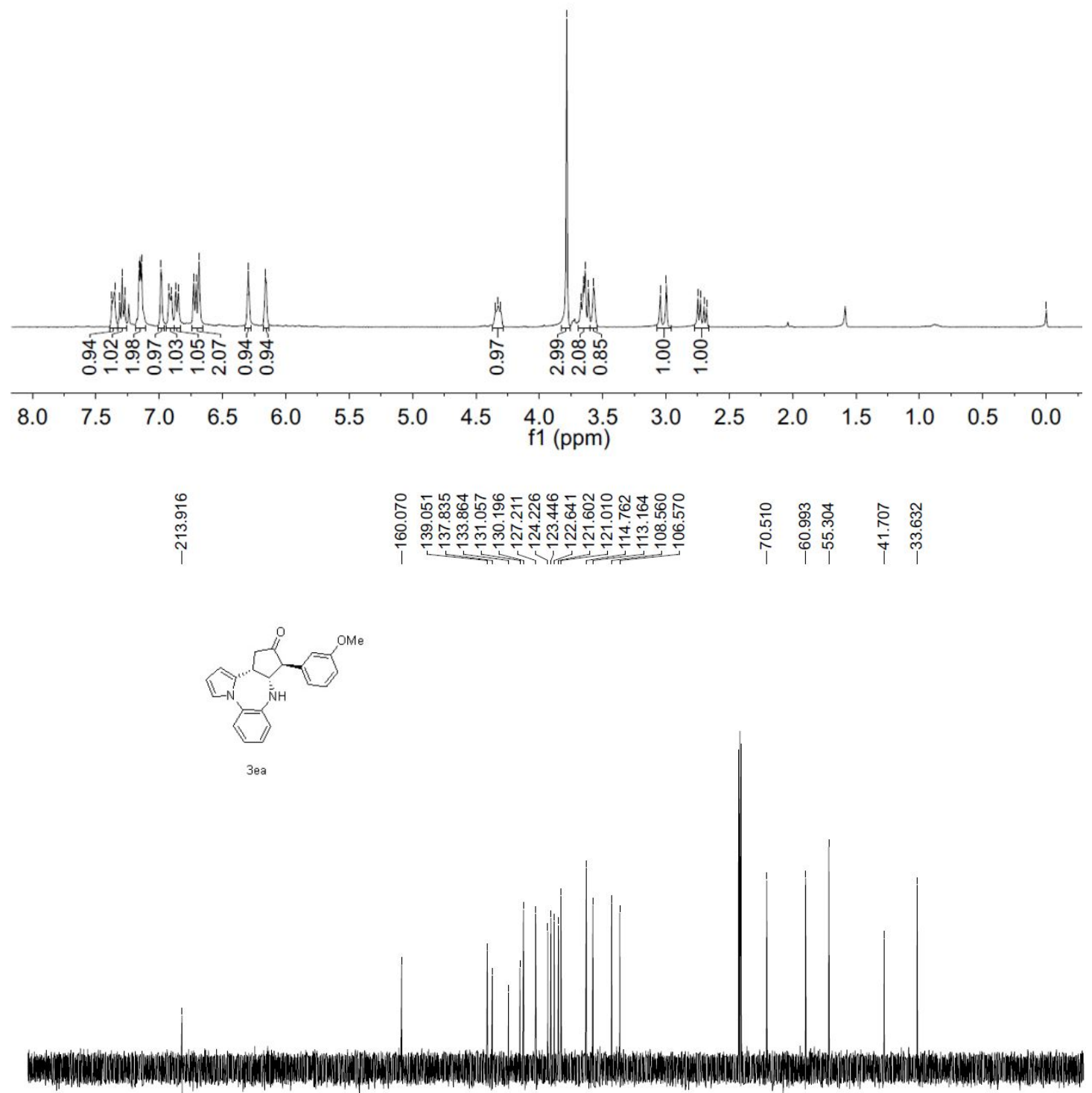

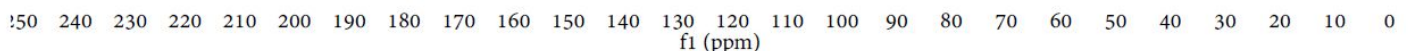



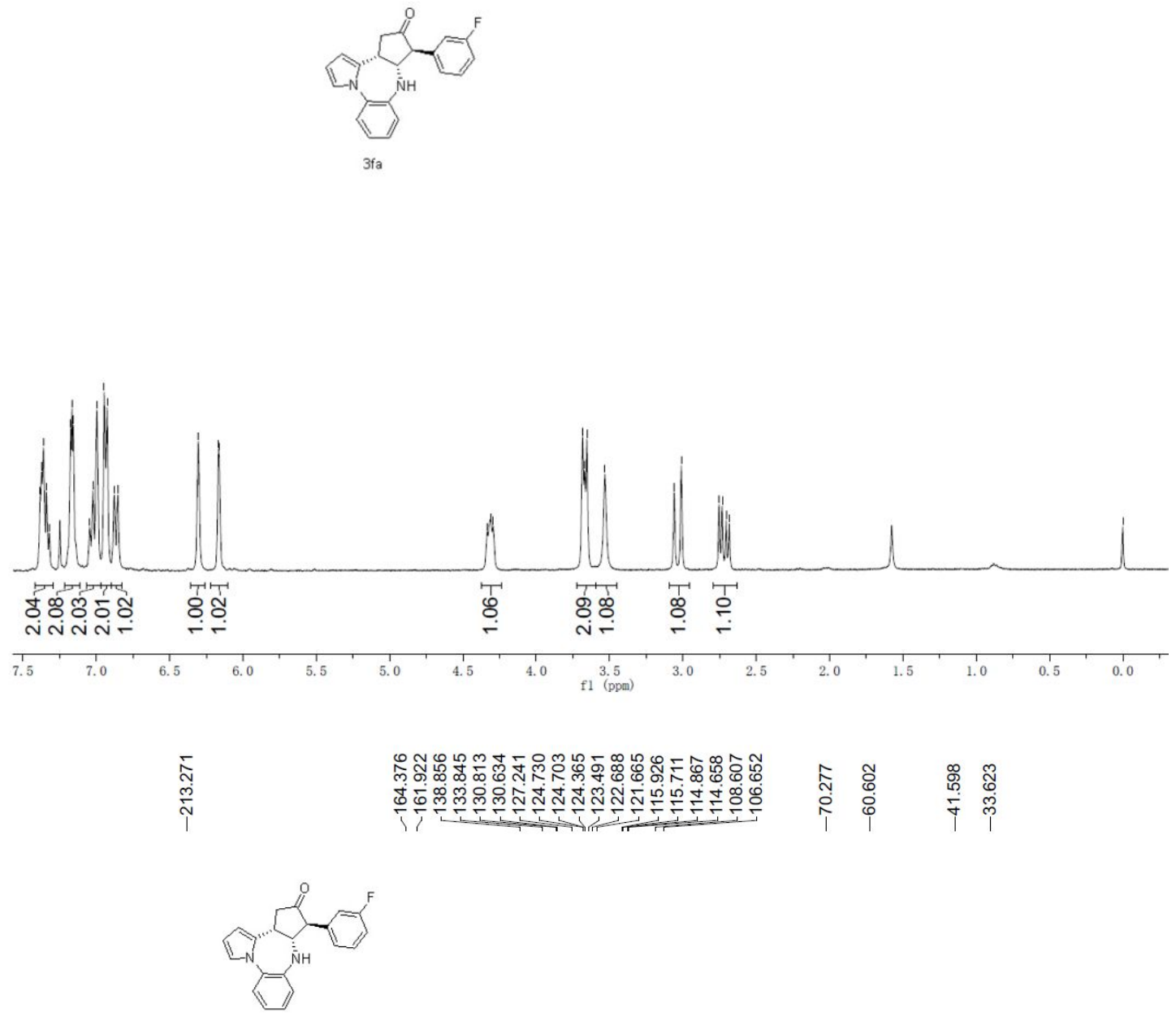

$3 \mathrm{fa}$

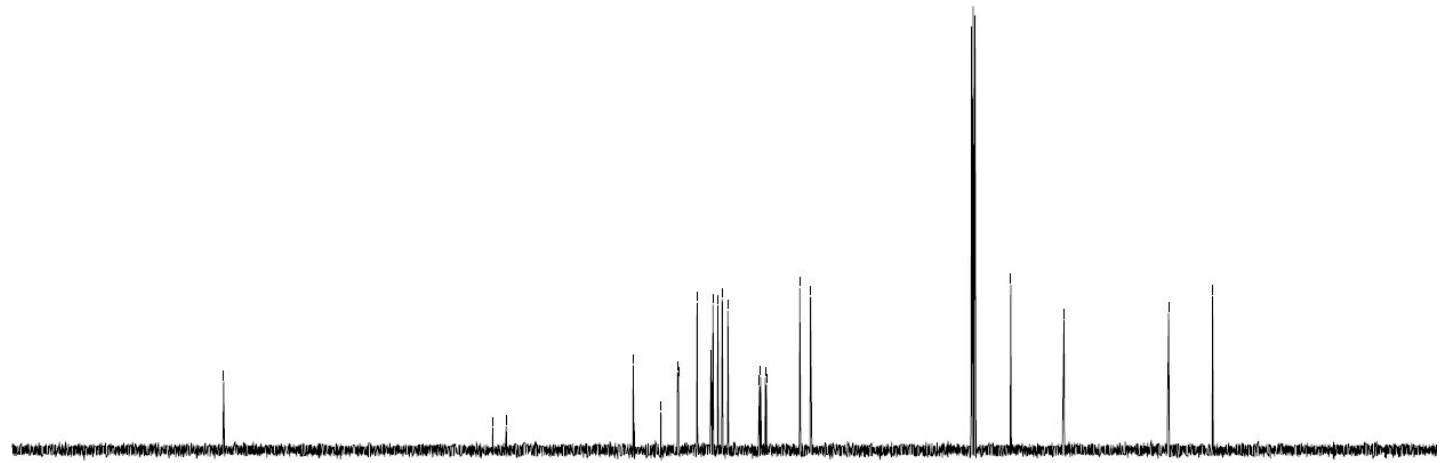

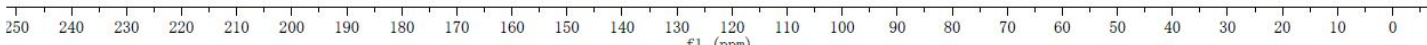



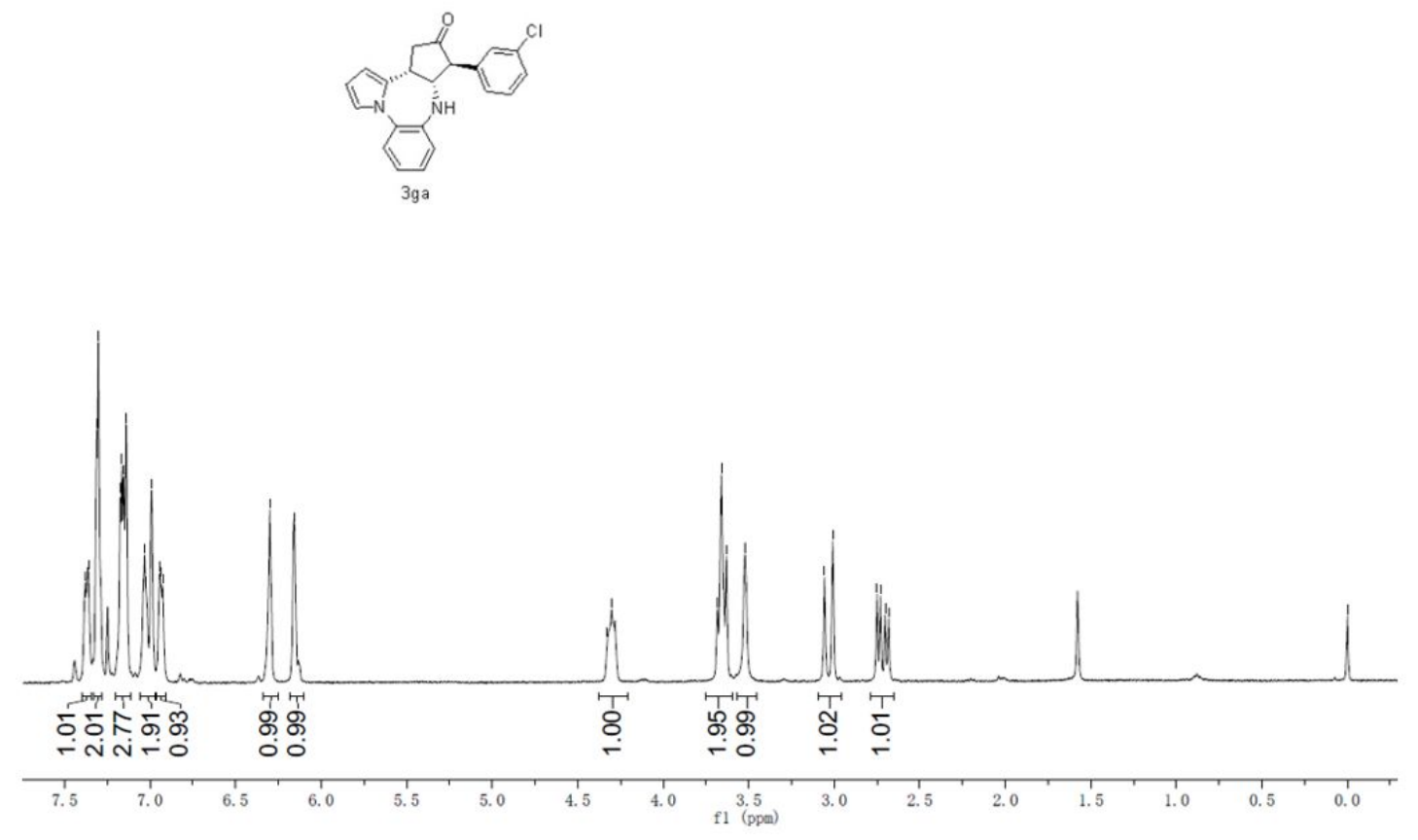

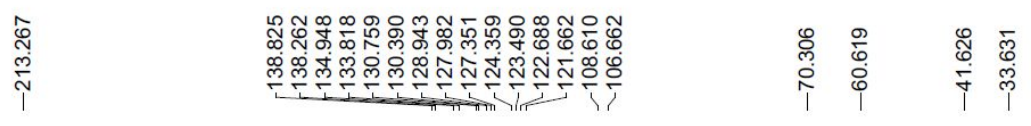

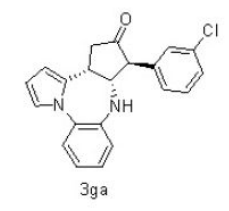

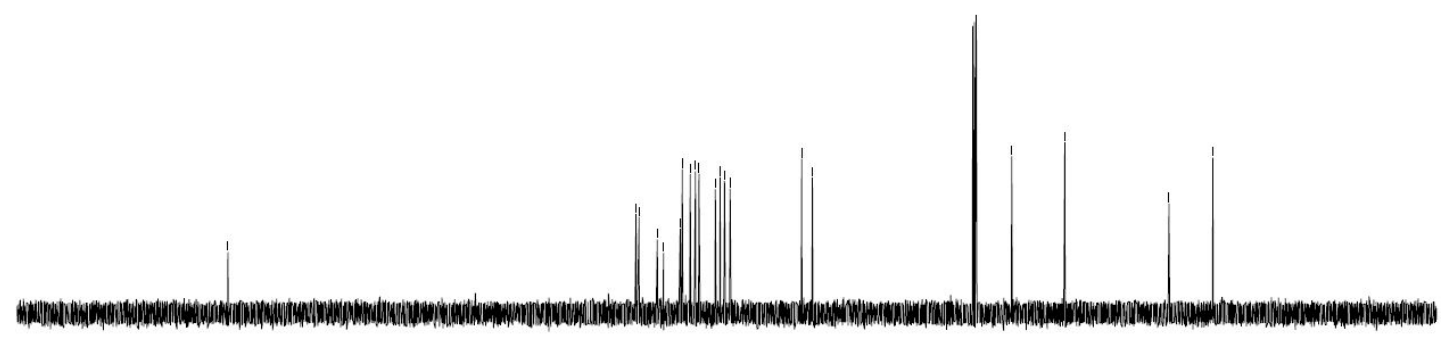

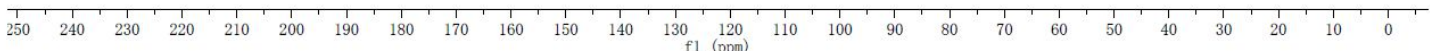



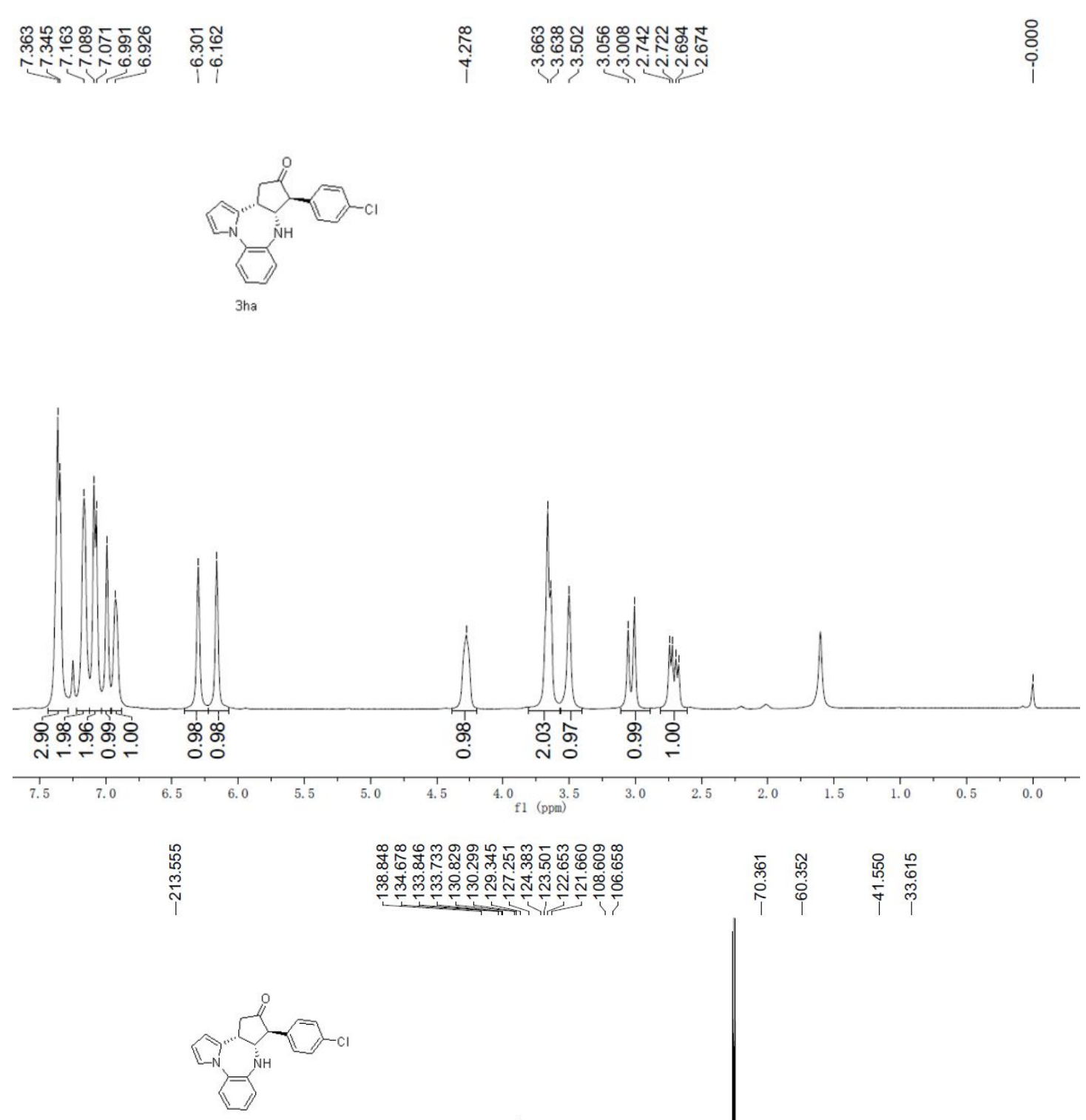

3ha

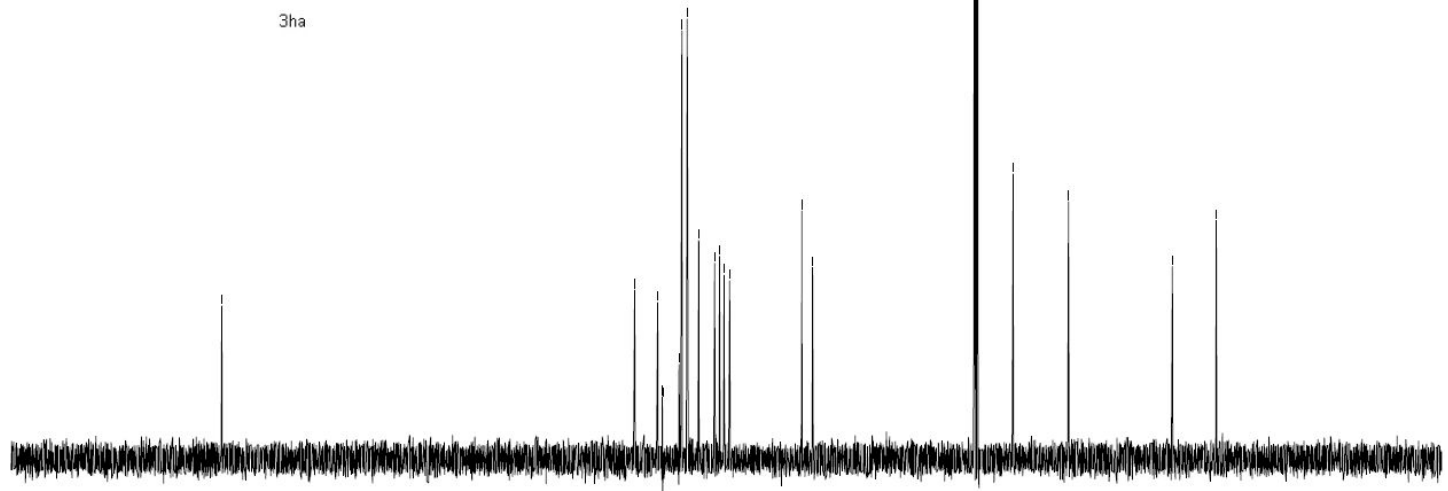

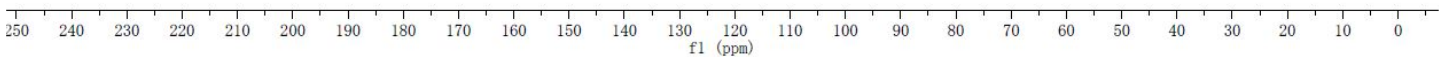




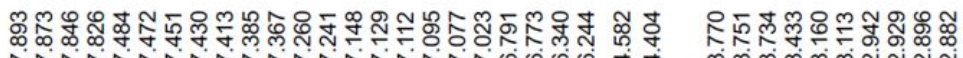

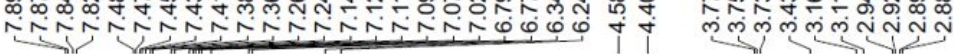

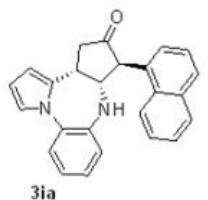

3ia

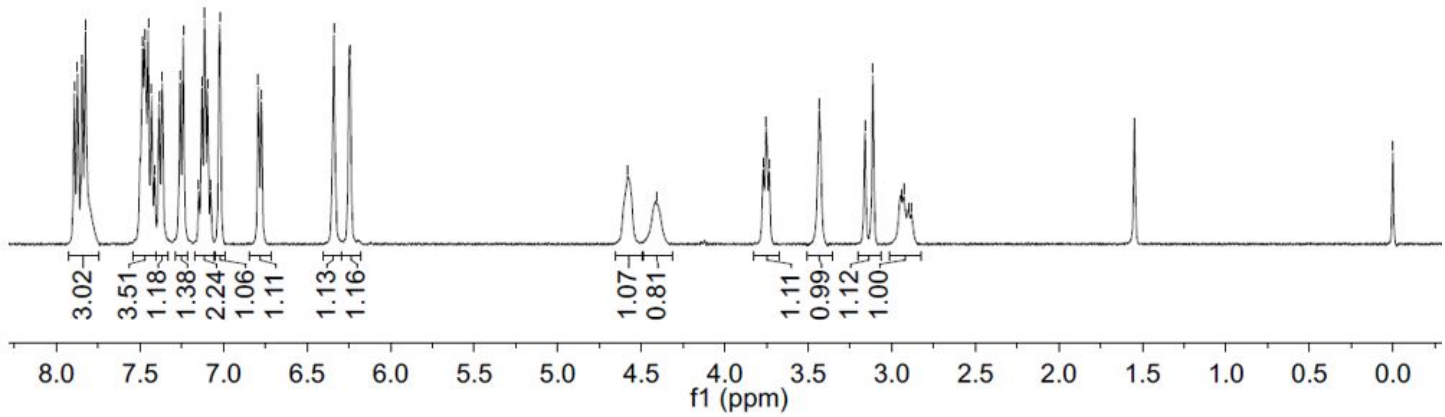

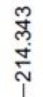

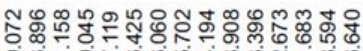

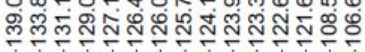

站

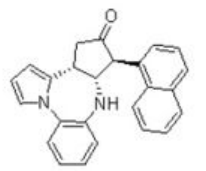

3ia ${ }^{13} \mathrm{C}$ NMR

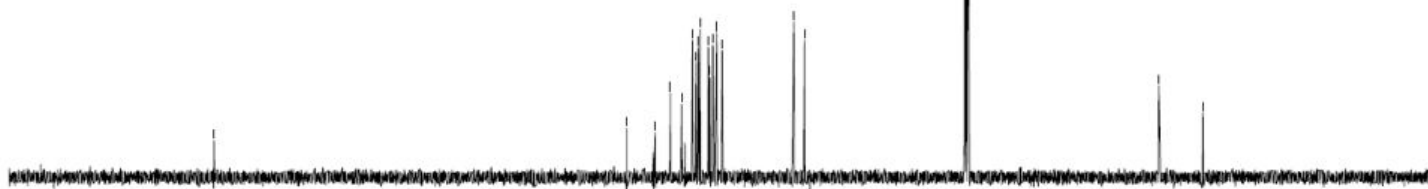

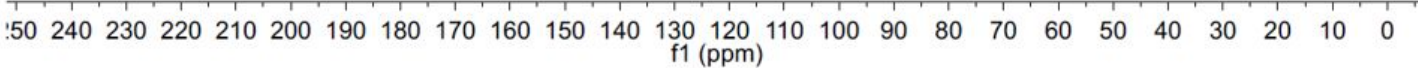




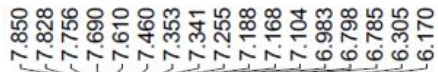

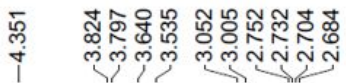

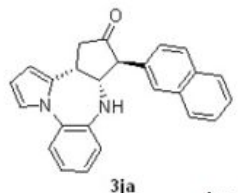

${ }^{1} \mathrm{H}$ NMR

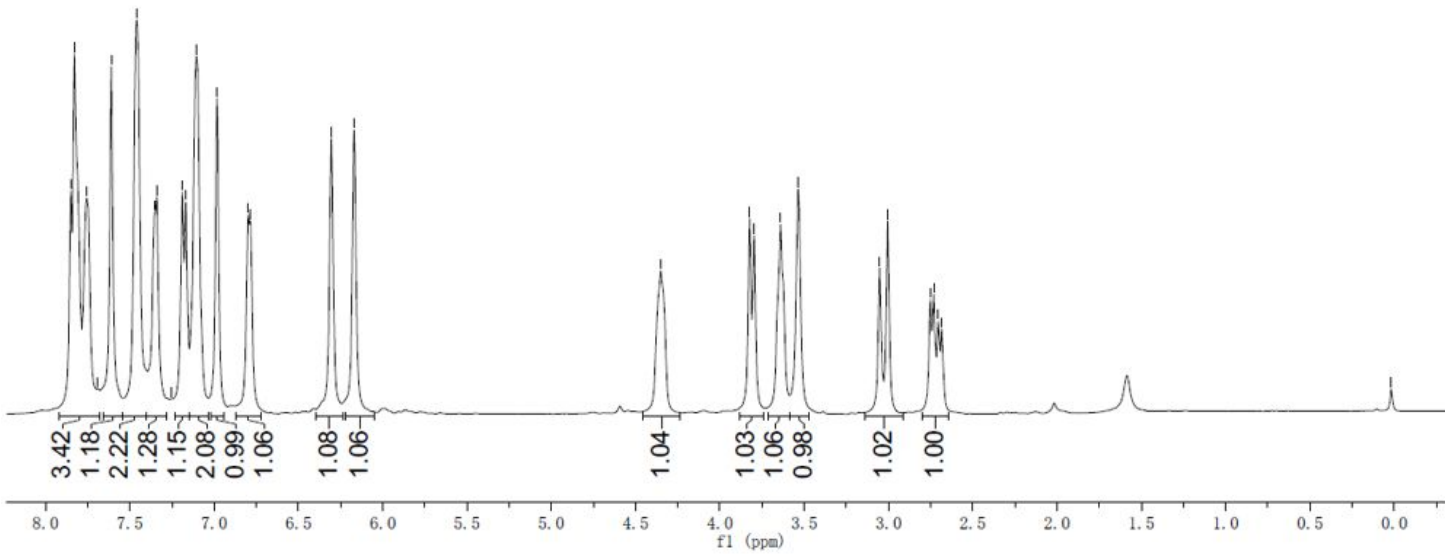

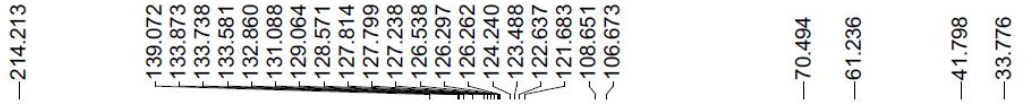
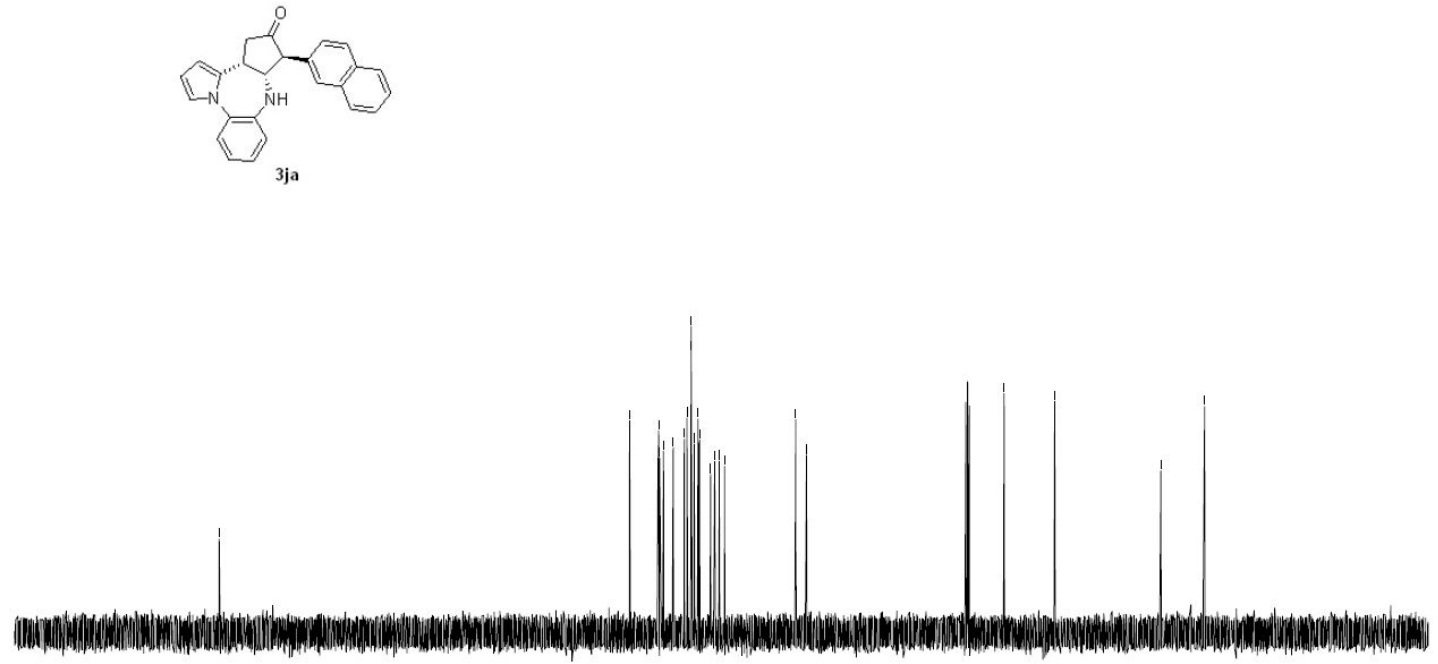

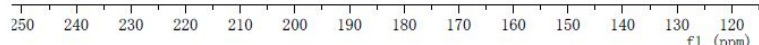




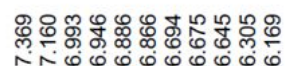

ن

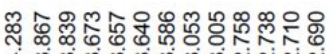

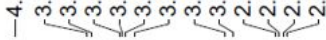

웅
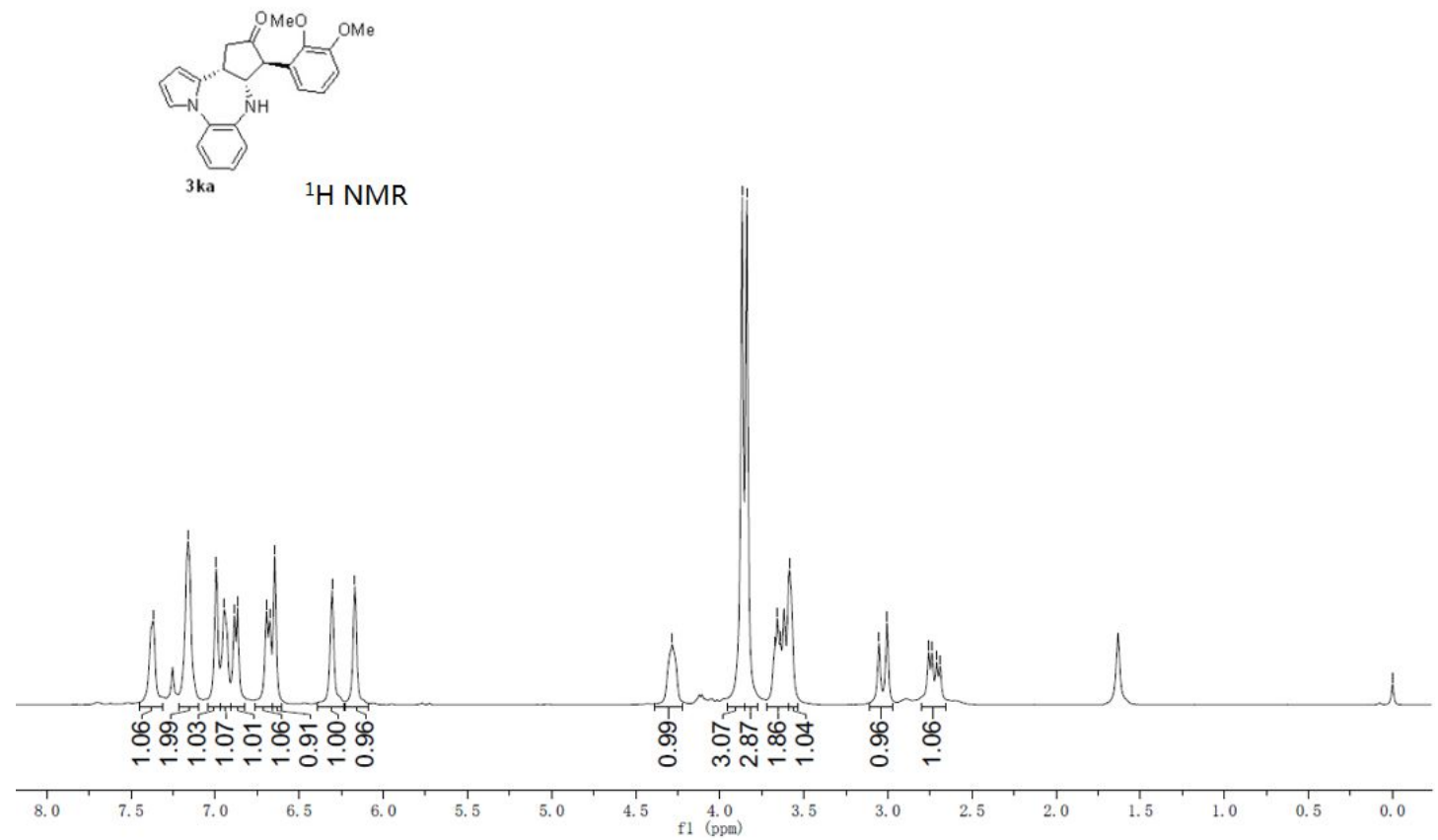

ำ

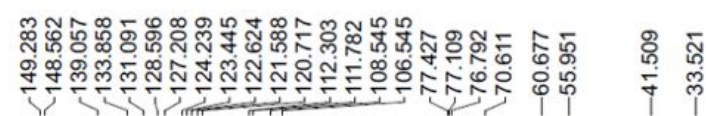

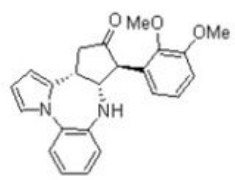

$3 \mathrm{ka}{ }^{13} \mathrm{C}$ NMR

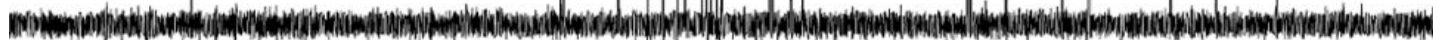

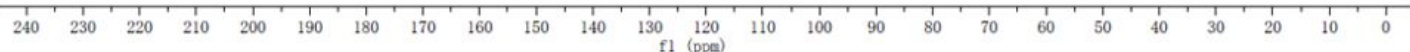

f1 $\left(\begin{array}{l}120 \\ \text { ppa })\end{array}\right.$ 


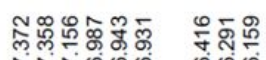

Nivio

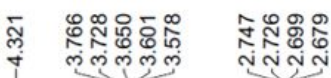

$\stackrel{8}{0}$

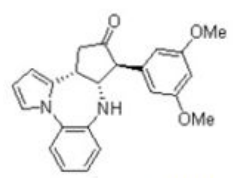

3la ${ }^{1} \mathrm{H} \mathrm{NMR}$
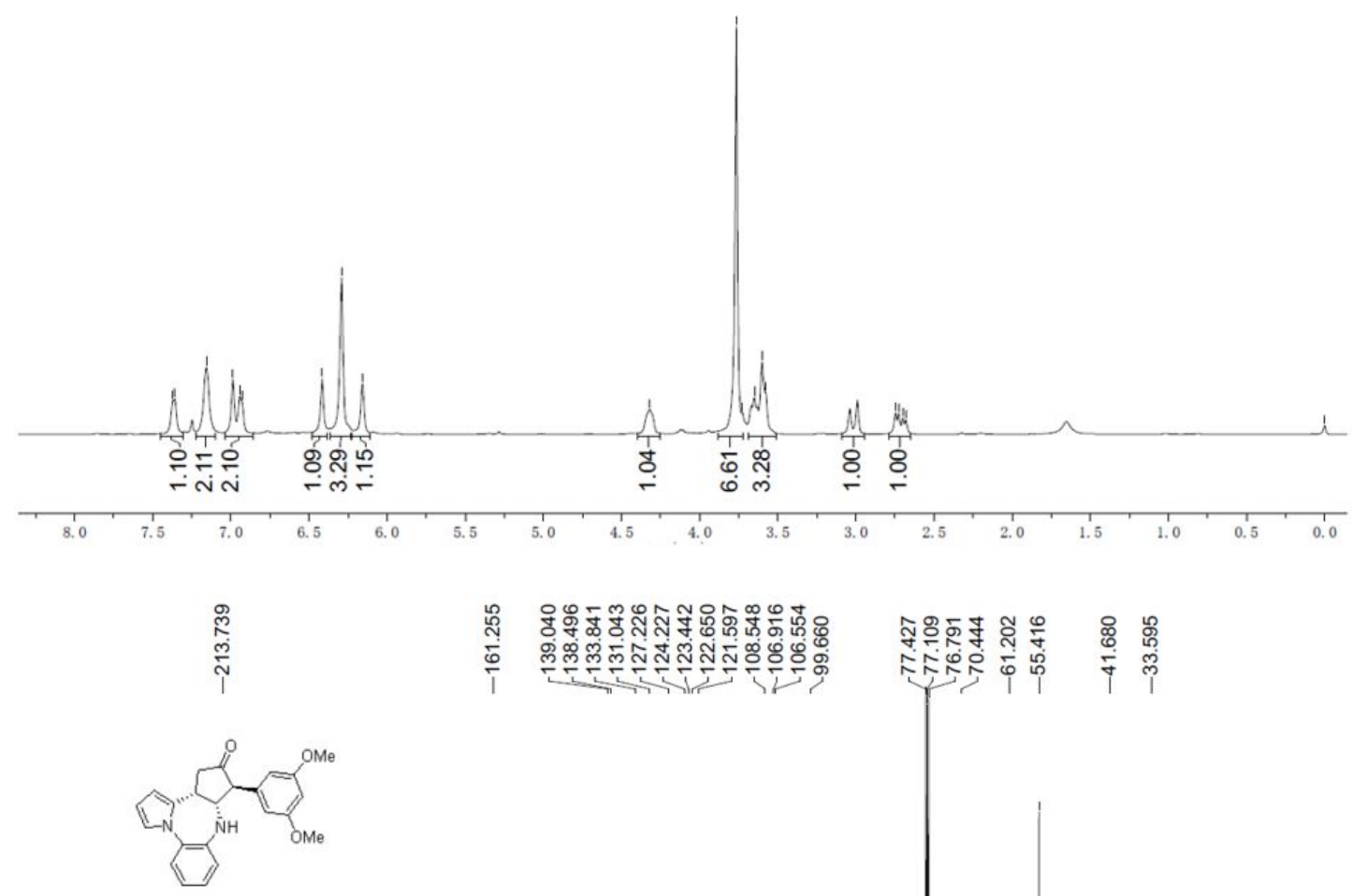

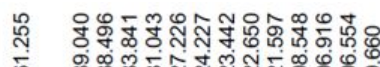

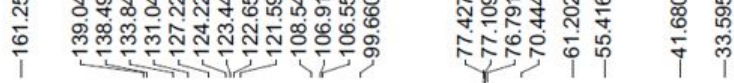

3la ${ }^{13} \mathrm{C}$ NMR

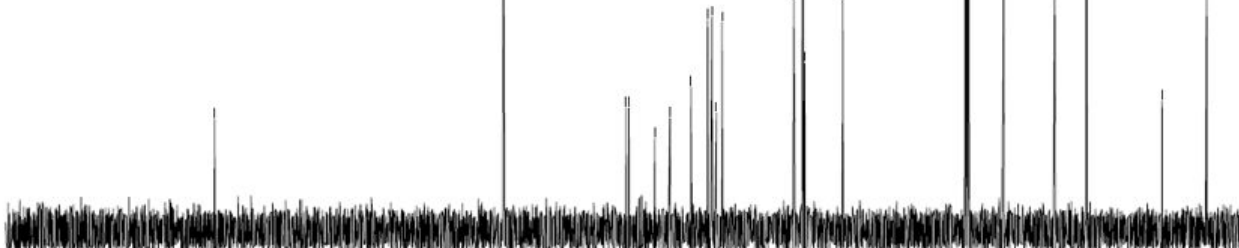

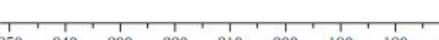
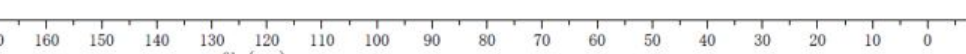


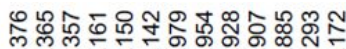

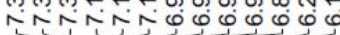

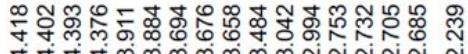
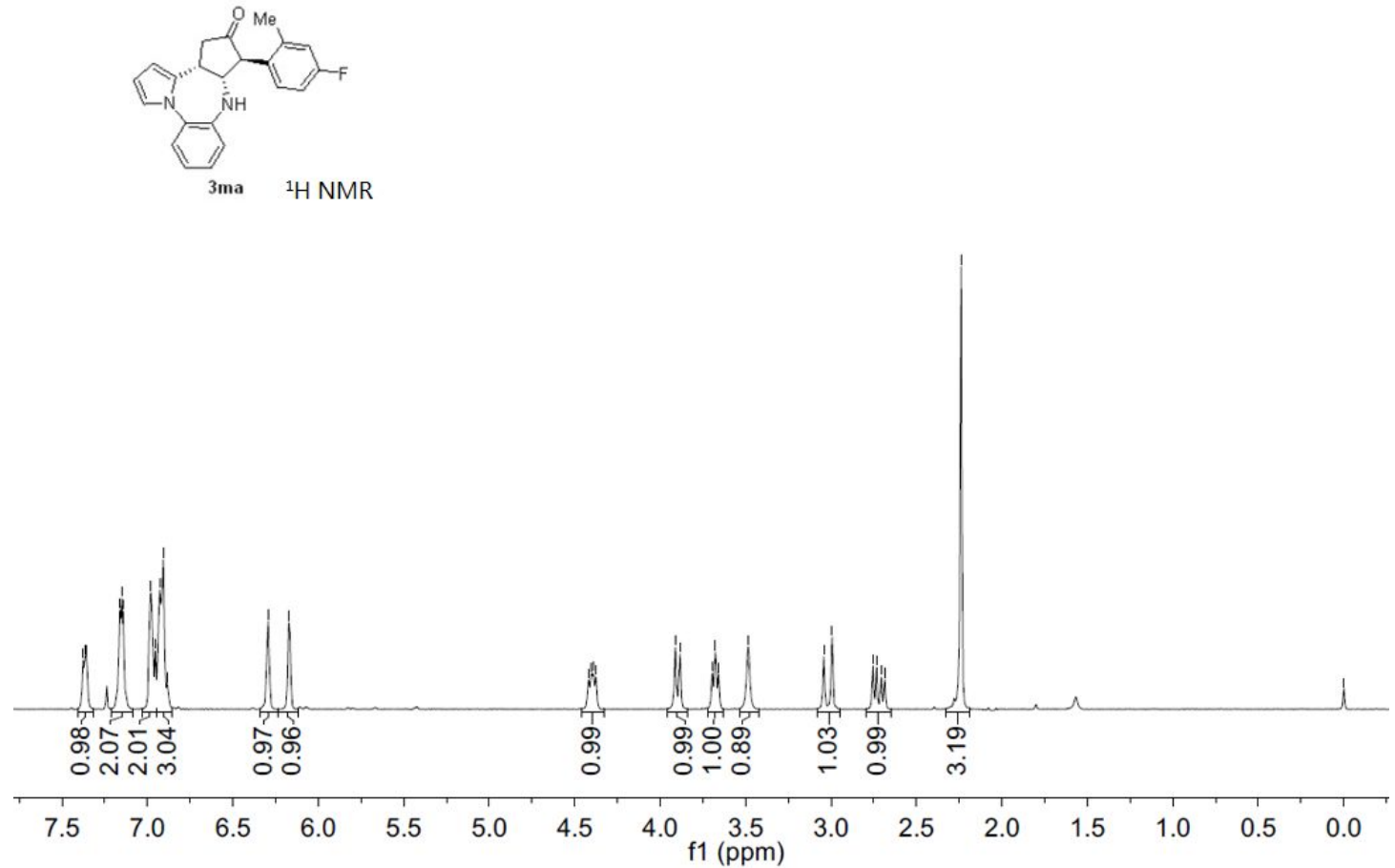

$\bar{i}$
$\stackrel{\sim}{N}$

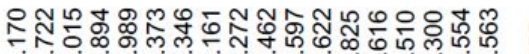

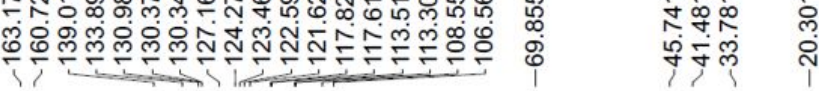
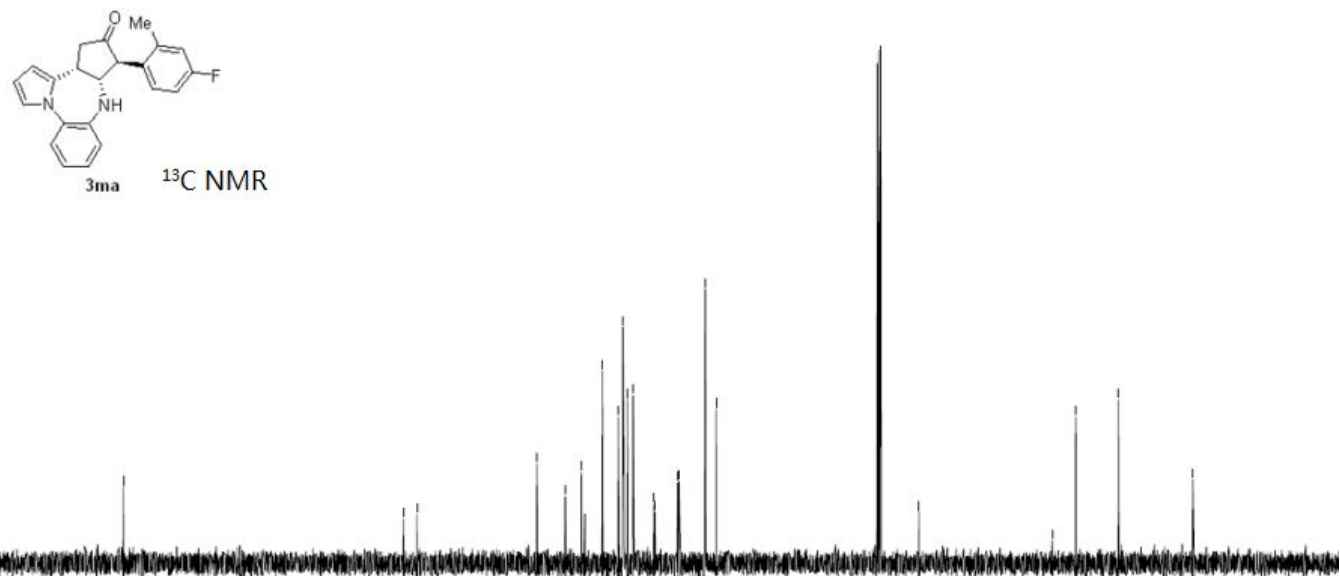

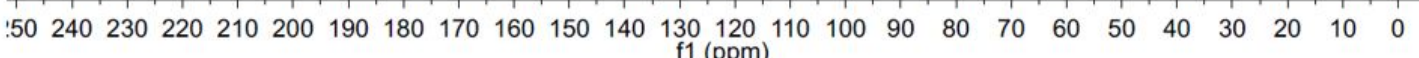



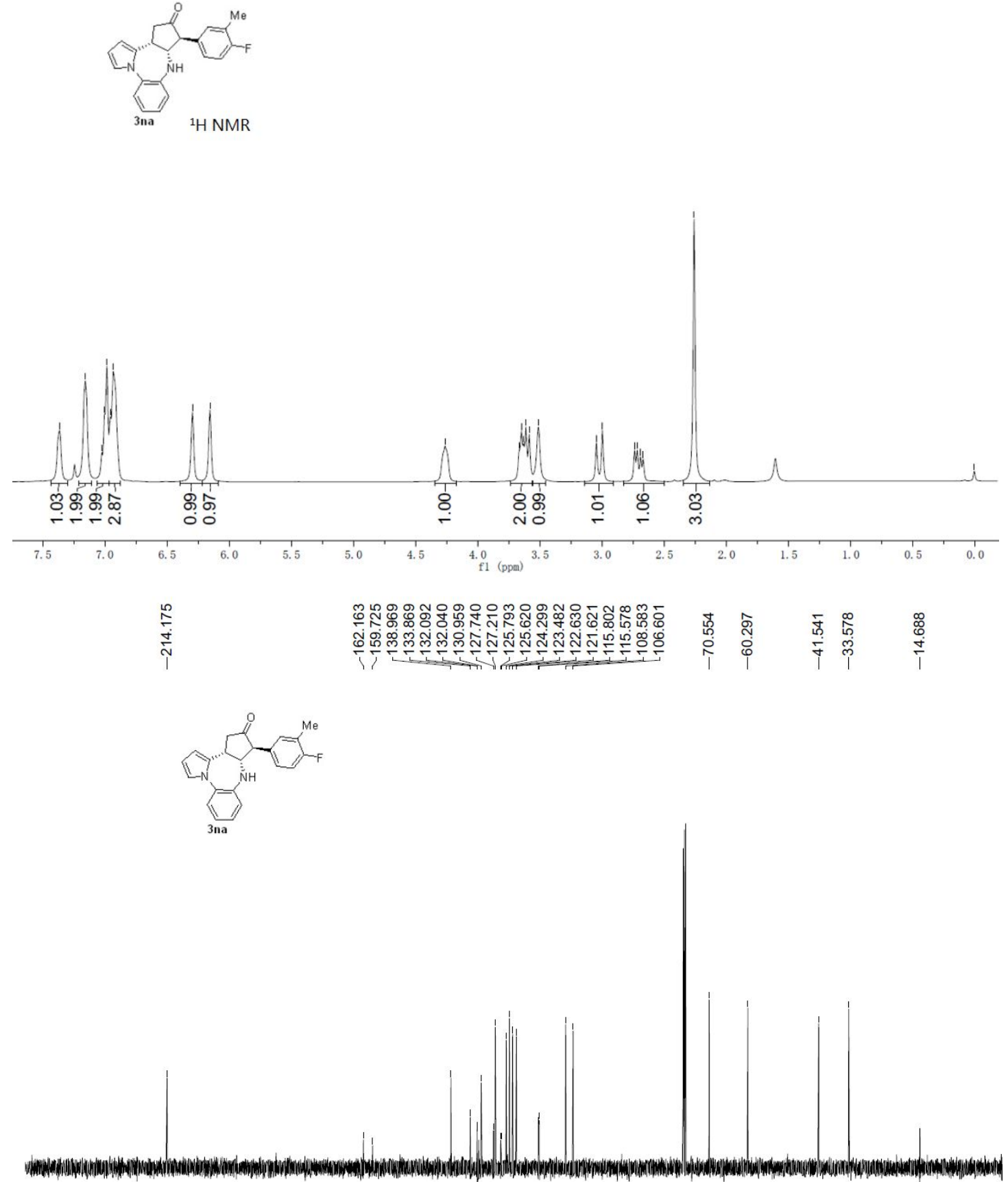

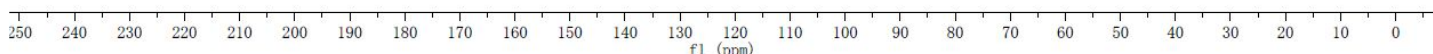




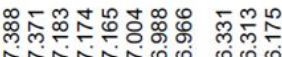

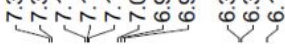

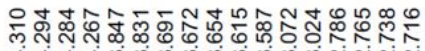

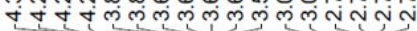

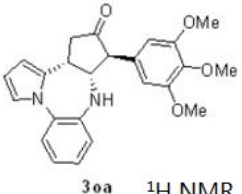

3oa ${ }^{1} \mathrm{H}$ NMR
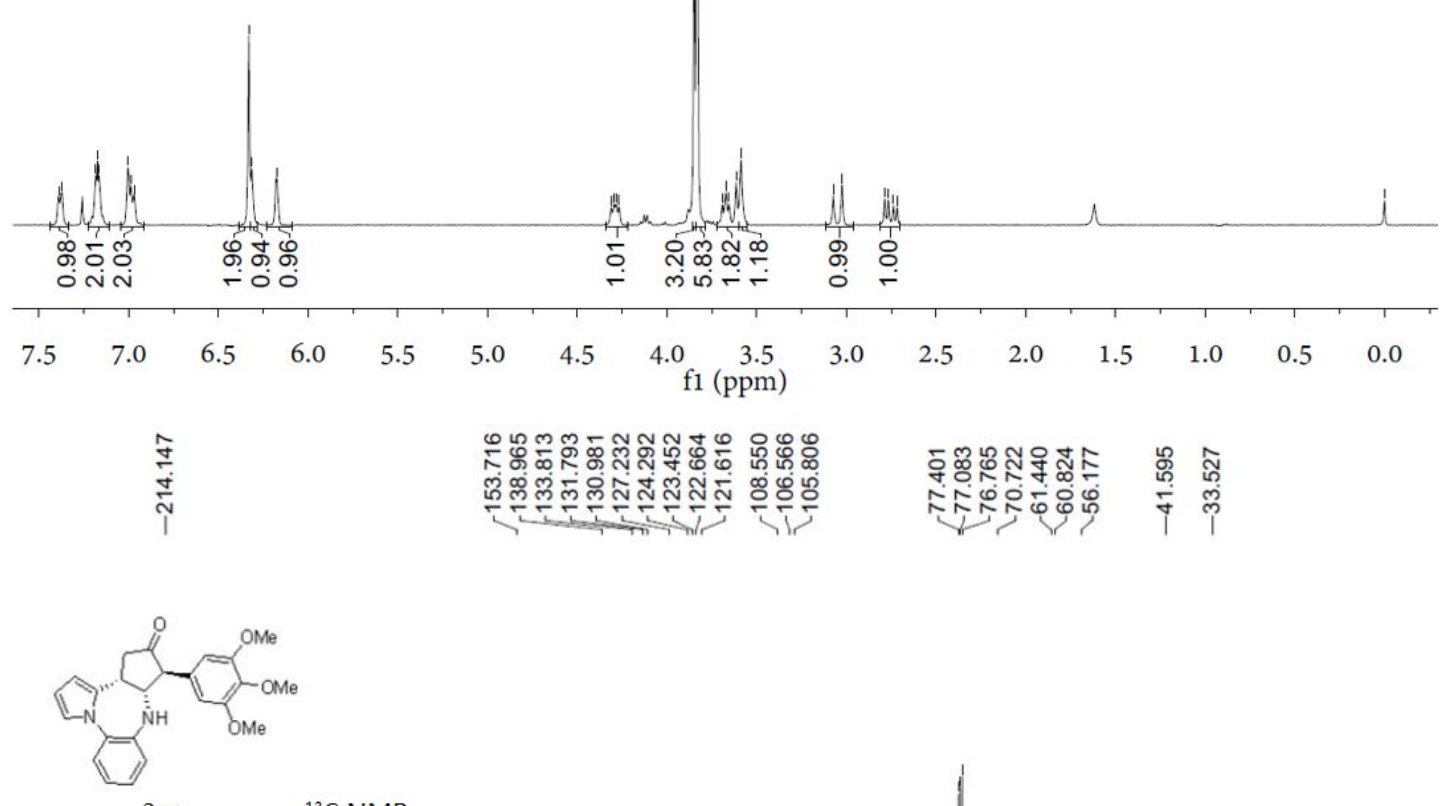

3oa $\quad{ }^{13} \mathrm{C}$ NMR

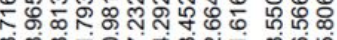

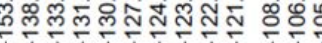

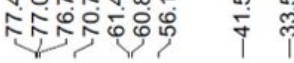

C NMR

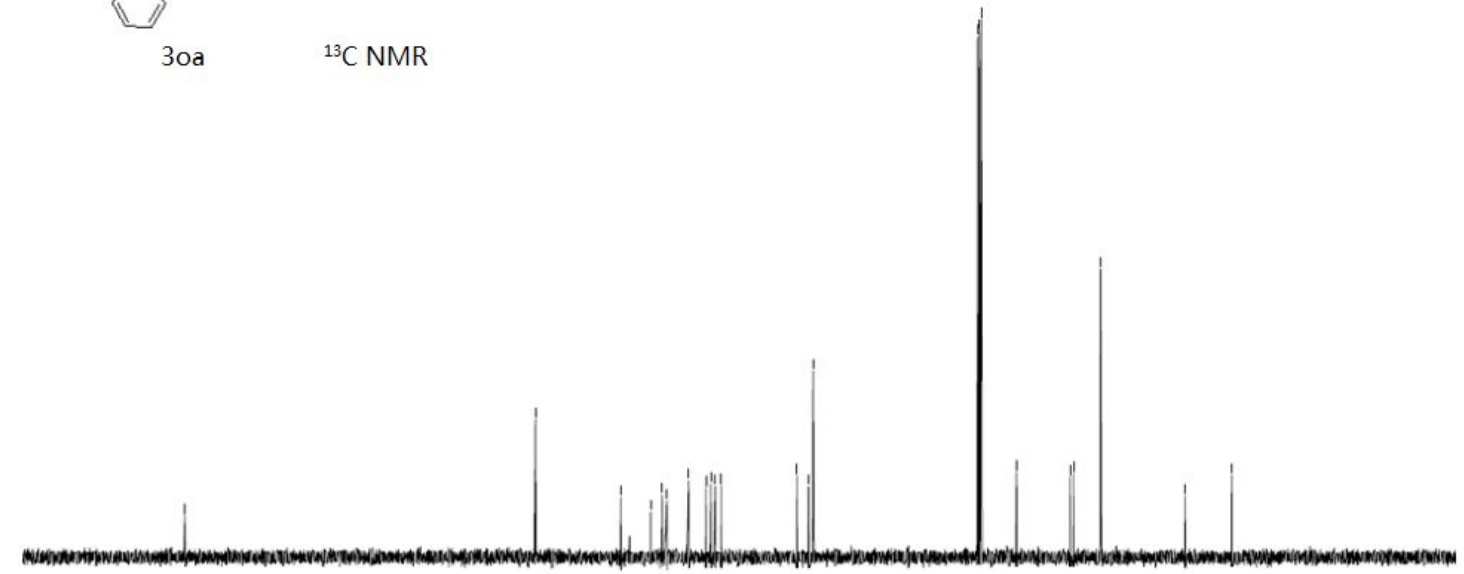

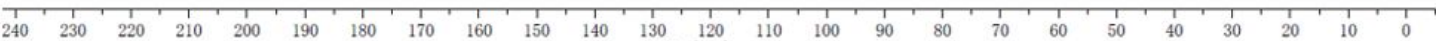



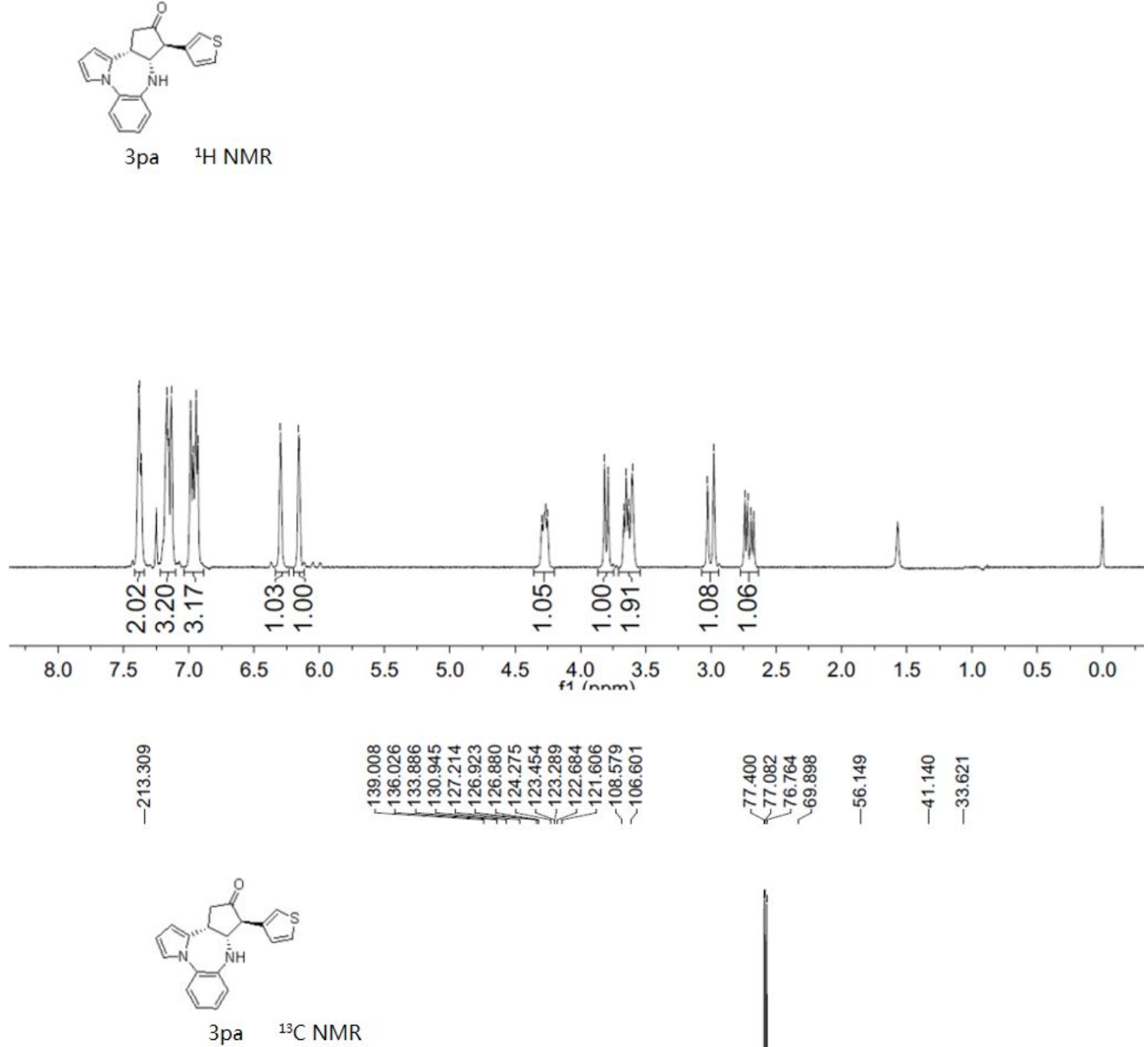

3pa $\quad{ }^{13} \mathrm{C}$ NMR

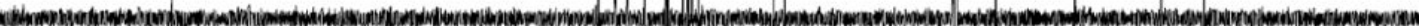

$24023022021020019018017016015014013012011010090 \quad 80 \quad 70 \quad 60 \quad 50 \quad 40 \quad 30 \quad 20 \quad 10 \quad 0$ f1 (ppm) 


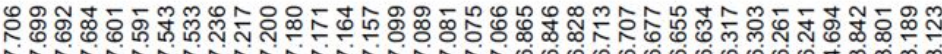

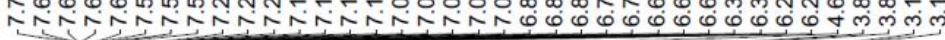

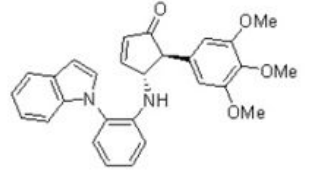

3ob $\quad{ }^{1} \mathrm{H} N M R$

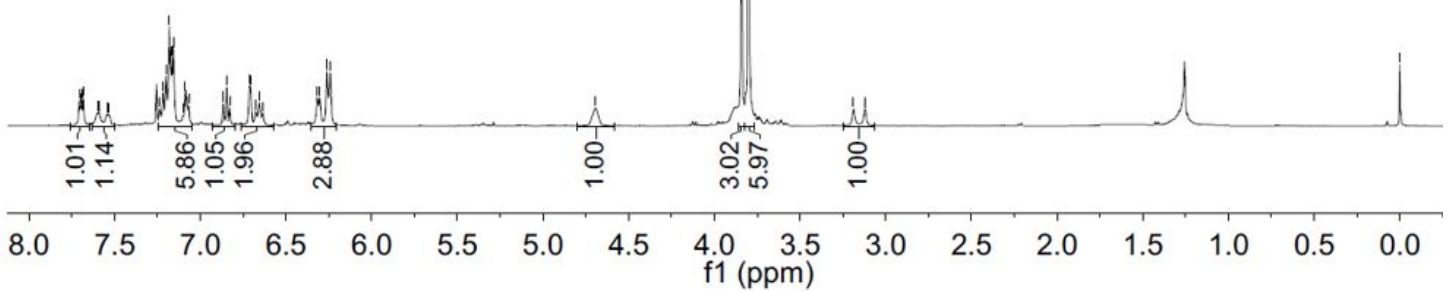


న
ஸे
ஸे

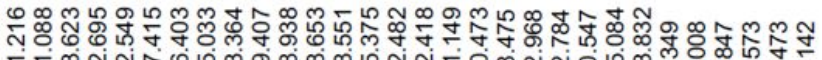

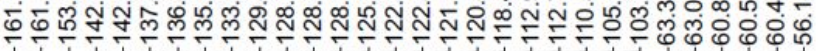

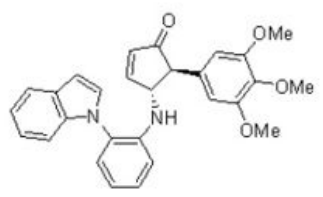

3ob $\quad{ }^{13} \mathrm{C}$ NMR

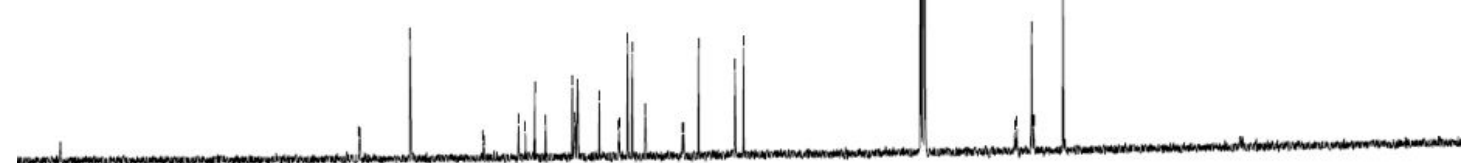

?10 $200190180170160150140130120 \begin{gathered}110 \\ \mathrm{f} 1(\mathrm{ppm})\end{gathered}$ 


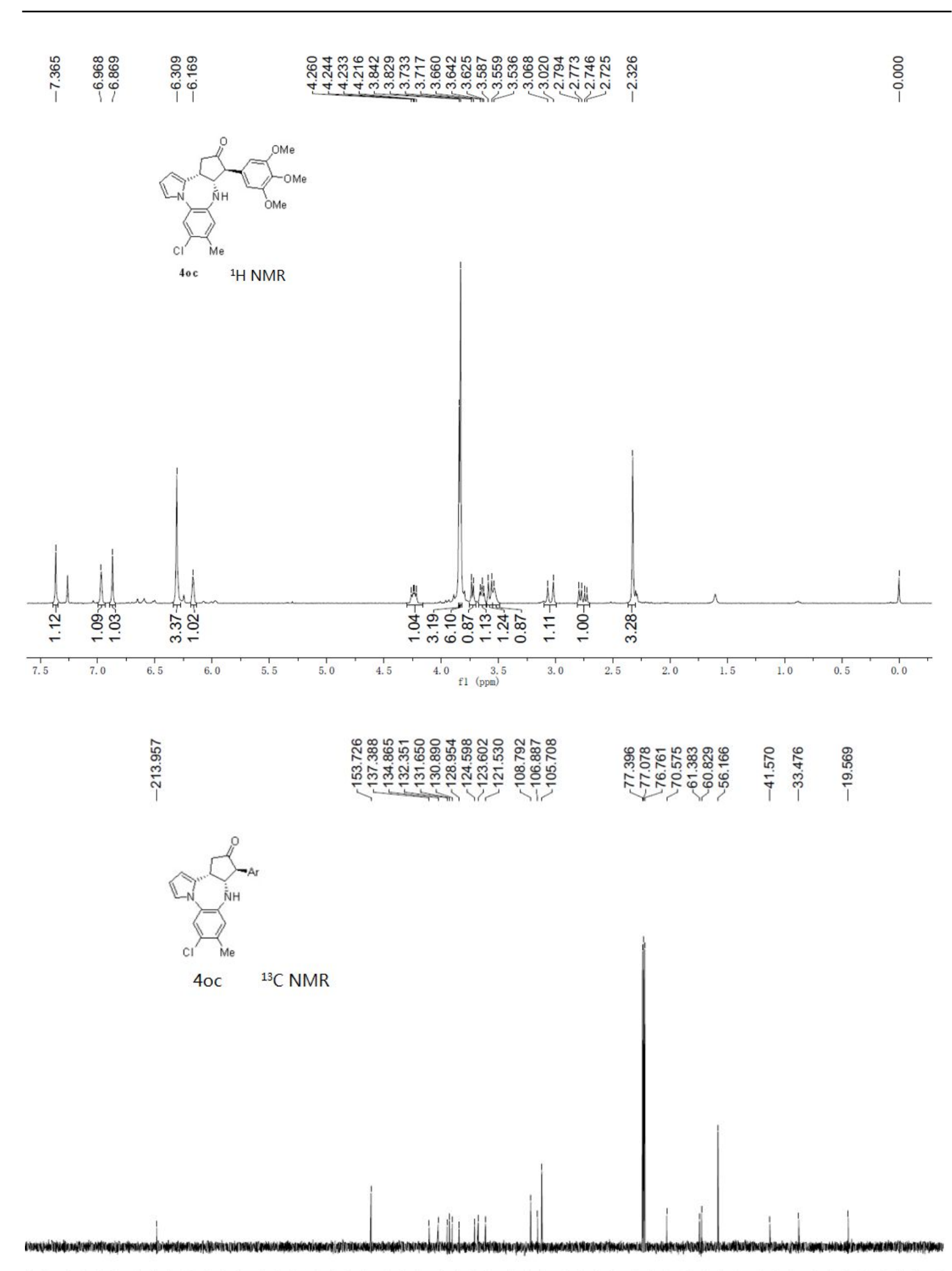

$5024023022021020019018017016015014013012011010090 \quad 80 \quad 70 \quad 60 \quad 50 \quad 40 \quad 30 \quad 20 \quad 10 \quad 0$ f1 (ppm) 


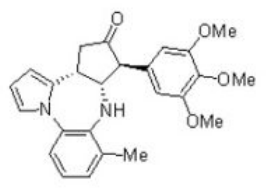

4oe ${ }^{1} \mathrm{H}$ NMR
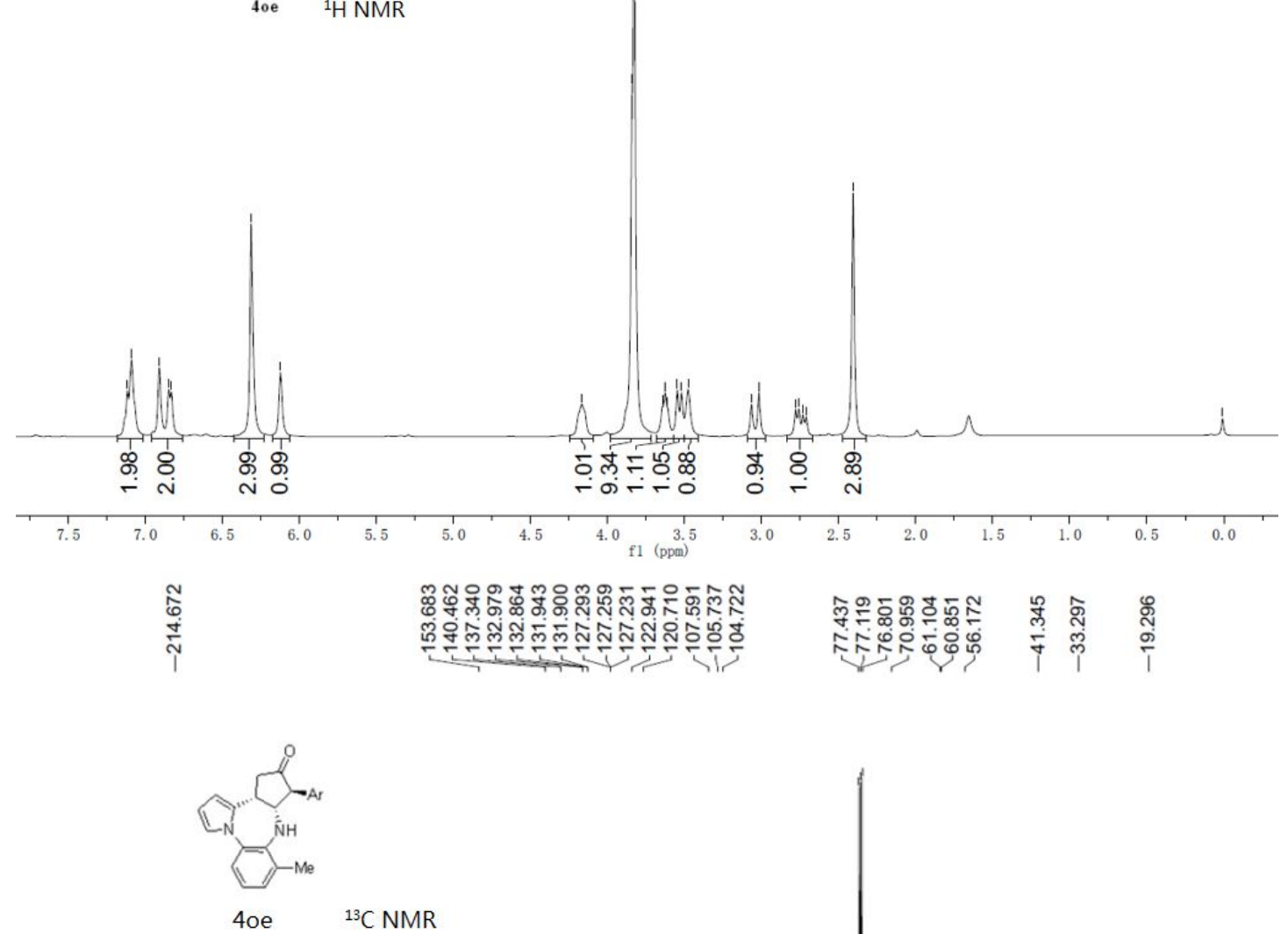

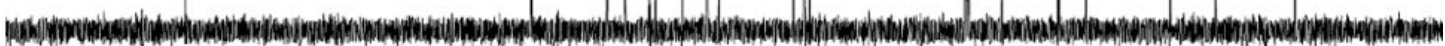

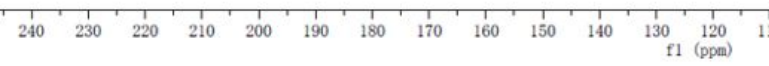




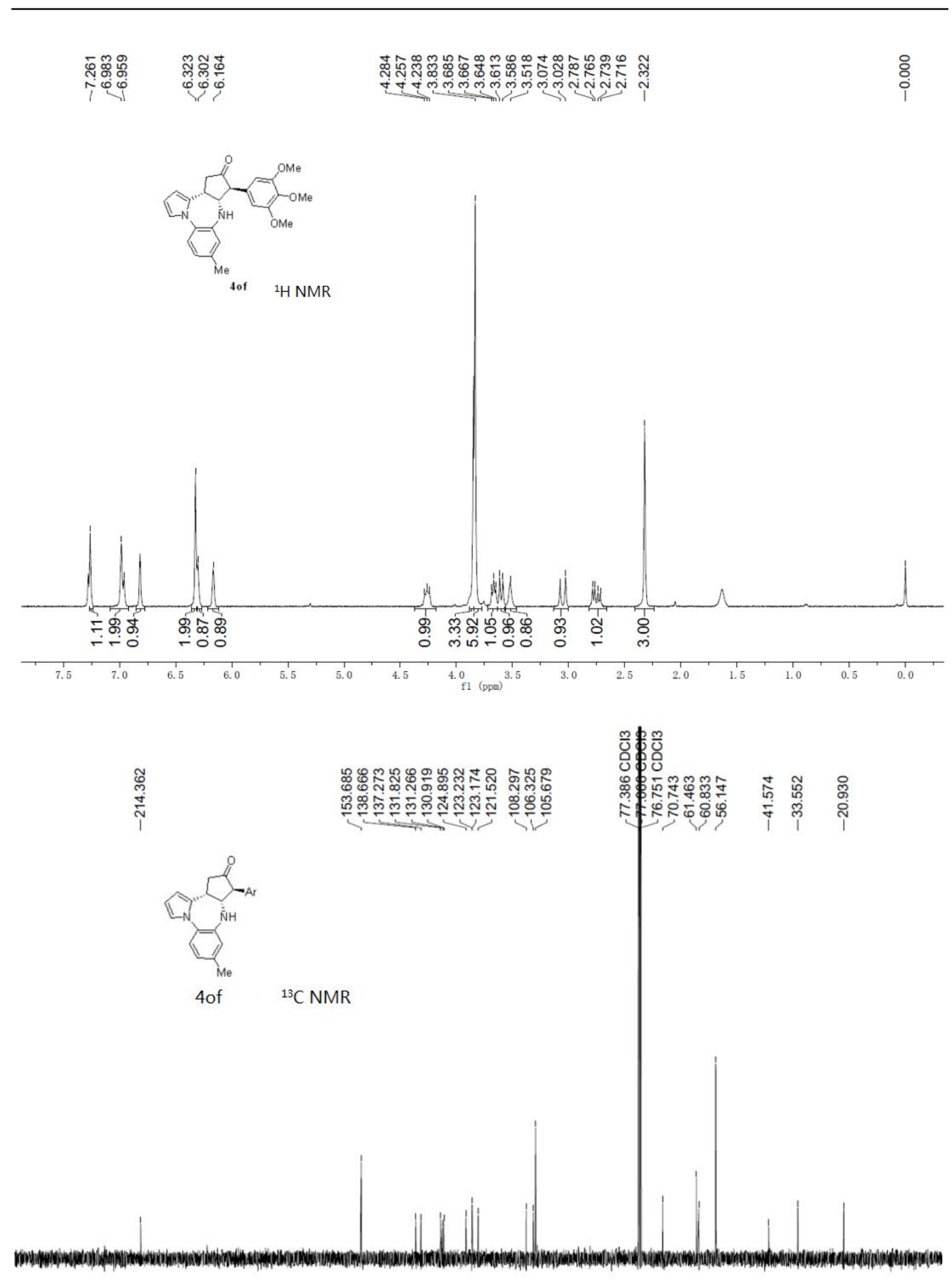

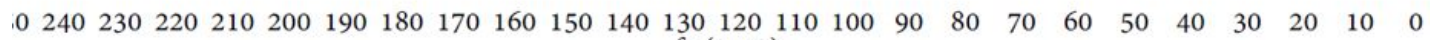
f1 (ppm) 


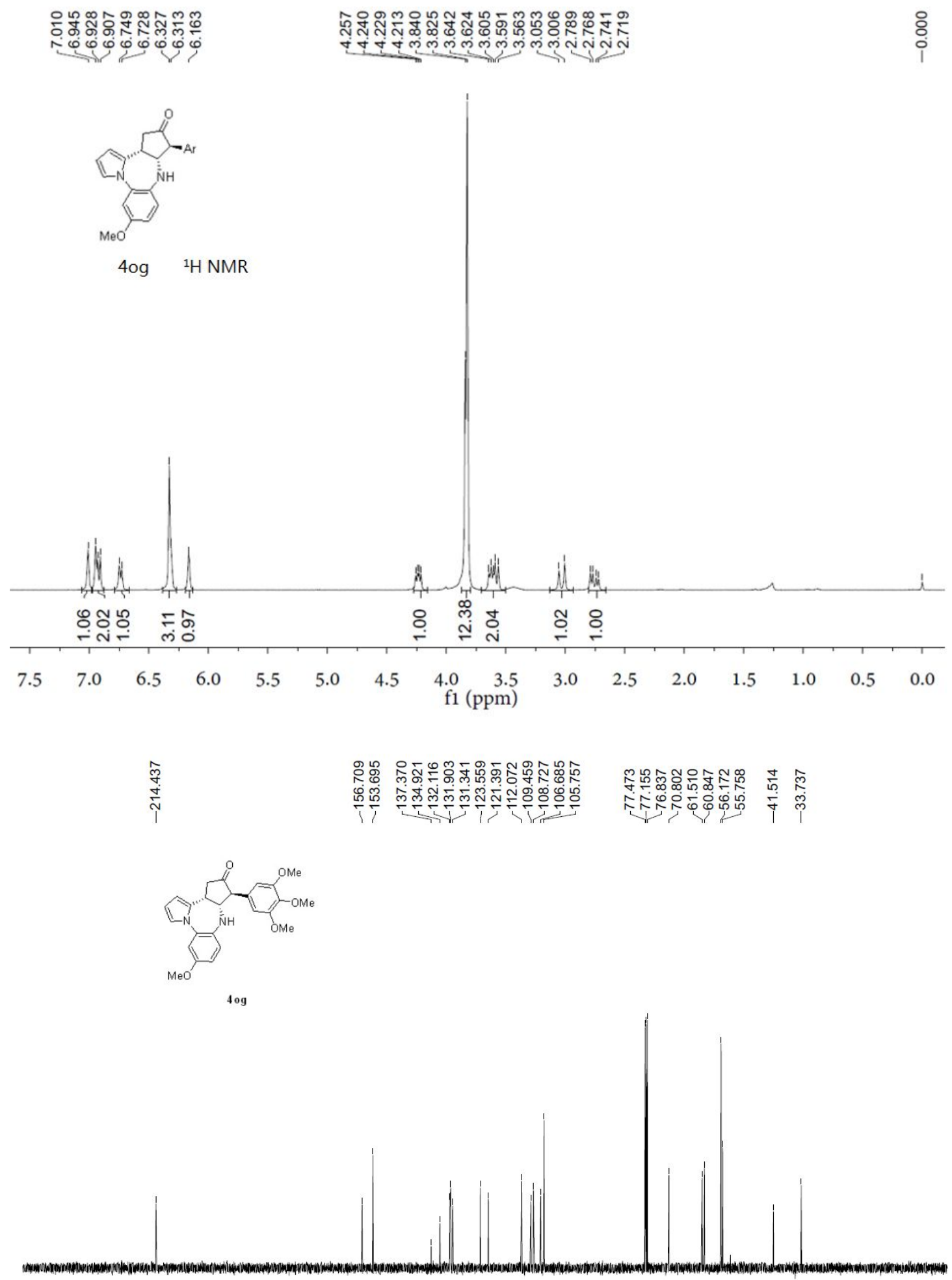

$\begin{array}{llllllllllllllllllllllllll}50 & 240 & 230 & 220 & 210 & 200 & 190 & 180 & 170 & 160 & 150 & 140 & 130 & 120 & 110 & 100 & 90 & 80 & 70 & 60 & 50 & 40 & 30 & 20 & 10 & 0\end{array}$ f1 (ppm) 


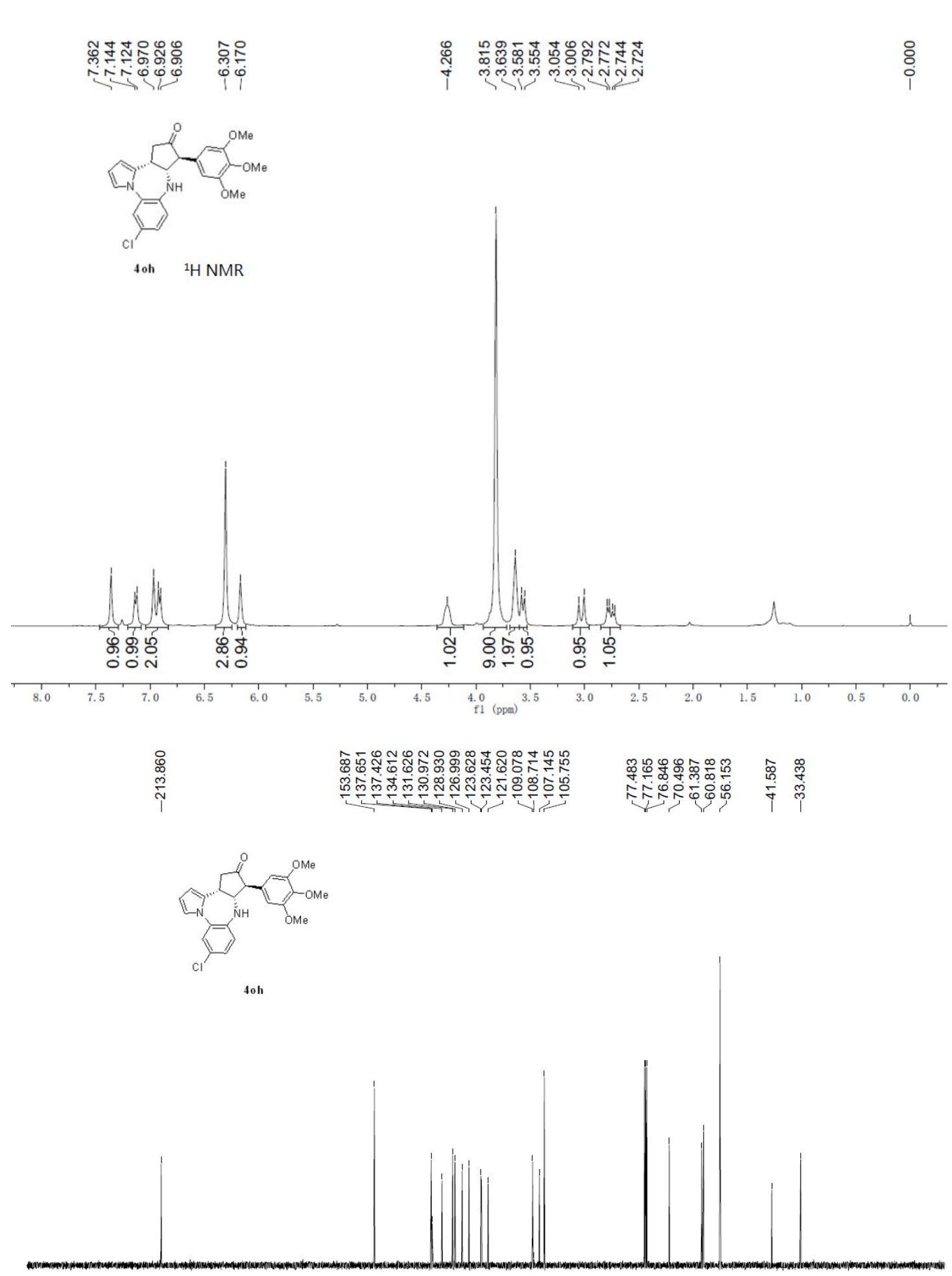

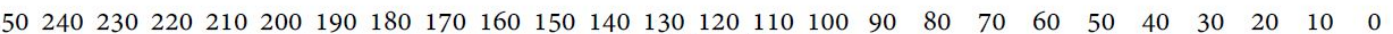
f1 (ppm) 


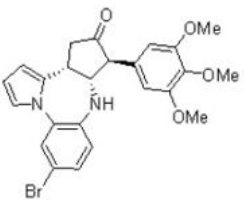

4oi ${ }^{1} \mathrm{H}$ NMR

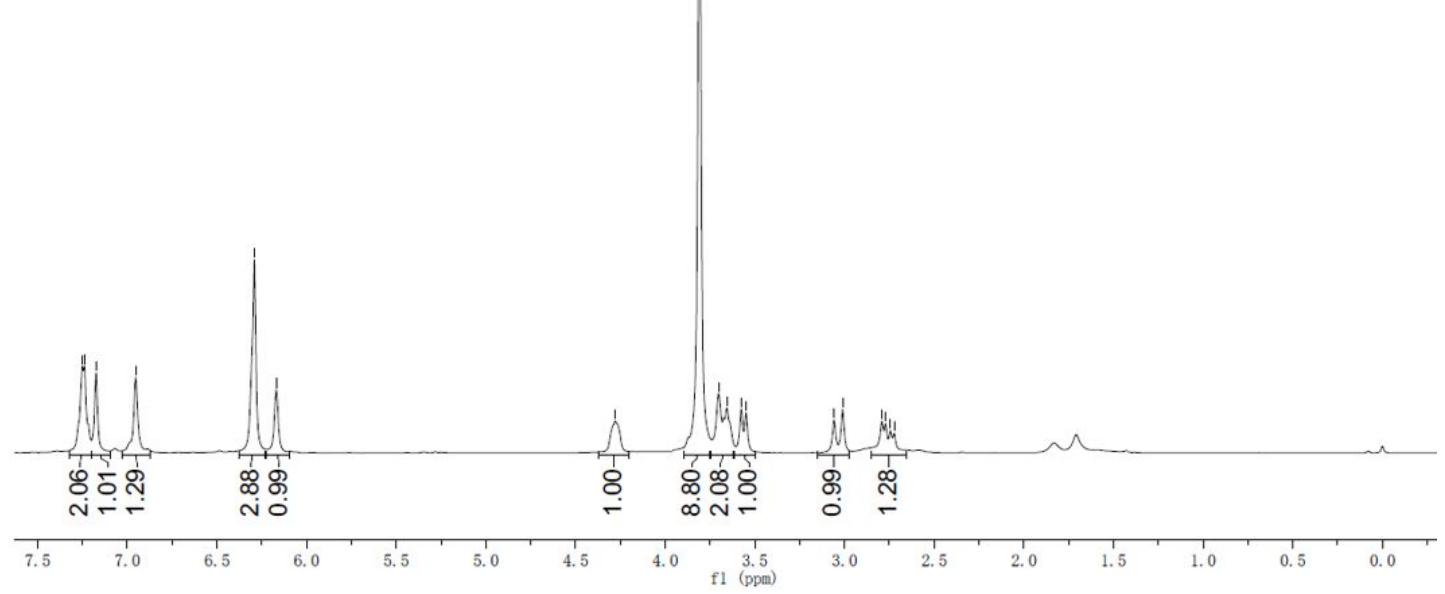

$\underset{\substack{N \\ i}}{\stackrel{0}{N}}$

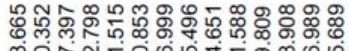
象守议

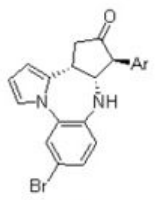

$40 \mathrm{i}$

${ }^{13} \mathrm{C}$ NMR

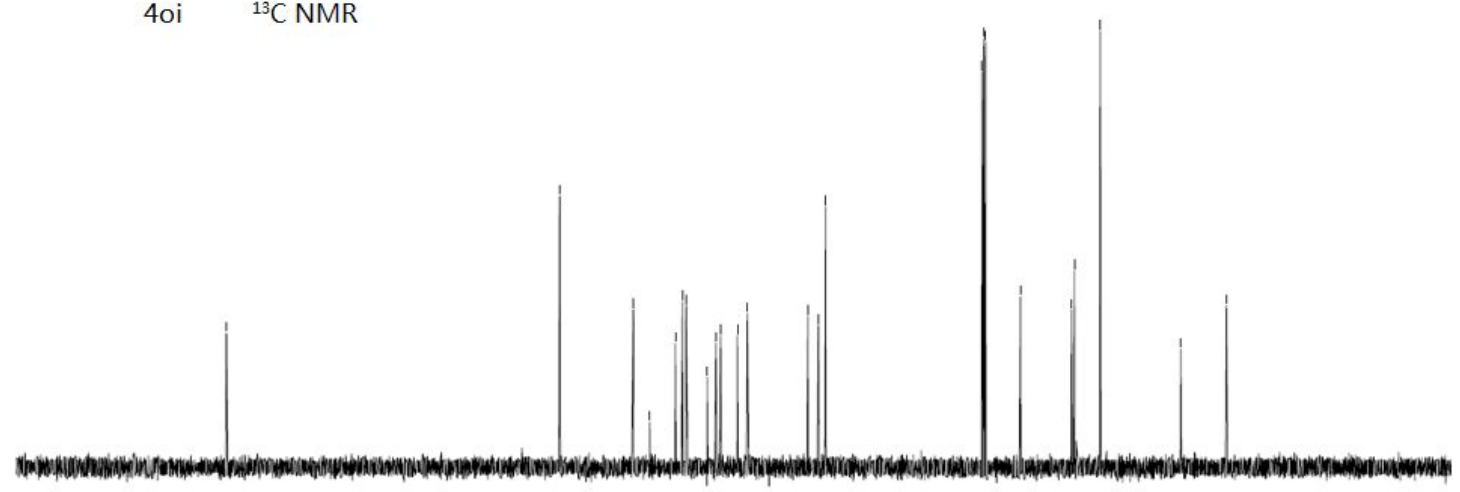

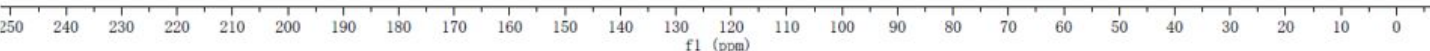




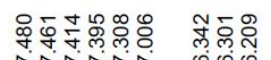

NiviN

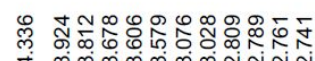

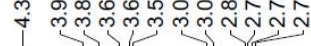

:

4 oj

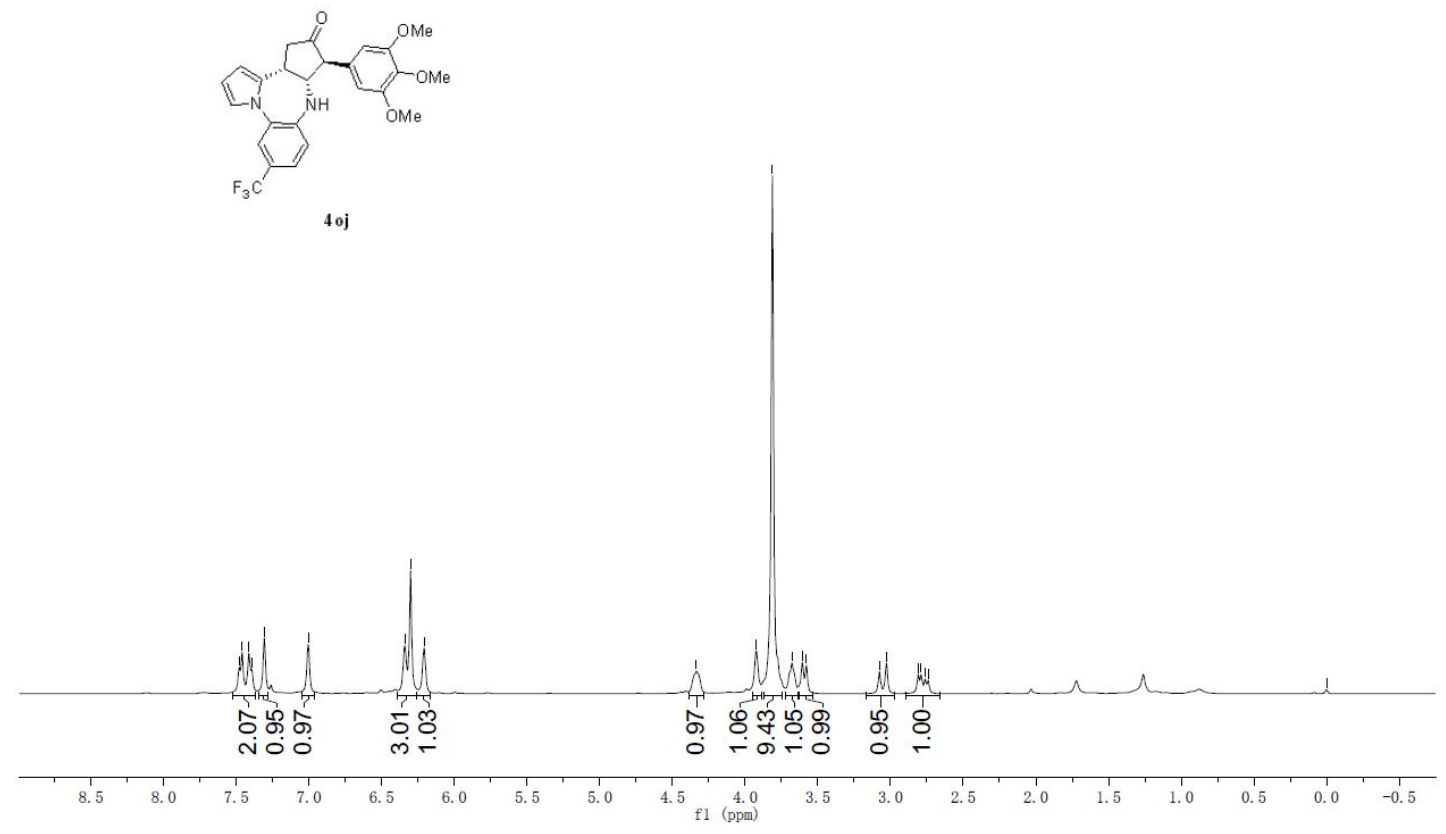

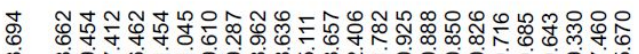

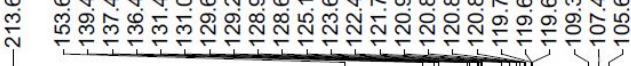

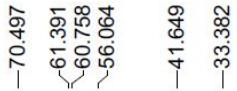

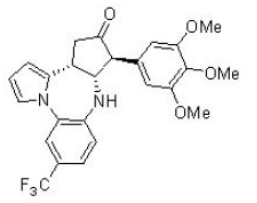

4 oj

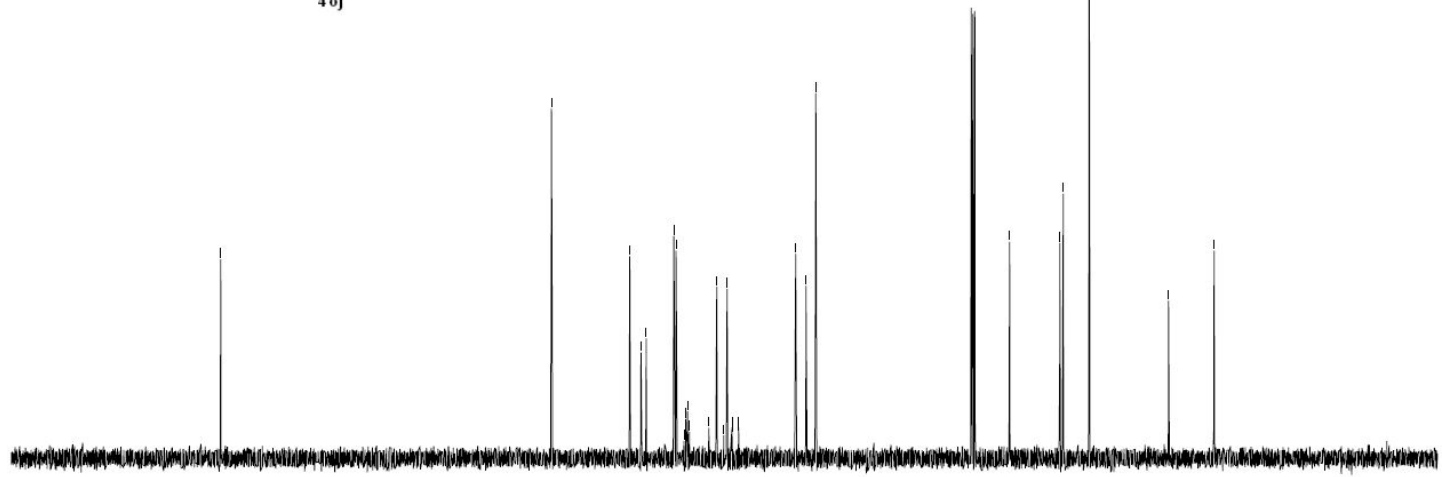

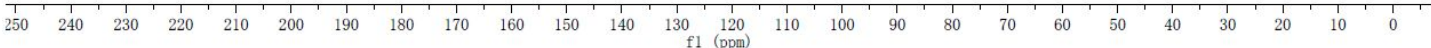




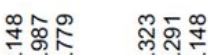

Tiऐ

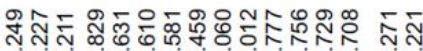

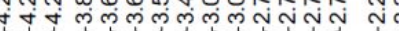

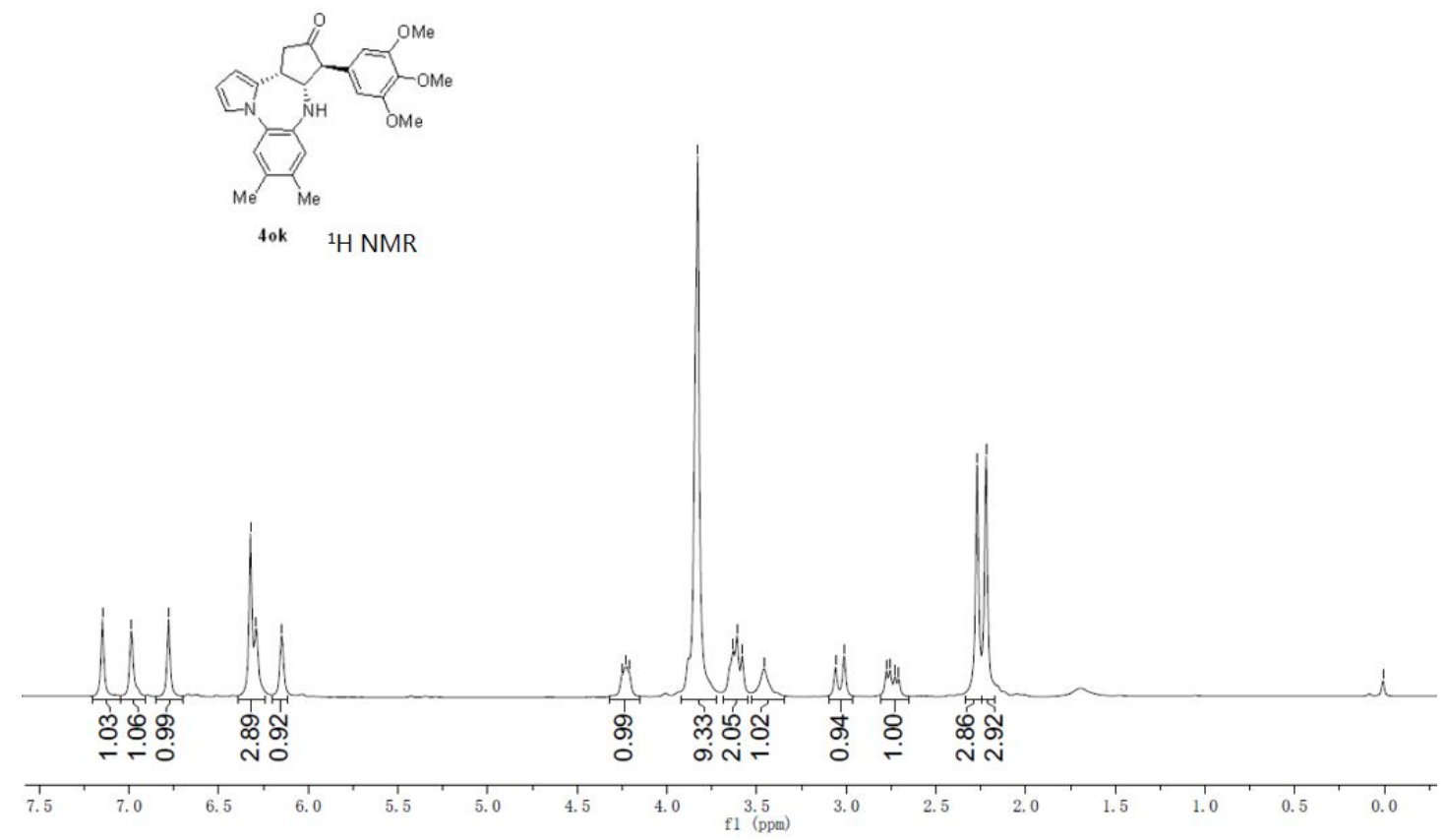

4ok 1 H NMR

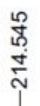

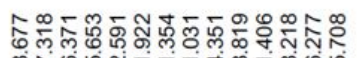

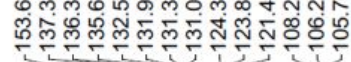

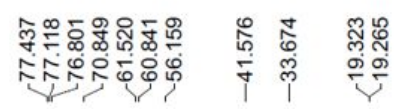

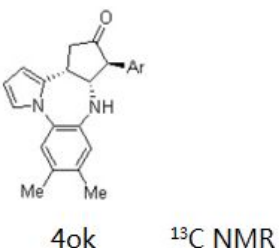

Jaw w.

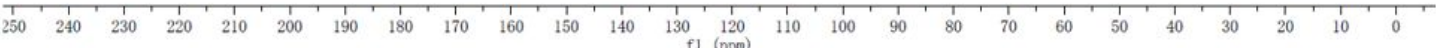


๒

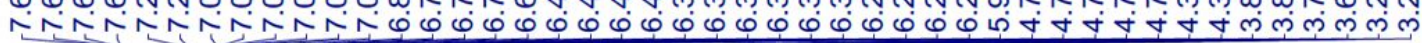
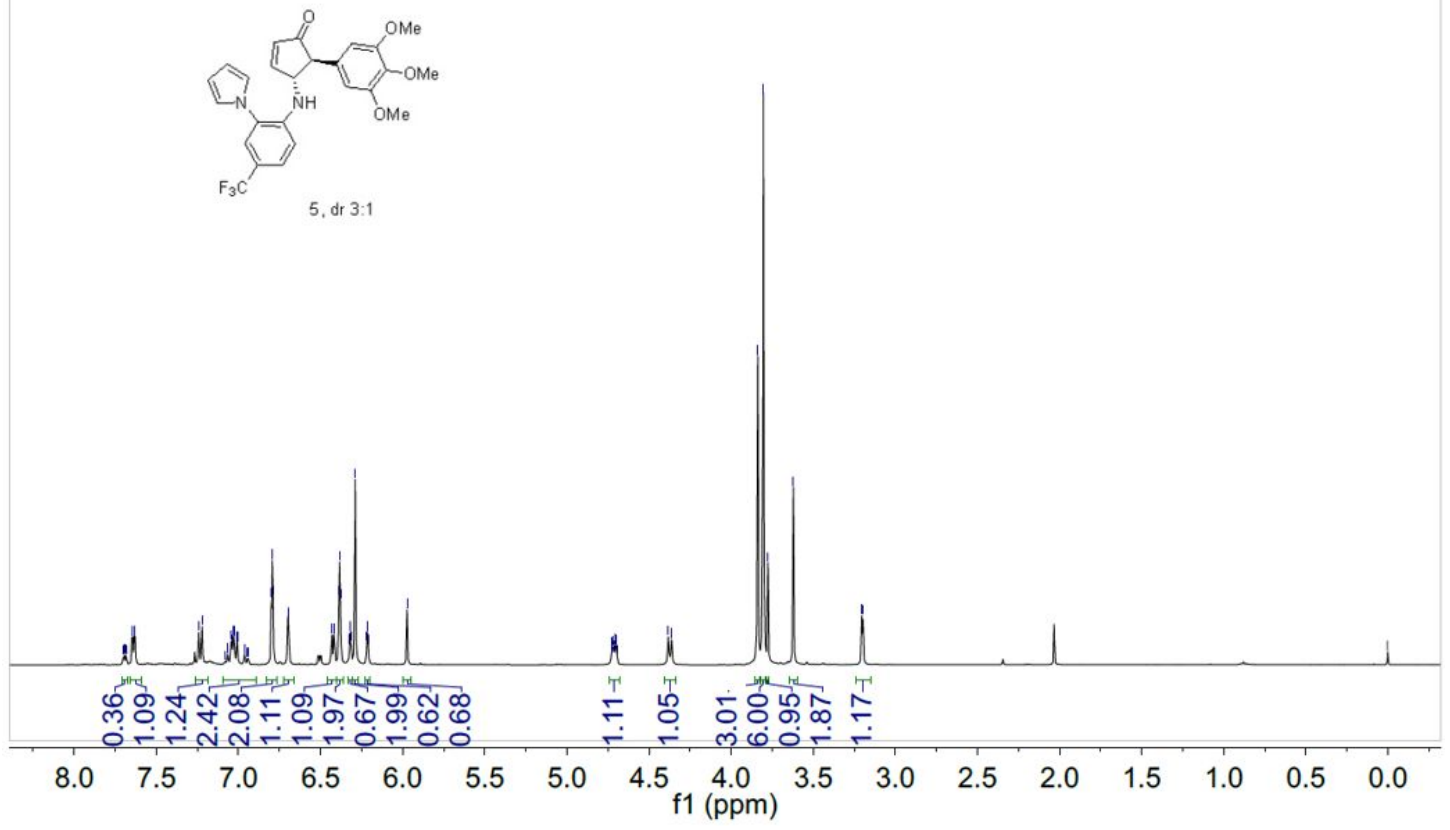

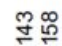

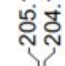

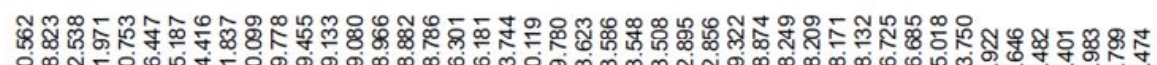

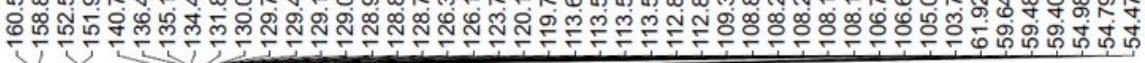

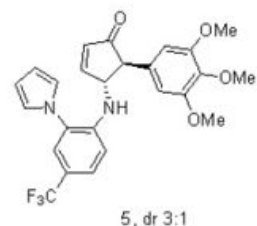

5, dr 3:1

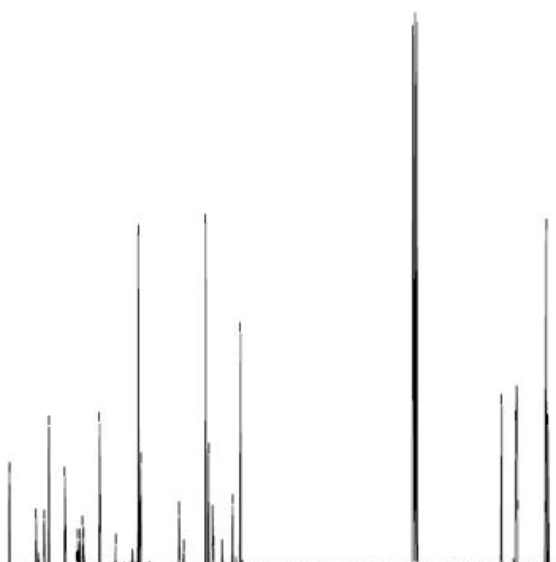

?10 $200190180170160150140130120 \begin{gathered}110 \begin{array}{c}100 \\ \mathrm{f} 1(\mathrm{ppm})\end{array} \\ 90\end{gathered}$ 


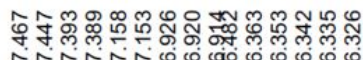
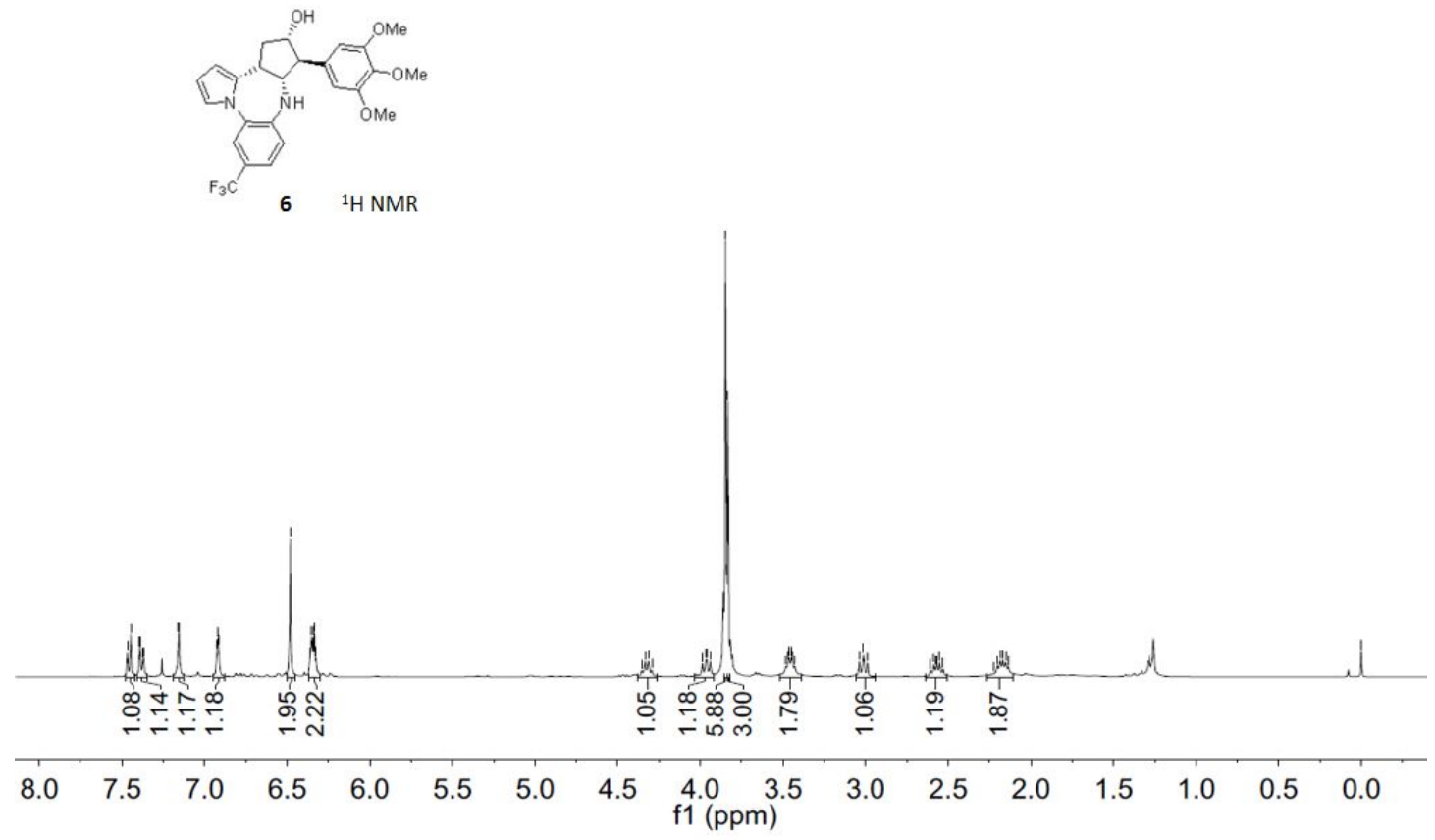

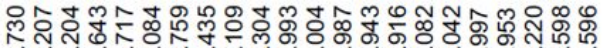

กำ

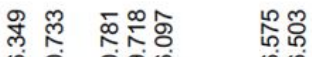

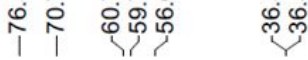

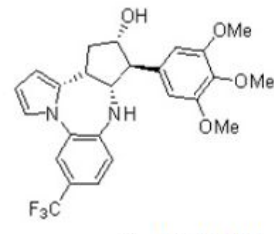

$6{ }^{13} \mathrm{C}$ NMR

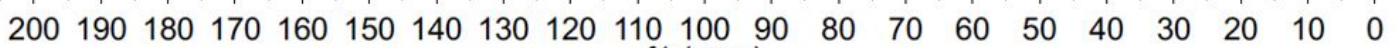
$\mathrm{f} 1(\mathrm{ppm})$ 


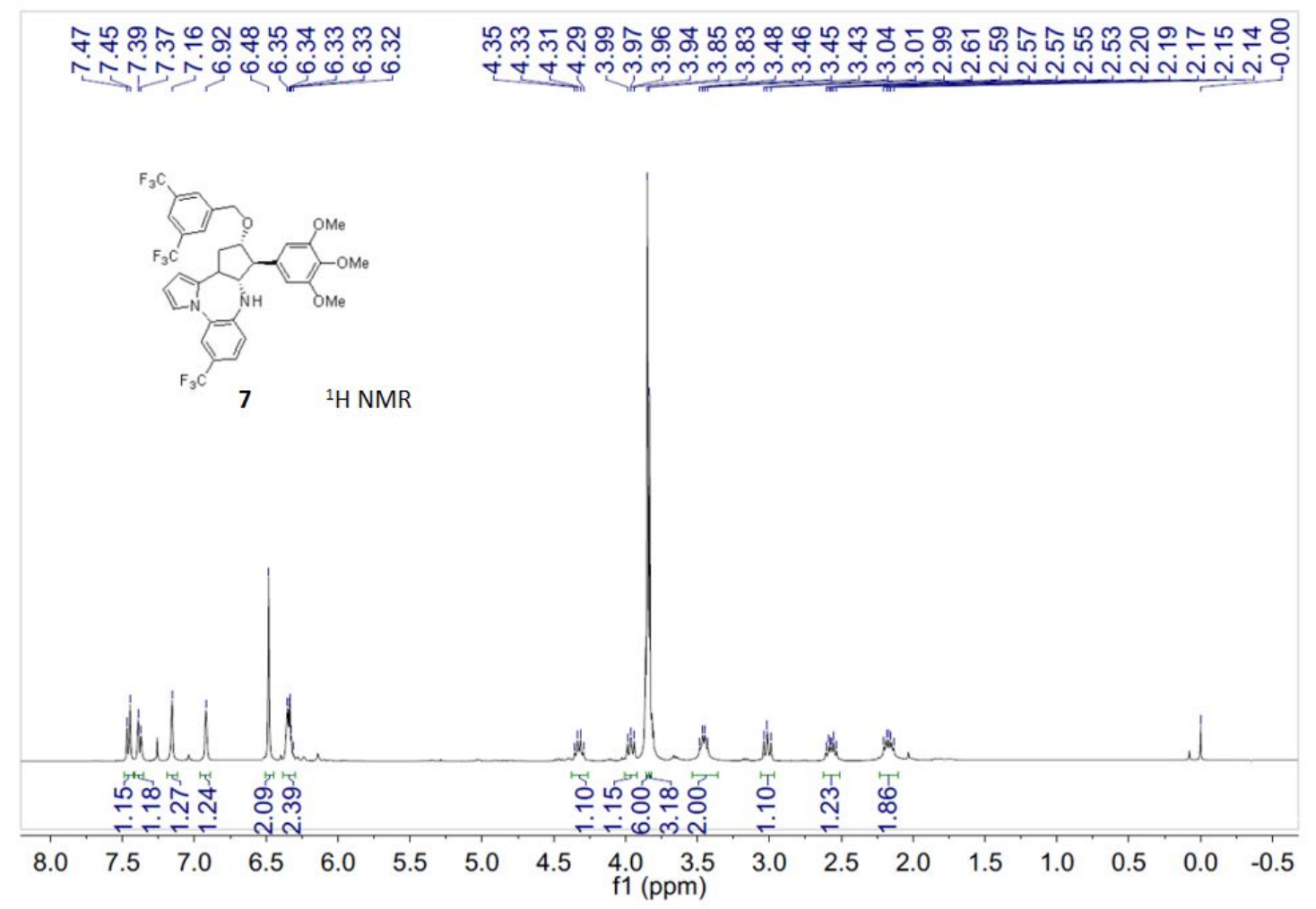

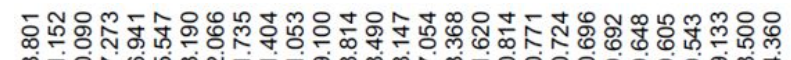

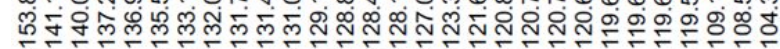
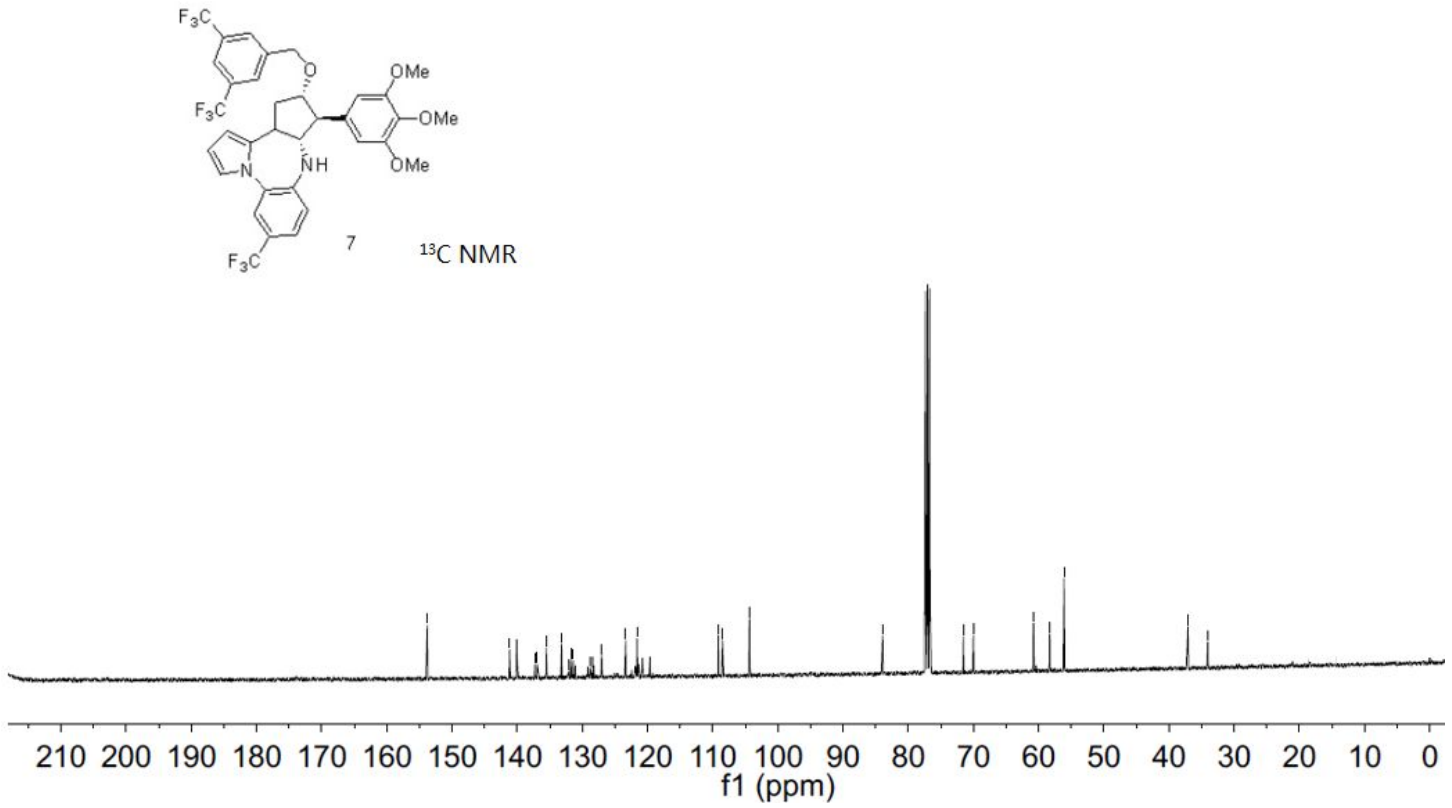


\section{HPLC analysis and spectra}

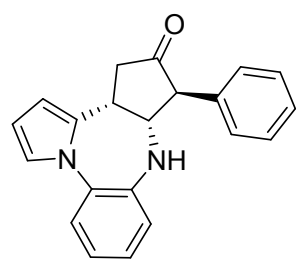

3aa, er 94:6

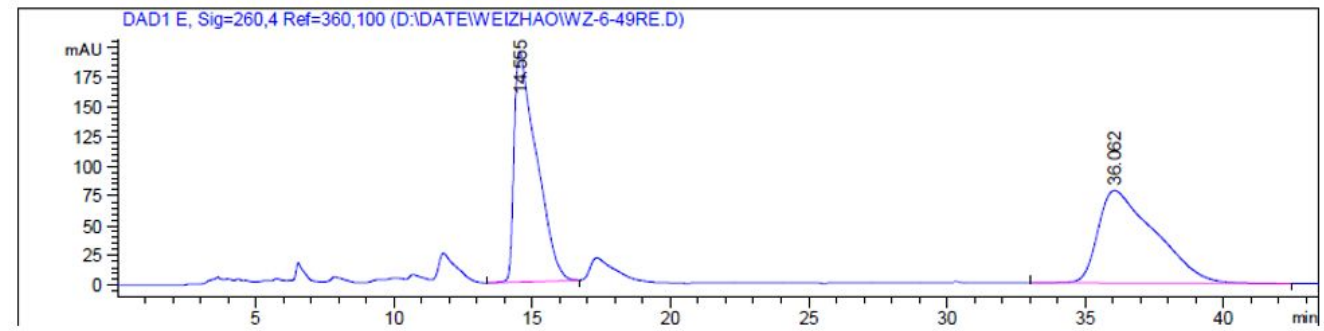

\begin{tabular}{|c|c|c|c|c|c|}
\hline$\#$ & {$[\mathrm{~min}]$} & {$[\mathrm{min}]$} & {$[\mathrm{mAU} * \mathrm{~s}]$} & [mAU] & $\%$ \\
\hline & & & & & \\
\hline 1 & $14.555 \mathrm{BB}$ & 0.7705 & $3.30861 e 4$ & 595.21759 & 49. \\
\hline 2 & $36.062 \mathrm{BB}$ & 1.9778 & $3.40767 e 4$ & 239.24181 & 50.7375 \\
\hline
\end{tabular}

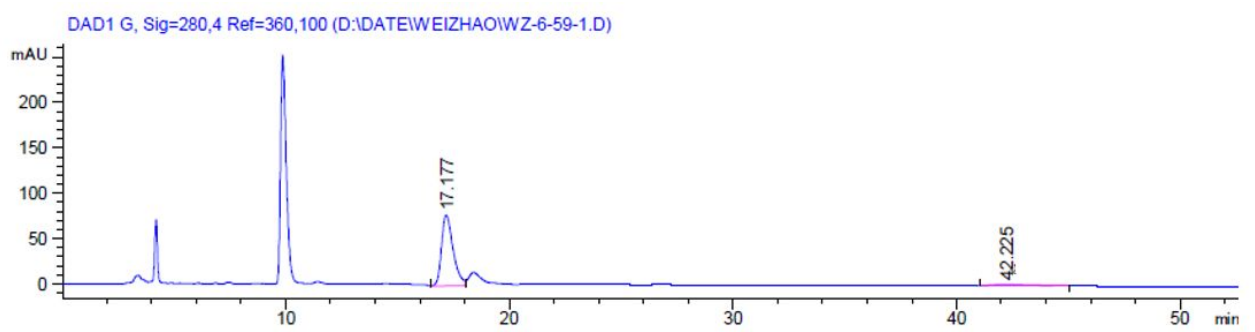

\begin{tabular}{|c|c|c|c|c|c|}
\hline \# & {$[\mathrm{min}]$} & {$[\mathrm{min}]$} & {$[\mathrm{mAU} * \mathrm{~s}]$} & {$[\mathrm{mAU}]$} & $\%$ \\
\hline & & & & & \\
\hline 1 & $17.177 \mathrm{BV}$ & 0.5458 & 2796.78906 & 78.10120 & \\
\hline 2 & $42.225 \mathrm{MM}$ & 1.8875 & 173.93825 & 1.53591 & 5.8551 \\
\hline
\end{tabular}




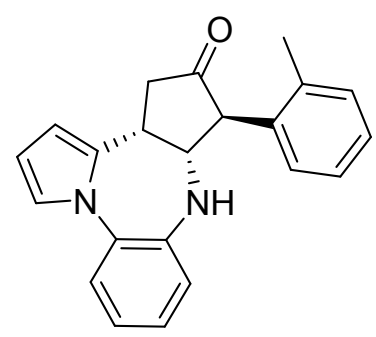

3ba, er 90.5:9.5
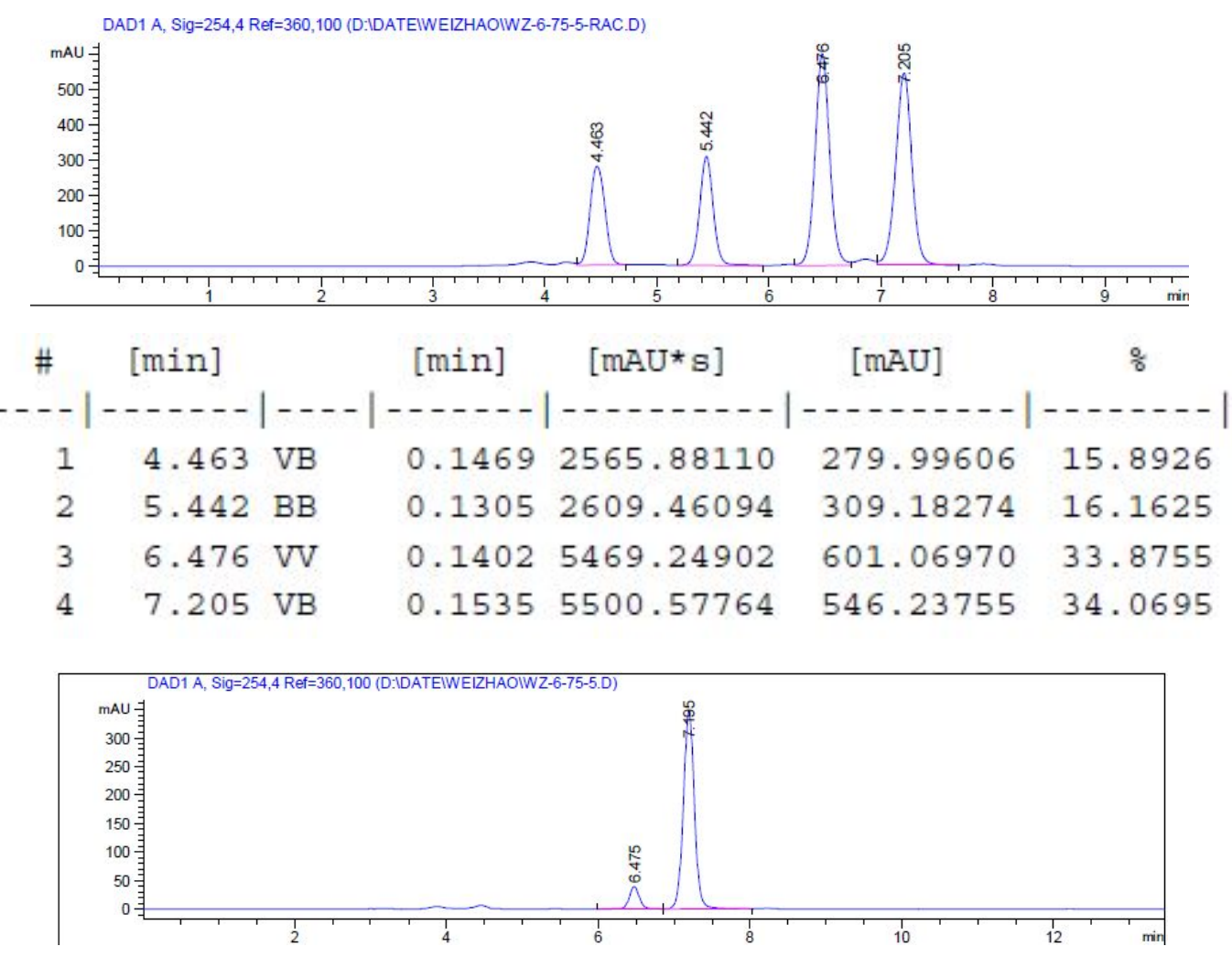

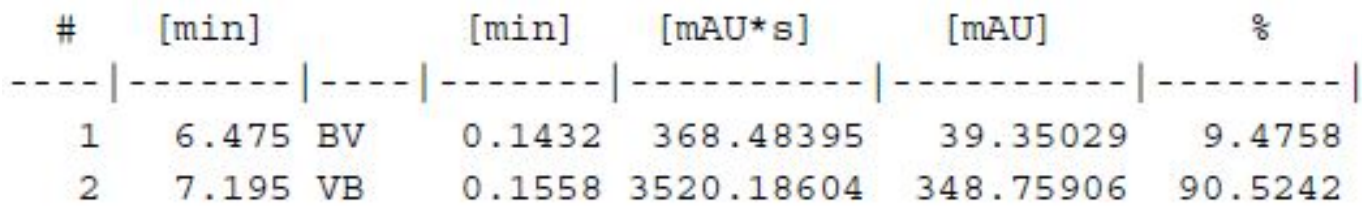




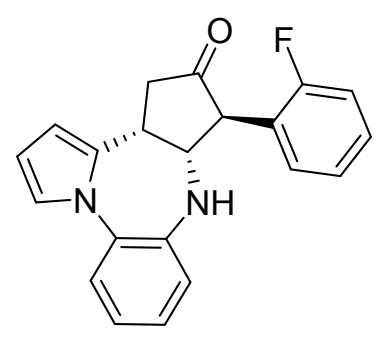

3ca, er 93:7

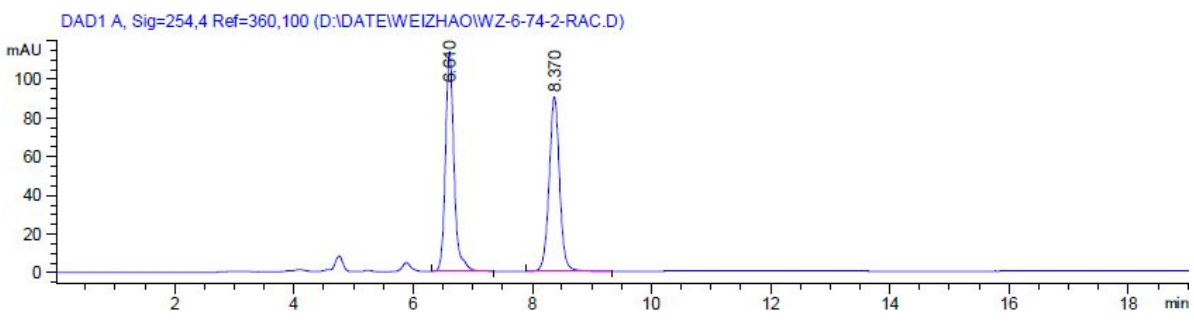

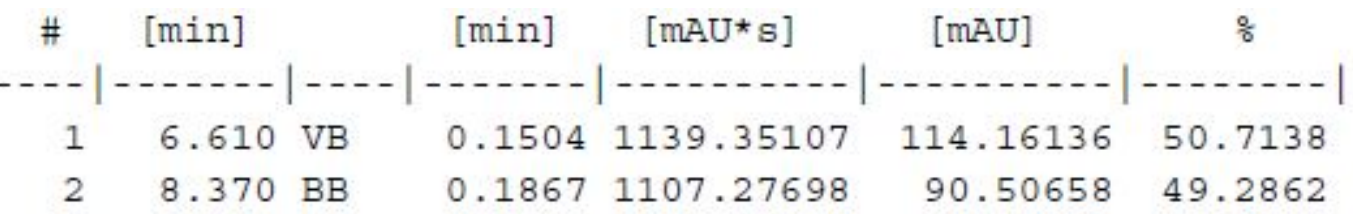

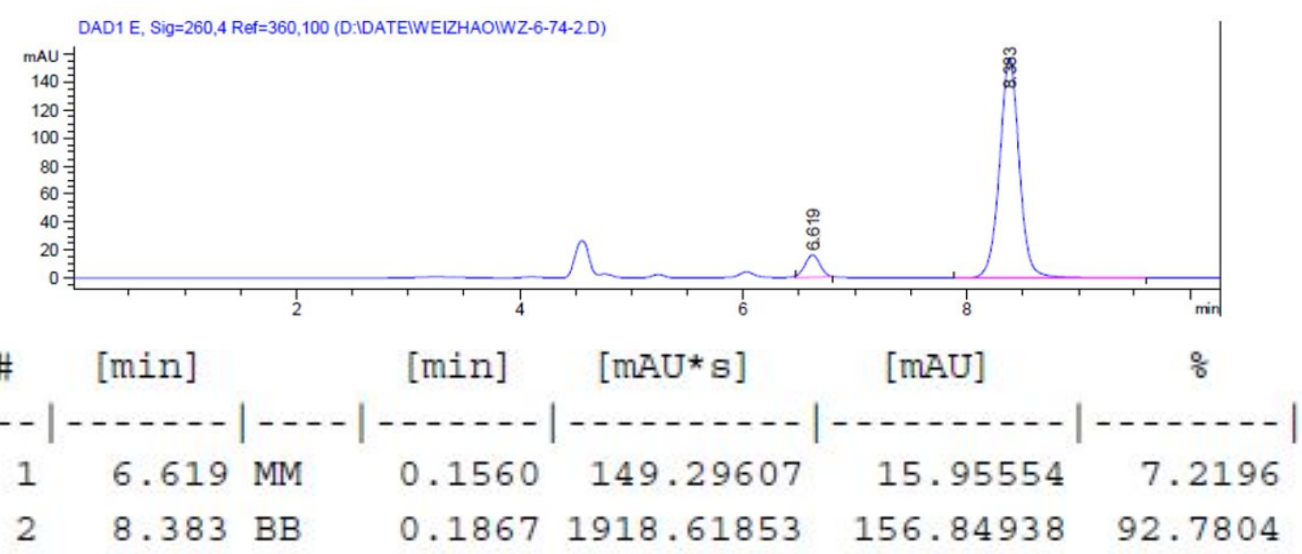




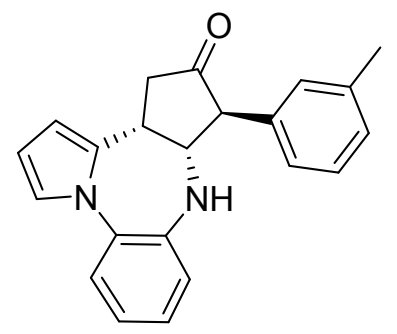

3da, er 92:8
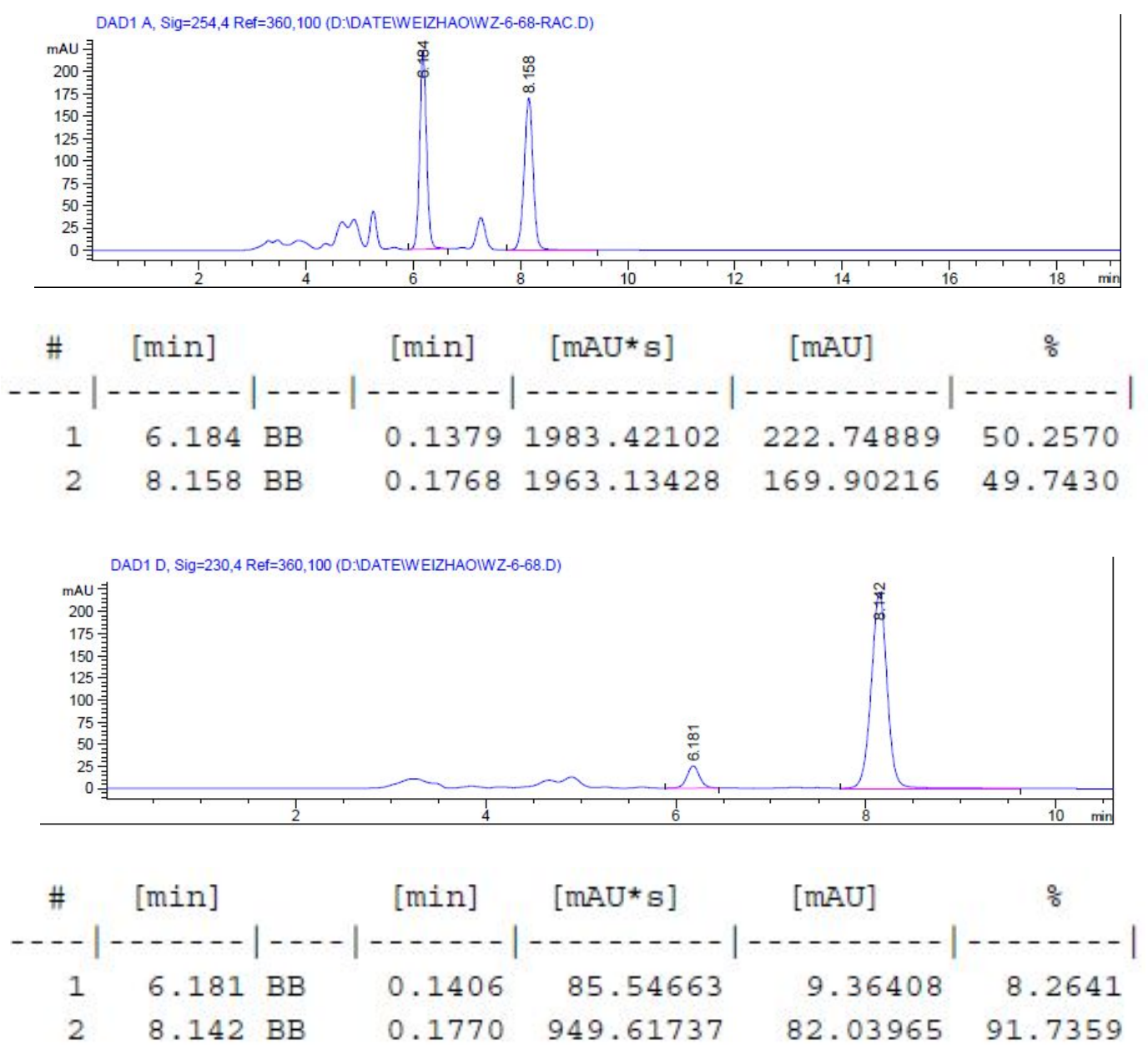


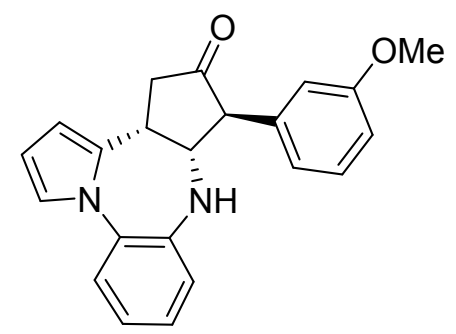

3ea, er 95.5:4.5
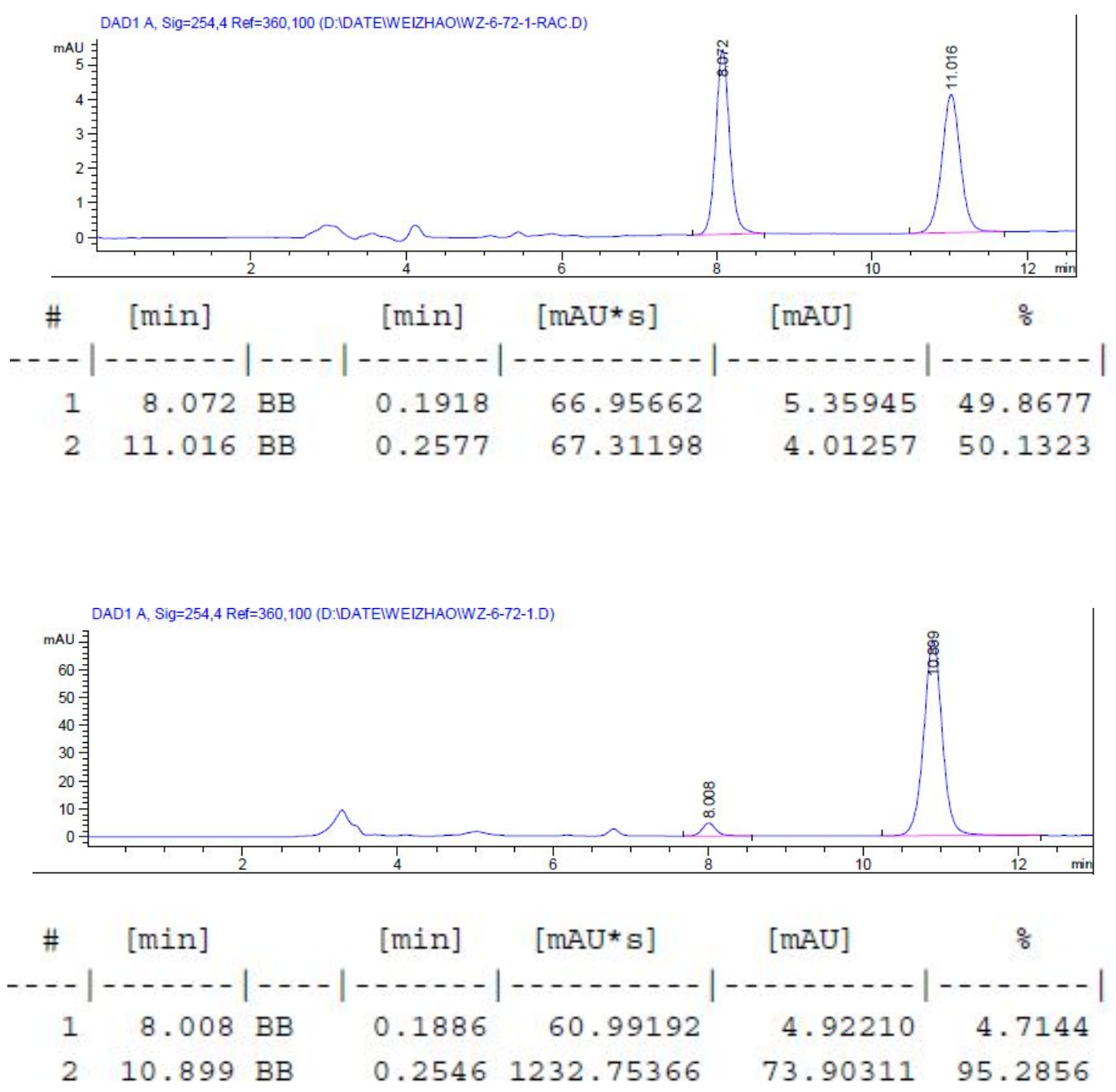


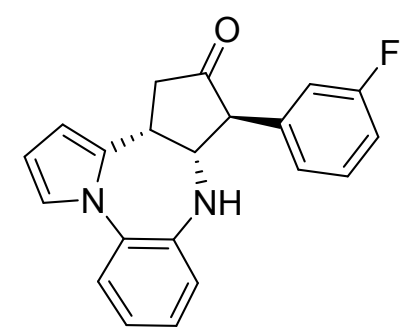

3fa, er 93.5:6.5
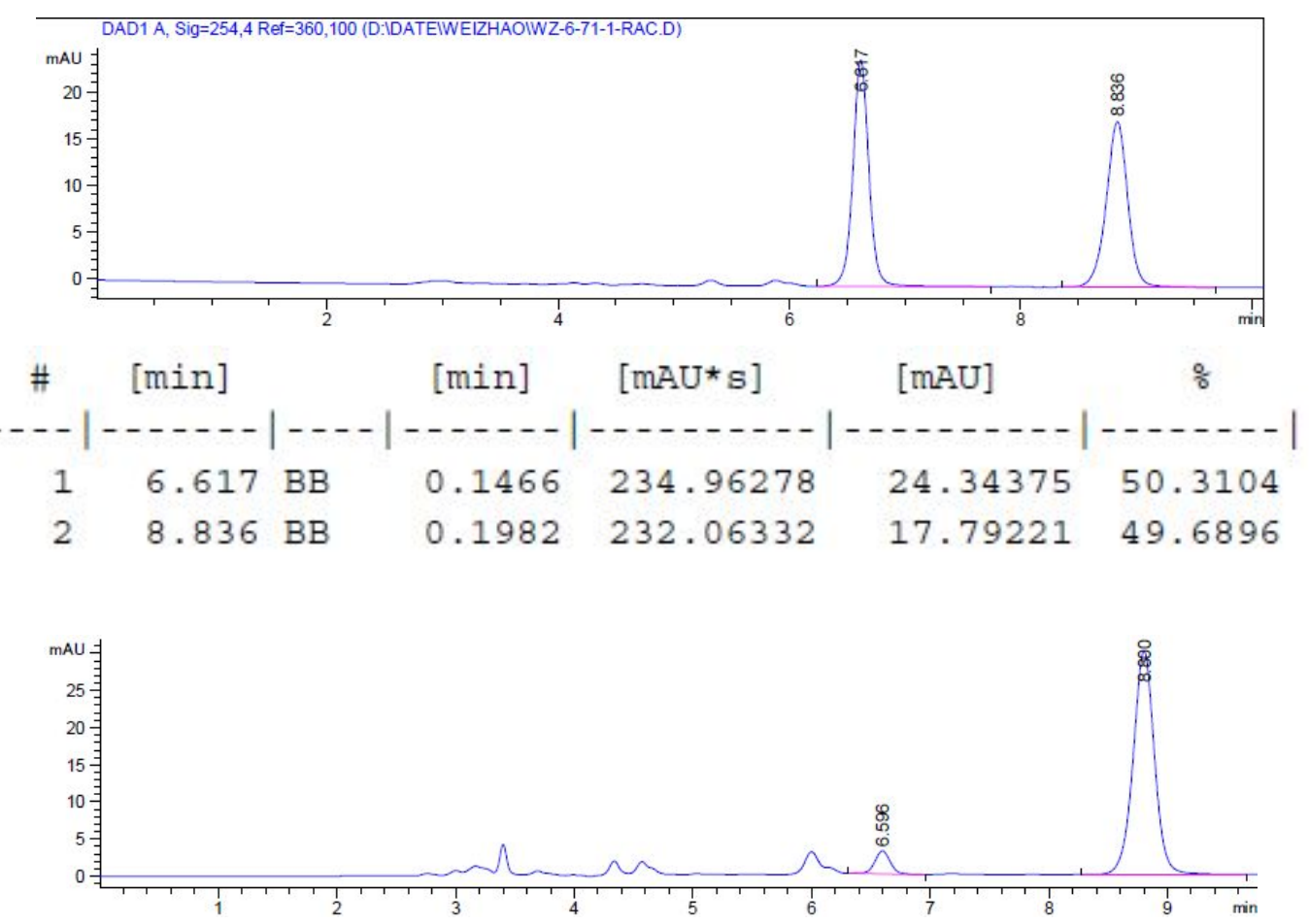

\begin{tabular}{|c|c|c|c|c|c|c|}
\hline$\#$ & [min] & & {$[\mathrm{min}]$} & {$[\mathrm{mAU} * \mathrm{~s}]$} & [mAU] & \% \\
\hline & & & & & & \\
\hline 1 & 6.596 & BB & 0.1316 & 13.61609 & 1.59498 & 6.3717 \\
\hline 2 & 8.800 & BB & 0.1925 & 200.07965 & 15.93365 & 93.6283 \\
\hline
\end{tabular}




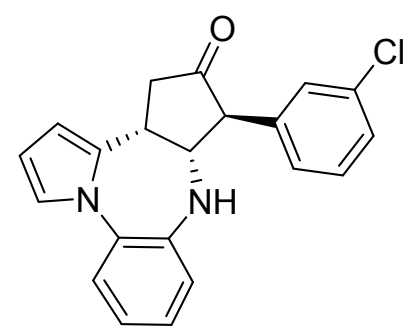

3ga, er 93:7
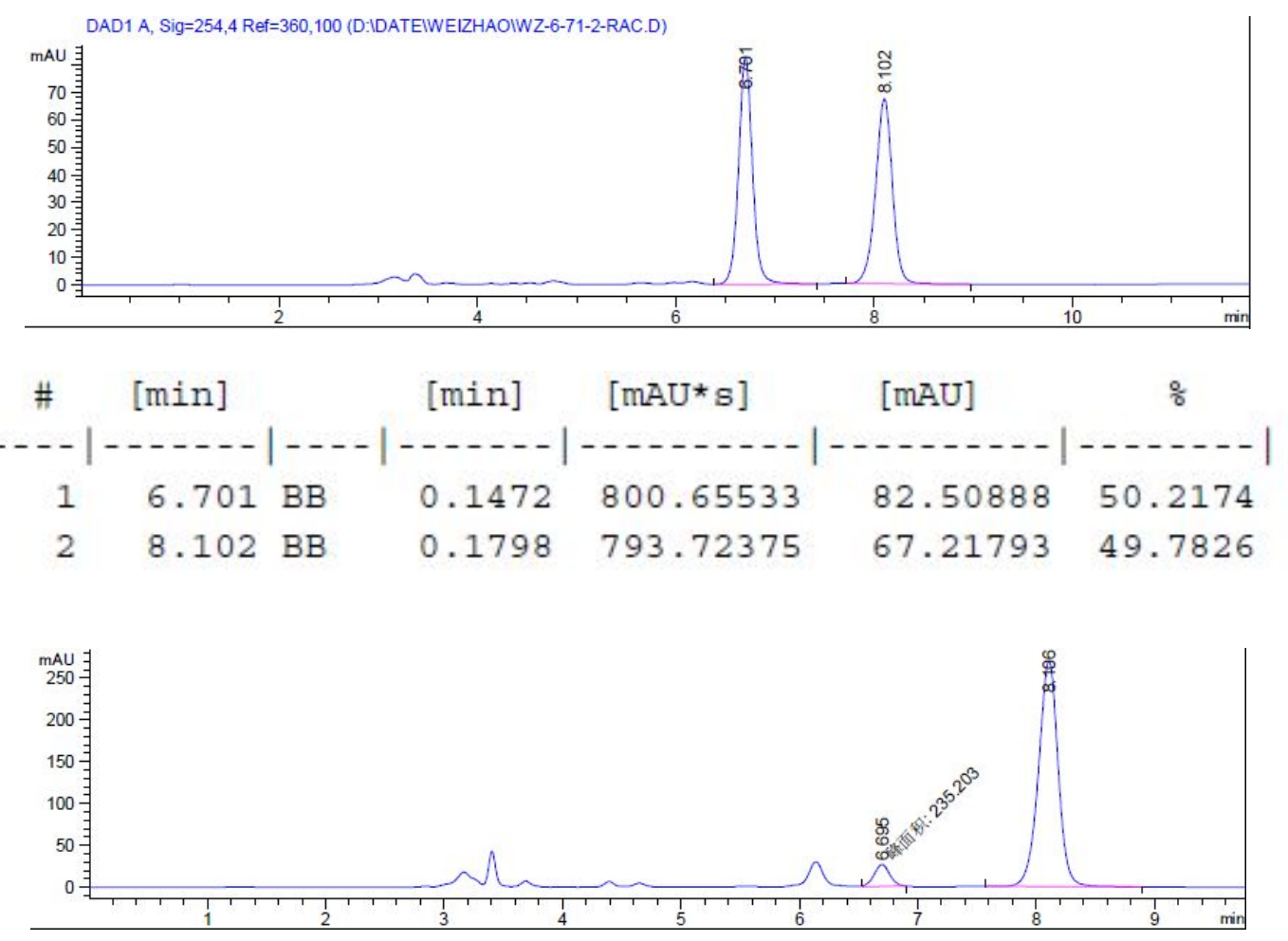

\begin{tabular}{|c|c|c|c|c|c|c|}
\hline \# & {$[\mathrm{min}]$} & & {$[\mathrm{min}]$} & {$\left[\mathrm{mAU}{ }^{*} \mathrm{~s}\right]$} & [mAU] & $\%$ \\
\hline & & & & 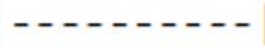 & 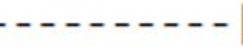 & $-\cdots-\cdots$ \\
\hline 1 & 6.695 & MM & 0.1514 & 235.20337 & 25.88550 & 7.0344 \\
\hline 2 & 8.106 & BB & 0.1766 & 3108.40137 & 269.44336 & 92.9656 \\
\hline
\end{tabular}




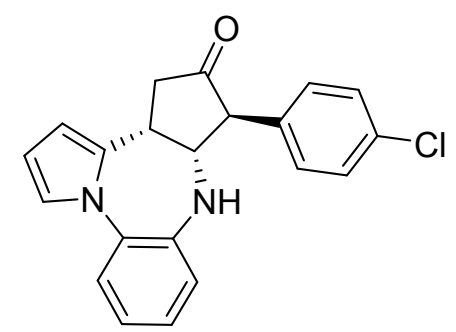

3ha, er 88:12
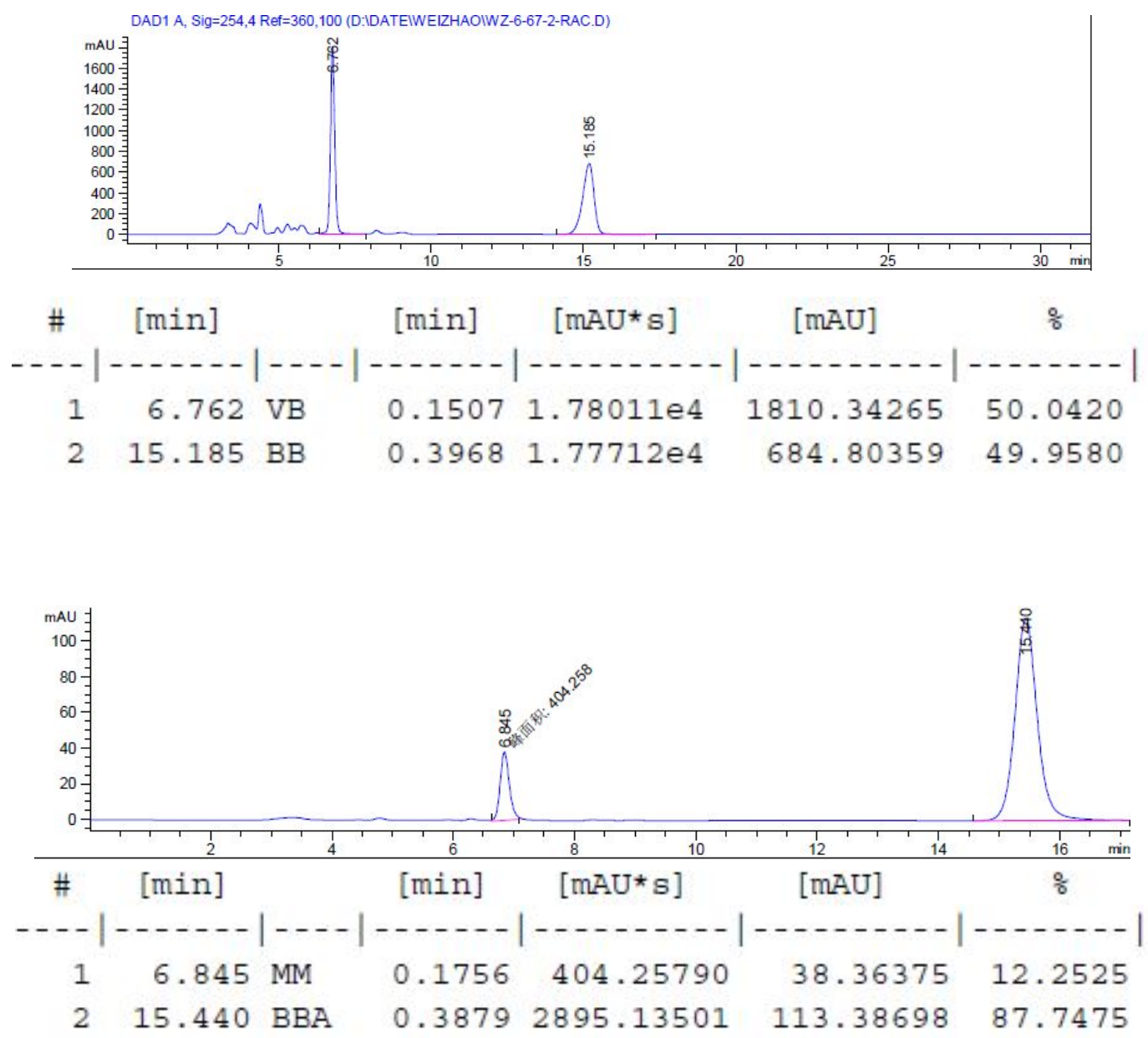


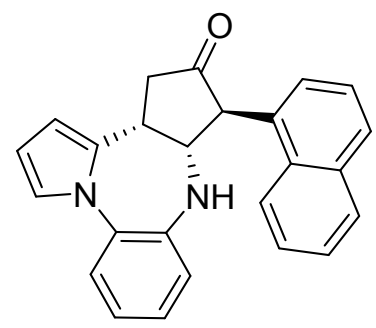

3ia, er 91:9
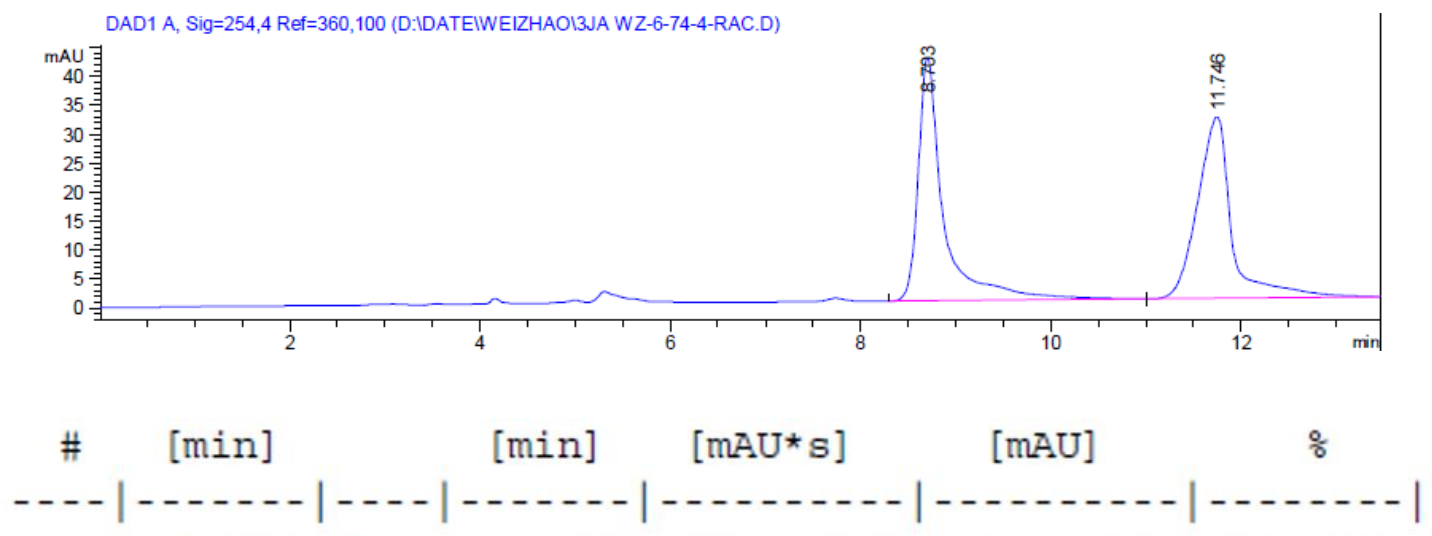
$18.703 \mathrm{BB}$
$0.2672 \quad 789.63995$
$42.02449 \quad 50.0505$
$211.746 \mathrm{BBA}$
$0.3767 \quad 788.04590$
$31.40674 \quad 49.9495$

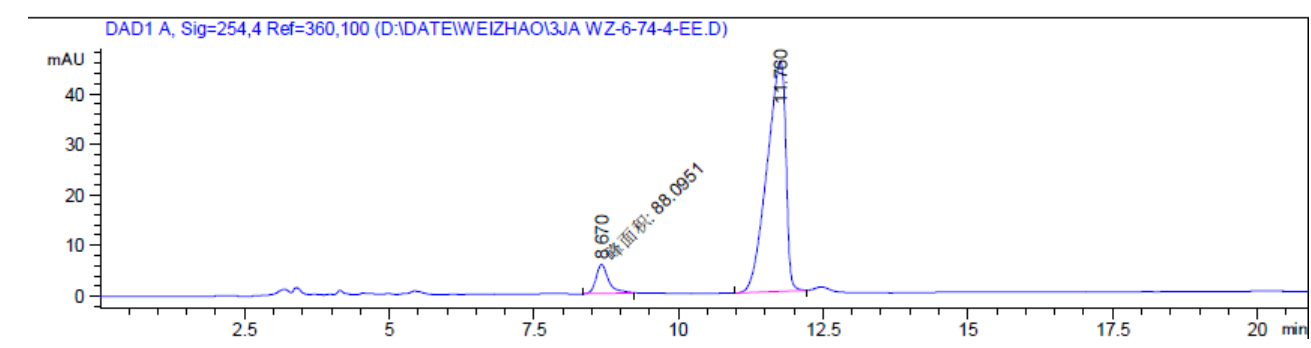

\begin{tabular}{|c|c|c|c|c|c|c|}
\hline$\#$ & [min] & & [min] & {$[\mathrm{mAU} * \mathrm{~s}]$} & [mAU] & $\%$ \\
\hline & & & 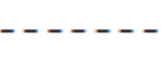 & -- & $\cdots-\cdots$ & - \\
\hline 1 & 8.670 & MM & 0.2546 & 88.09514 & 5.76687 & 8974 \\
\hline 2 & 11.760 & $\mathrm{BB}$ & 0.3468 & 1027.40613 & 45.60326 & 92.1026 \\
\hline
\end{tabular}




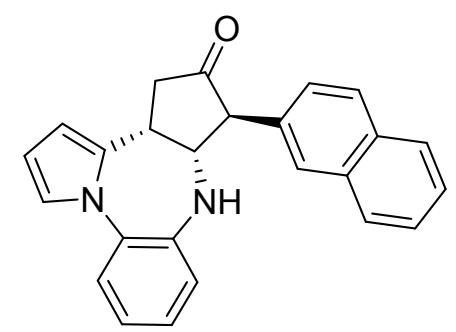

3ja, er 92.5:7.5
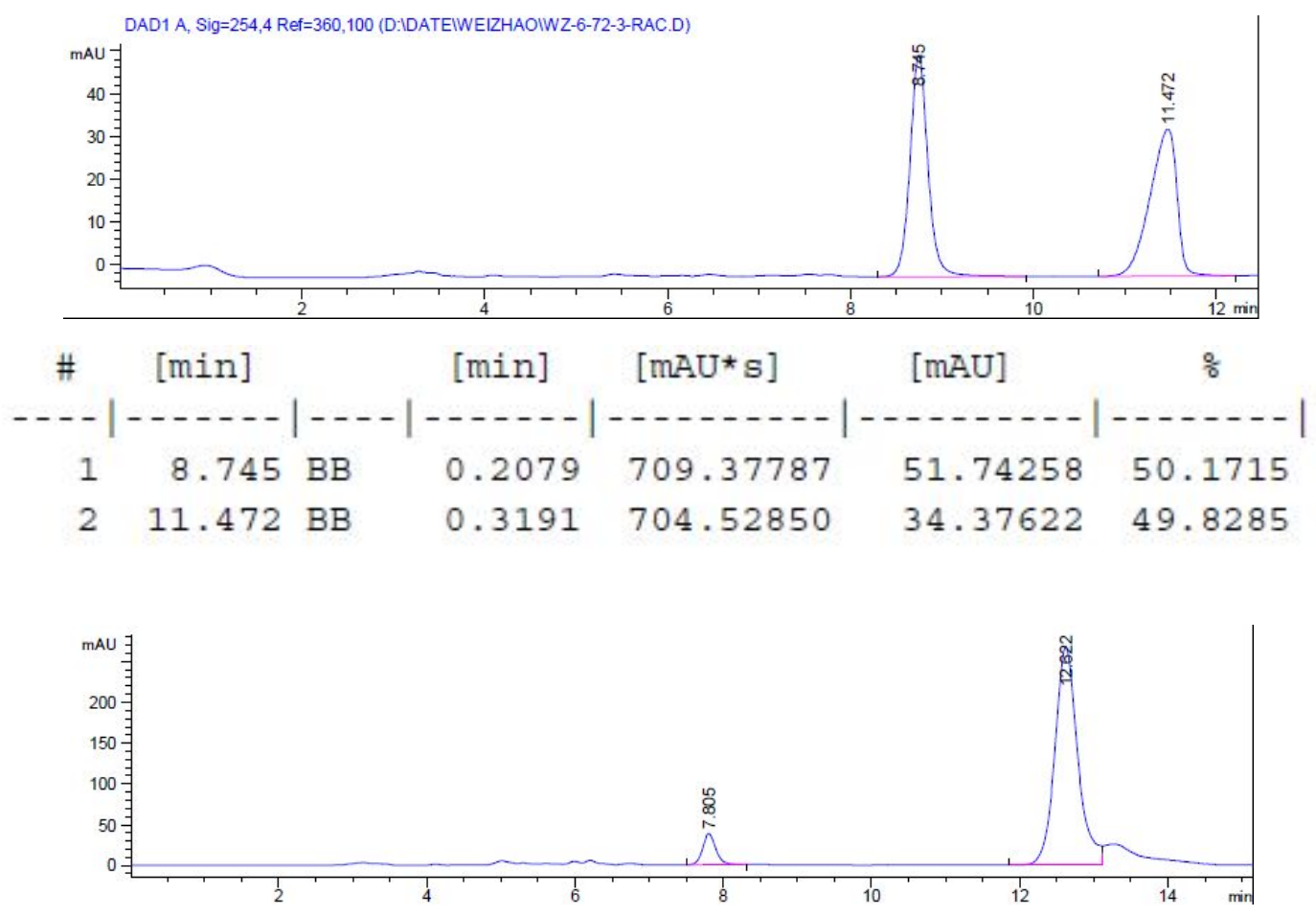

\begin{tabular}{|c|c|c|c|c|c|c|}
\hline \# & {$[\mathrm{min}]$} & & [min] & {$[\mathrm{mAU} * \mathrm{~s}]$} & [mAU] & $\%$ \\
\hline & & & & -- & $\ldots$ & -- \\
\hline 1 & 7.805 & $B B$ & 0.1878 & 468.91104 & 38.03207 & 7.5039 \\
\hline 2 & 12.622 & BV & 0.3233 & 5779.98242 & 268.32535 & 92.4961 \\
\hline
\end{tabular}




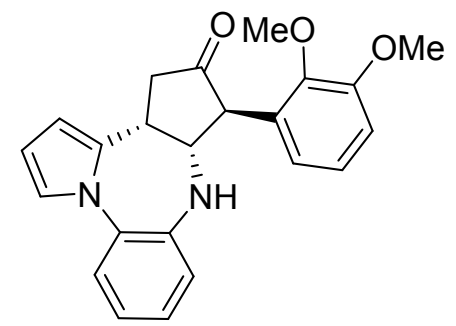

3ka, er 96:4
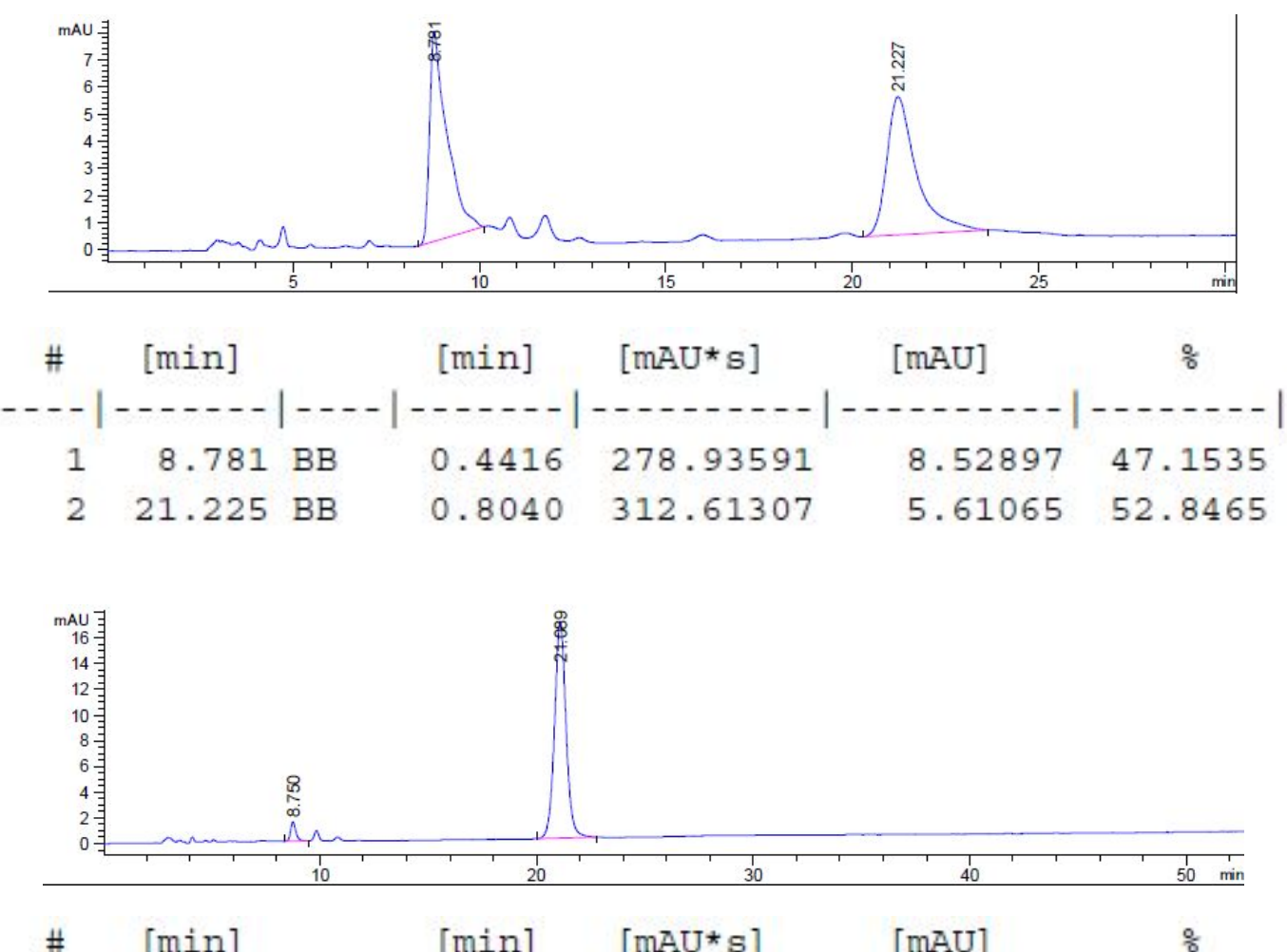

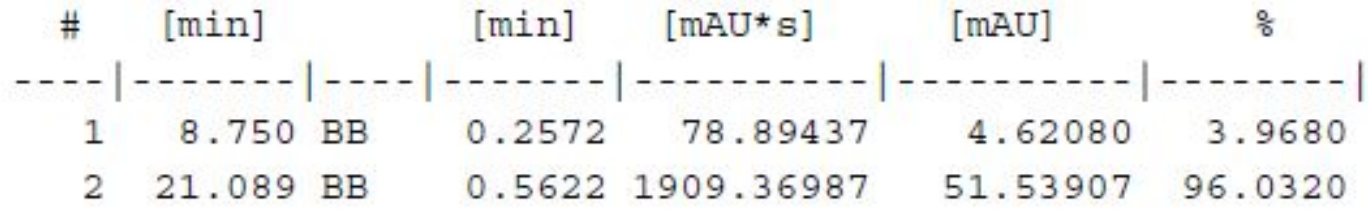




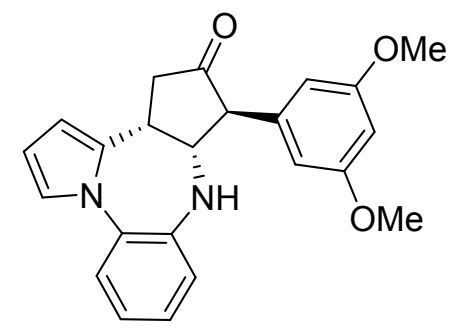

3la, er 96:4
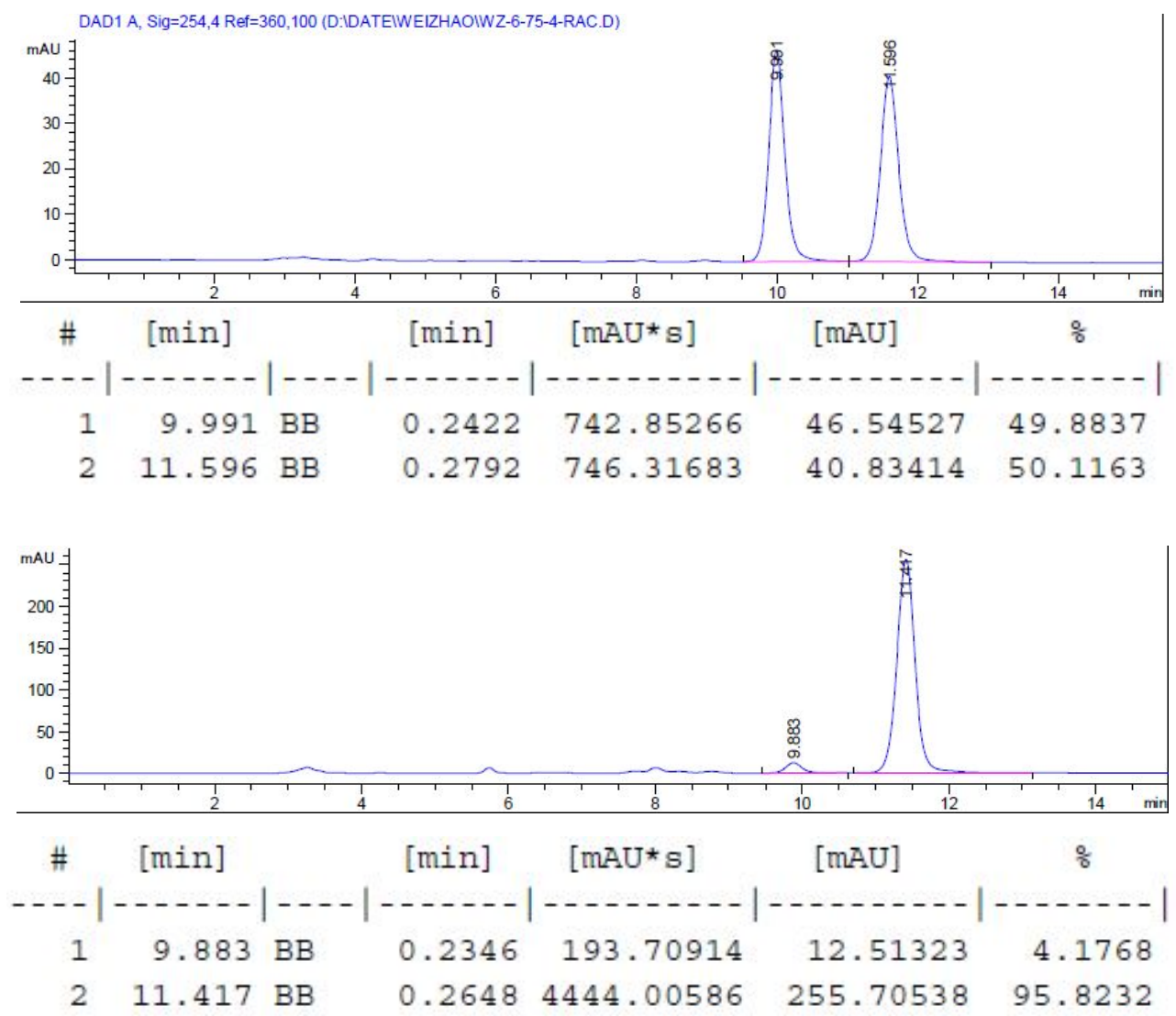


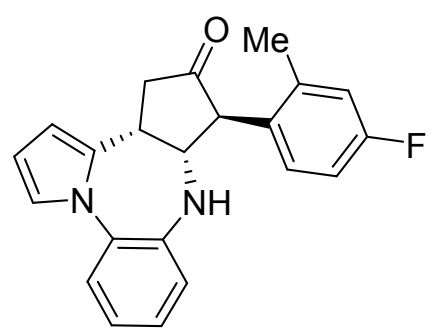

3ma, er 92:8
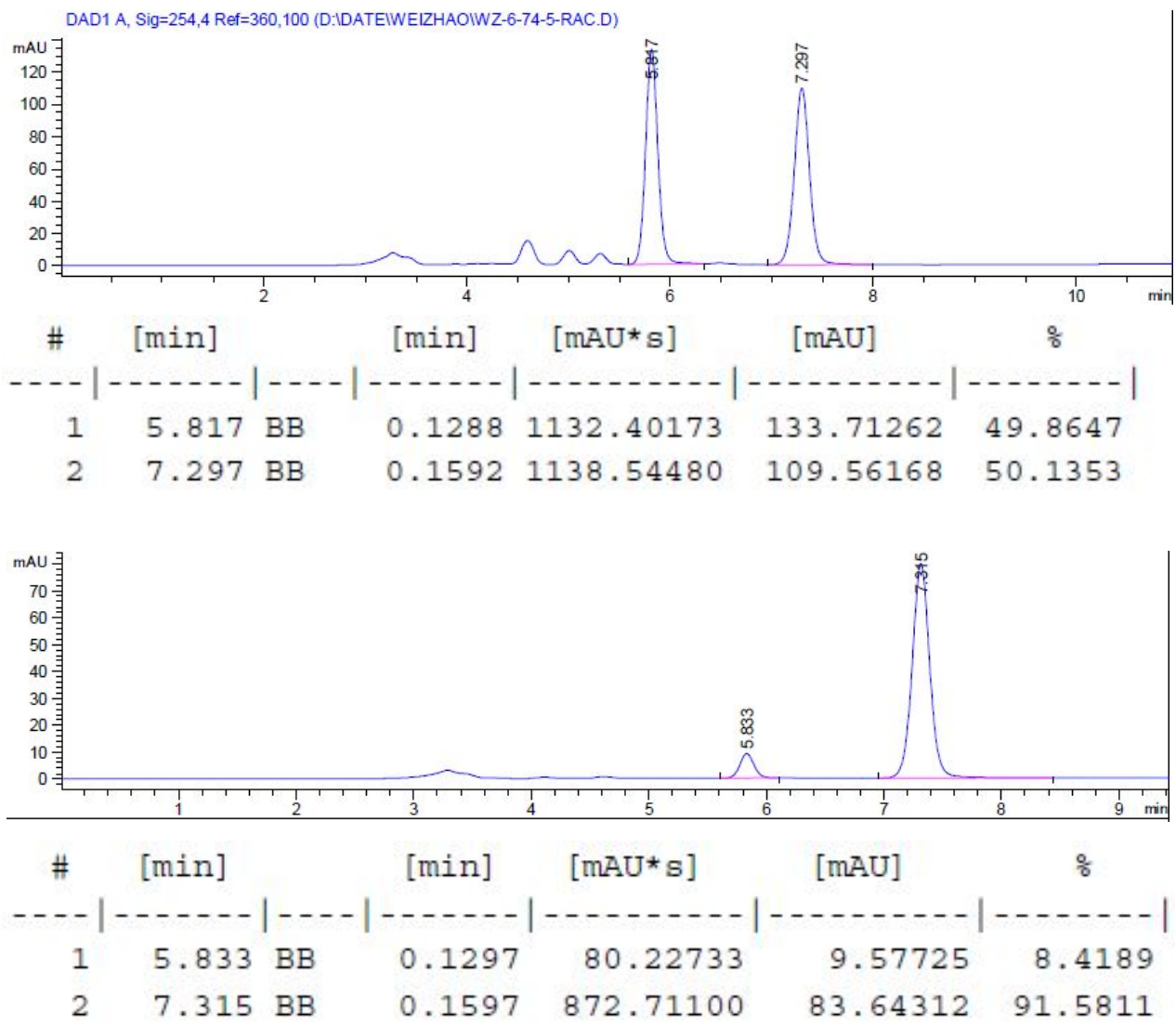


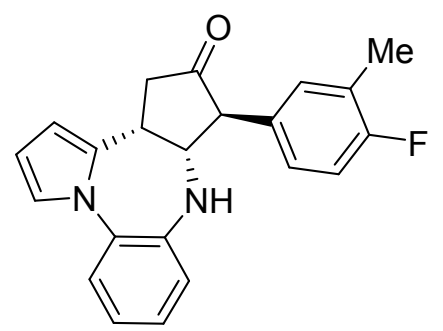

3na, er 93:7
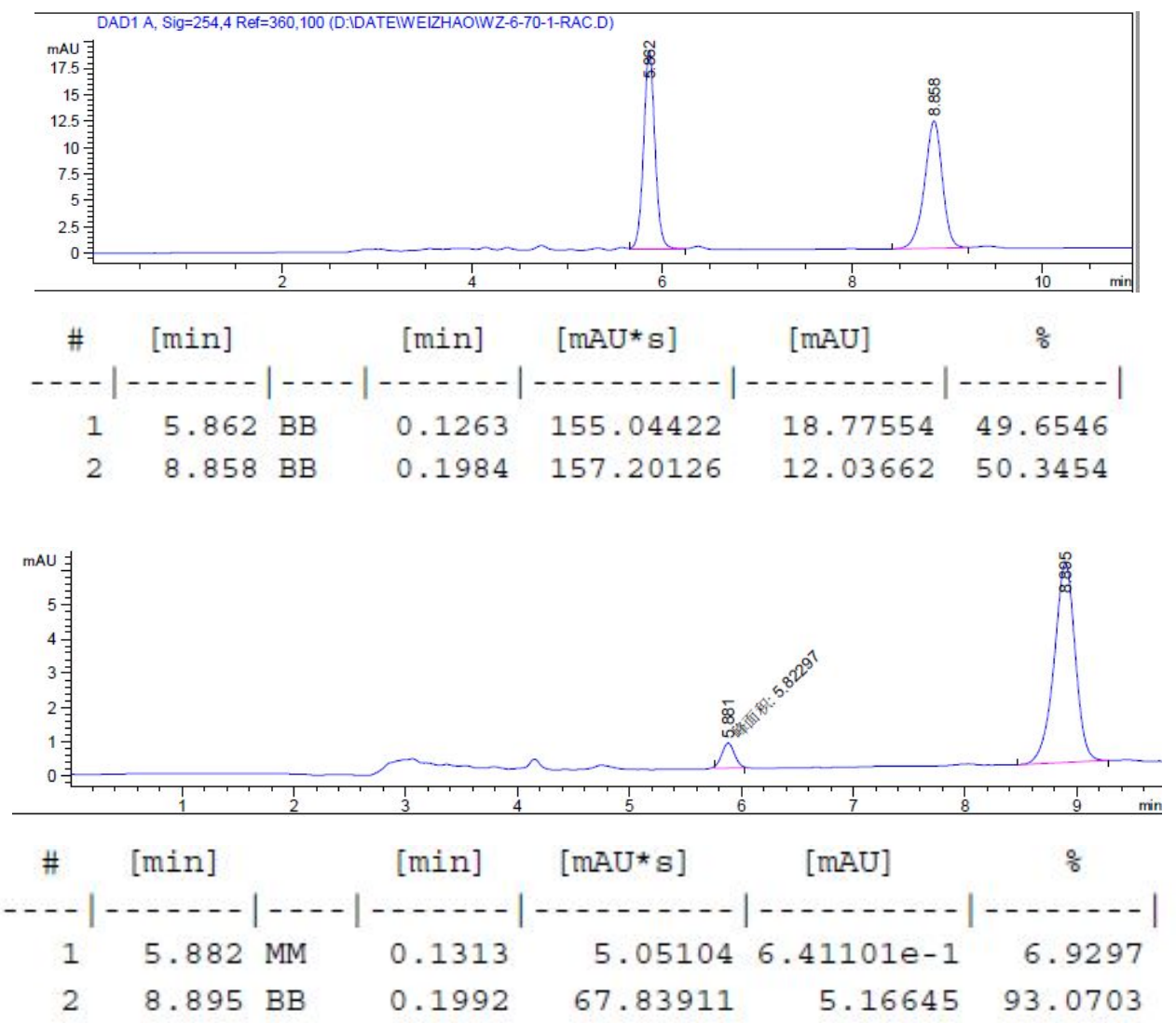


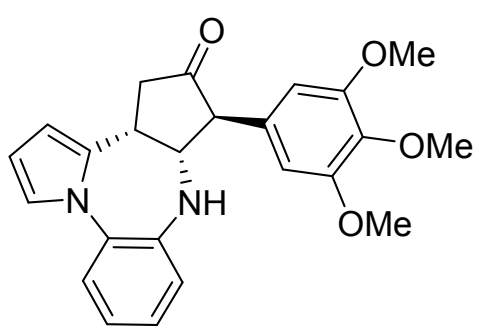

3oa, er 98.5:1.5
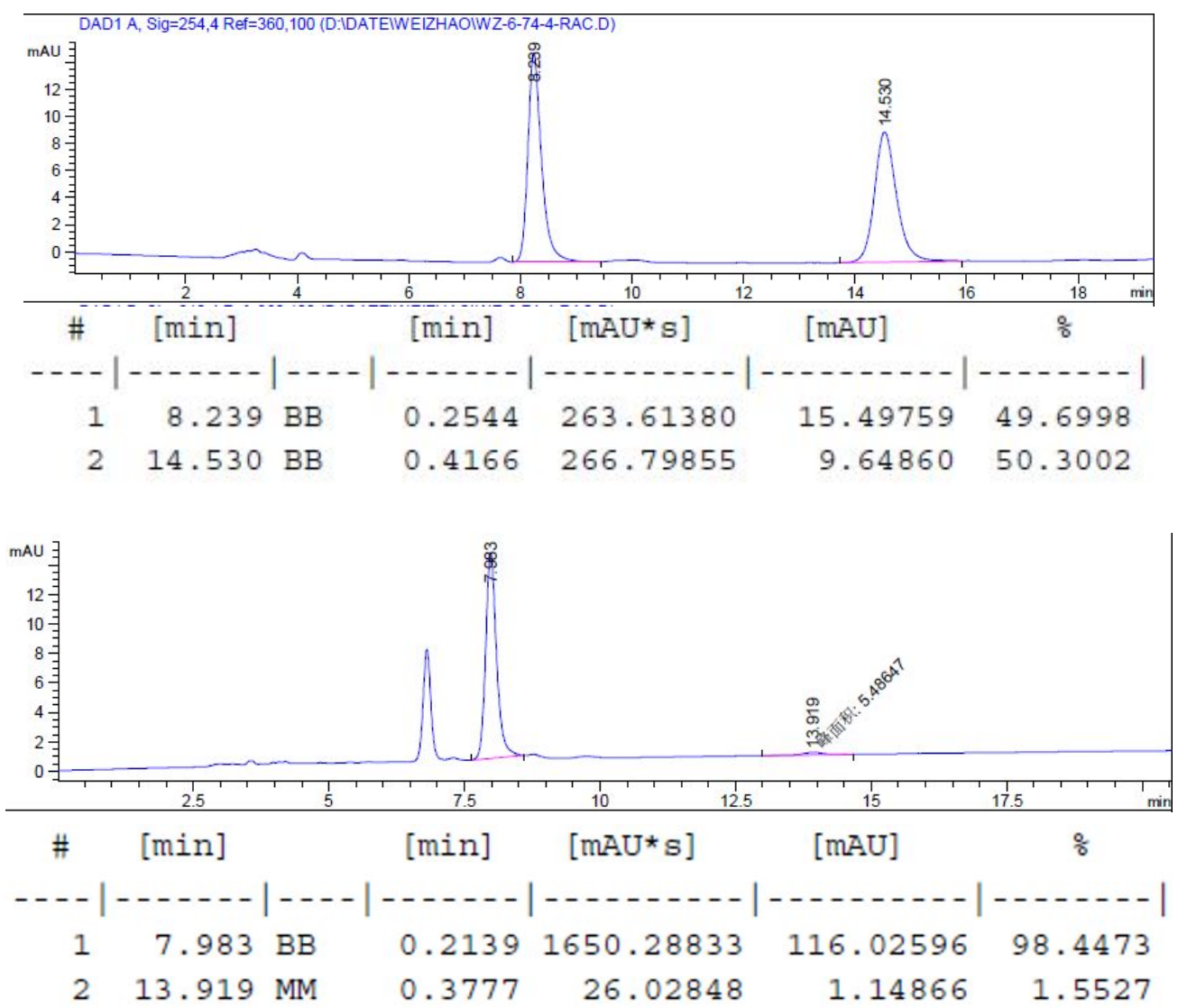


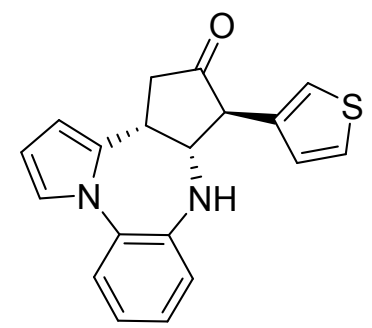

3pa, er 92.5:7.5
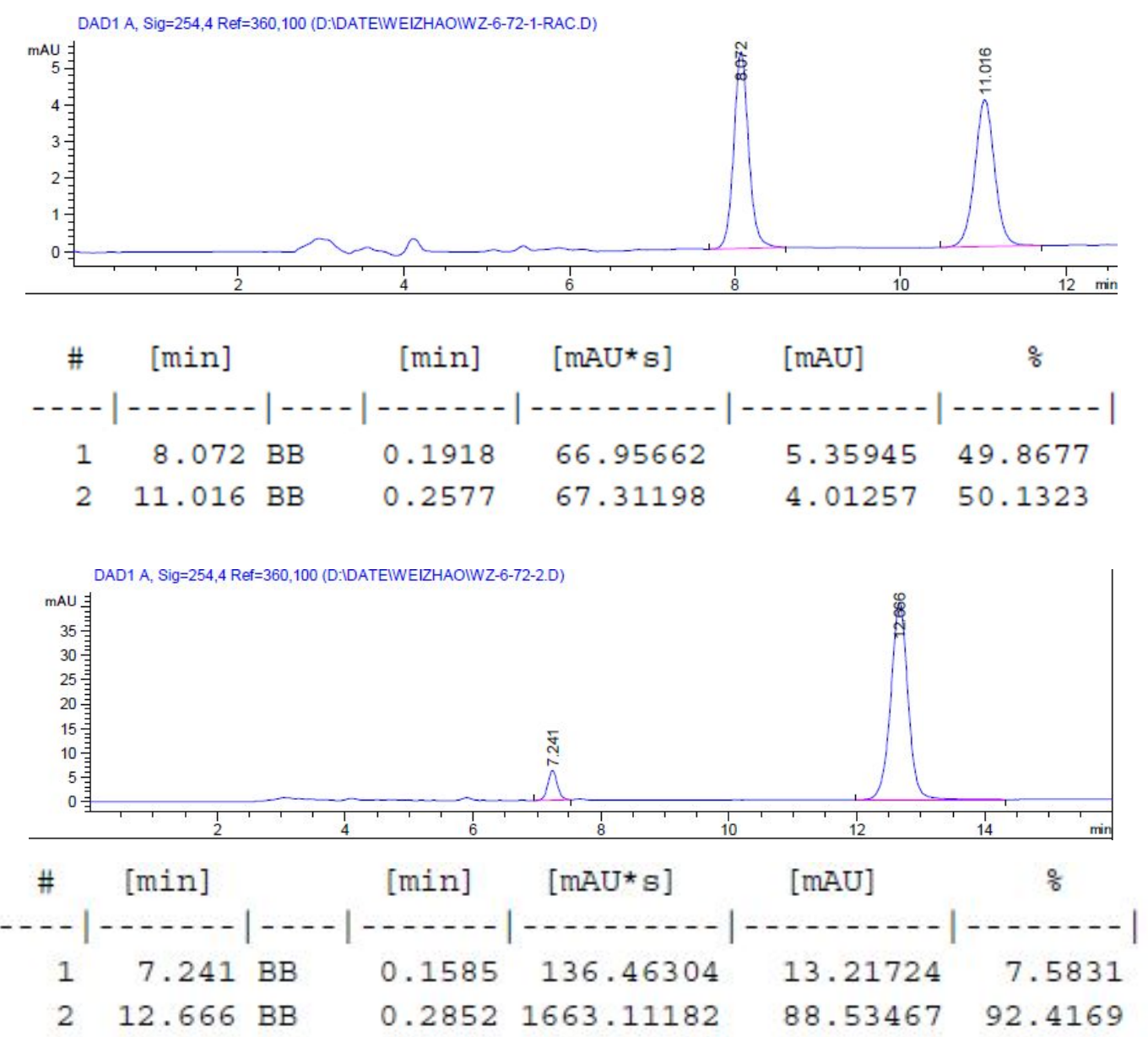

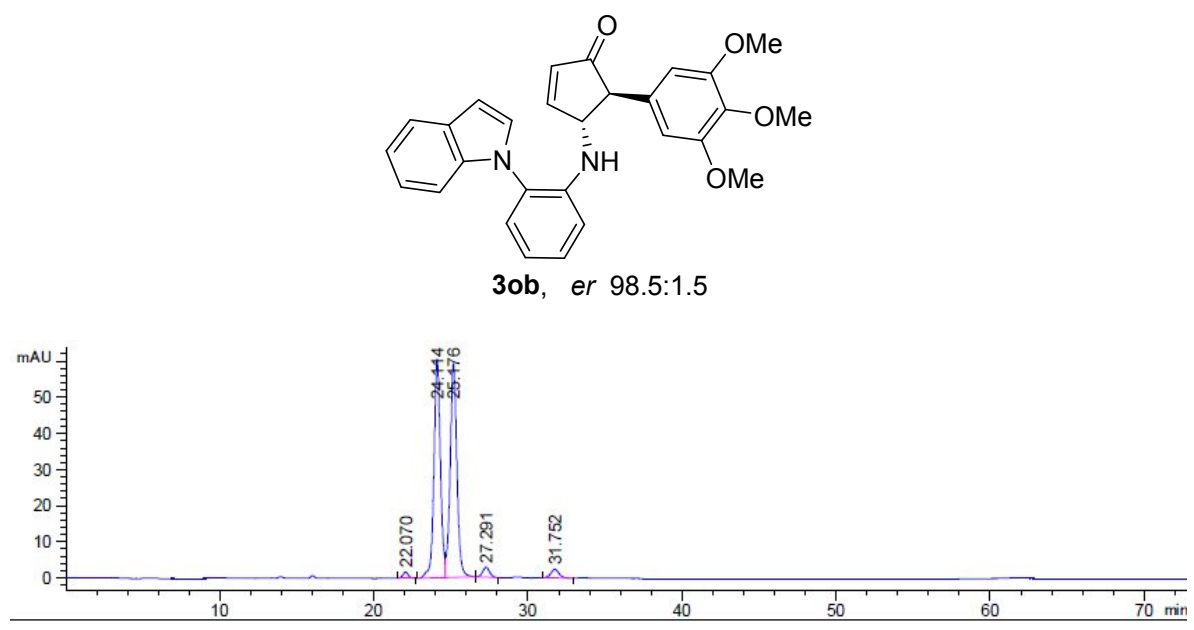

\begin{tabular}{|c|c|c|c|c|c|c|}
\hline \# & [min] & & {$[\mathrm{min}]$} & {$[\mathrm{mAU} * \mathrm{~s}]$} & {$[\mathrm{mAU}]$} & $\%$ \\
\hline & & & 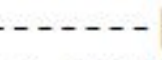 & $-\cdots-\cdots$ & -- & $-1-$ \\
\hline 1 & 22.070 & BB & 0.3757 & 43.03402 & 1. 75721 & 1.1333 \\
\hline 2 & 24.114 & BV & 0.4465 & 1777.84546 & 60.55099 & 46.8206 \\
\hline 3 & 25.176 & VB & 0.4621 & 1805.83508 & 59.17095 & 47.5577 \\
\hline 4 & 27.291 & BB & 0.4821 & 85.58457 & 2.74209 & 2.2539 \\
\hline 5 & 31.752 & $\mathrm{BB}$ & 0.5453 & 84.84242 & 2.33830 & 2.2344 \\
\hline
\end{tabular}

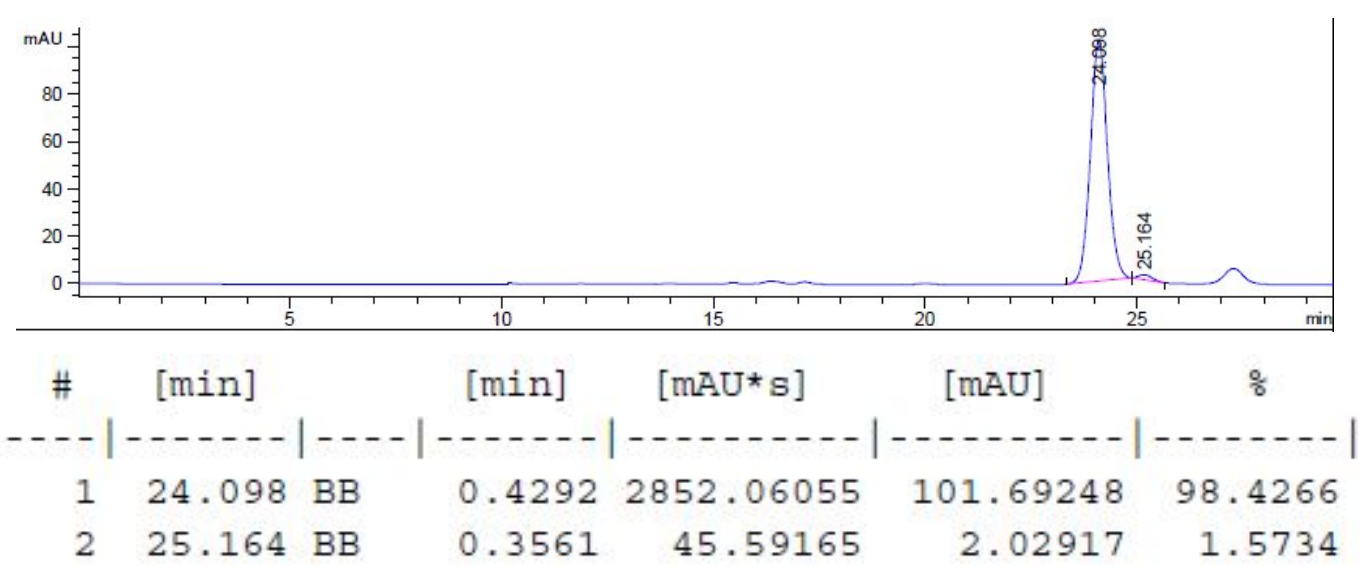




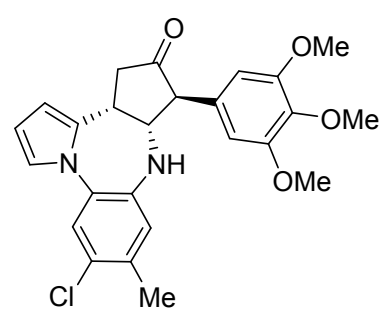

4oc, er 97.5:2.5
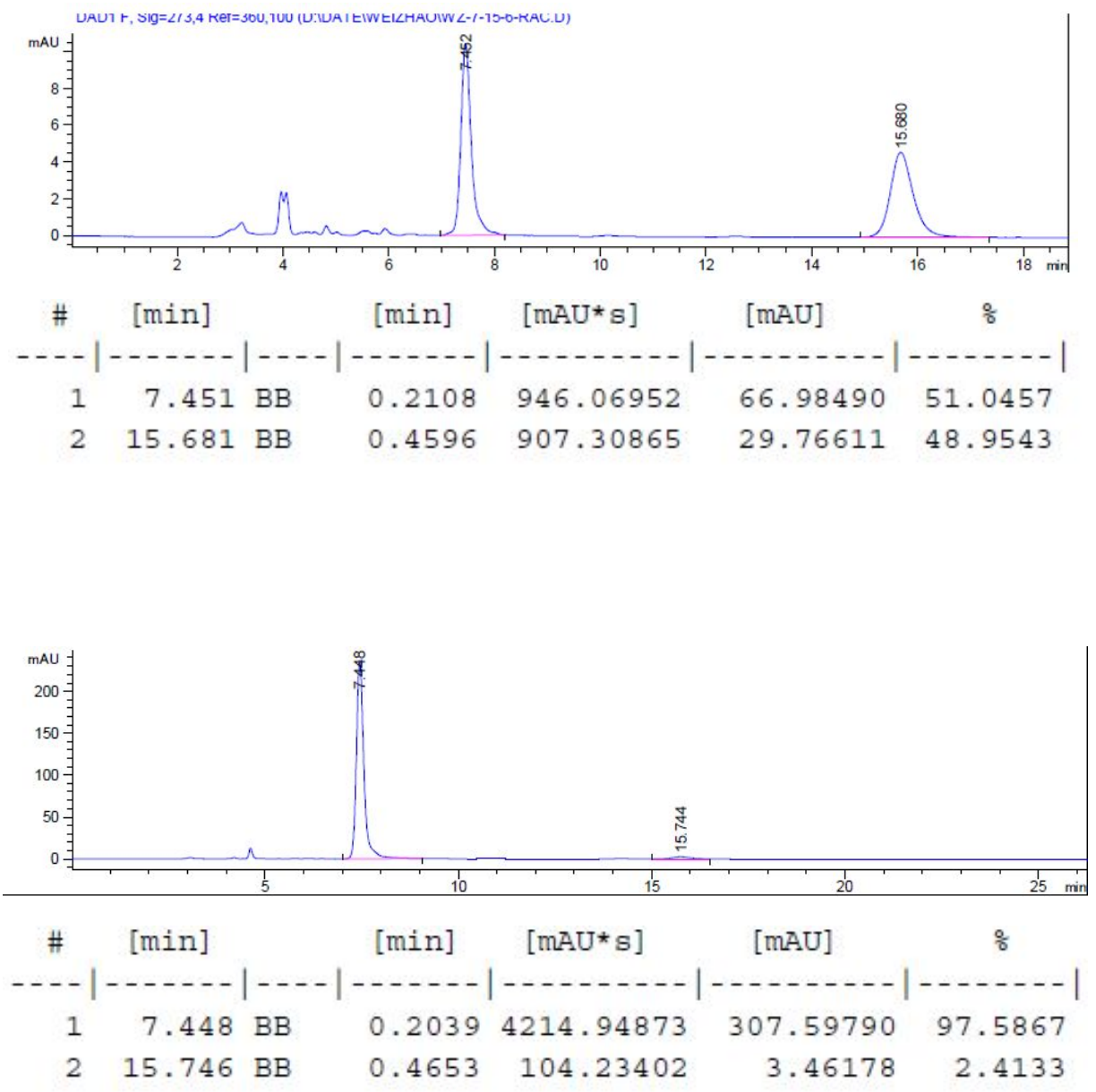


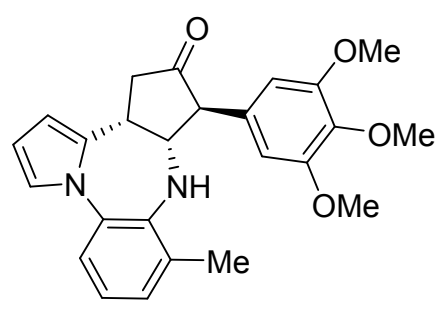

4oe, er 93.5:6.5
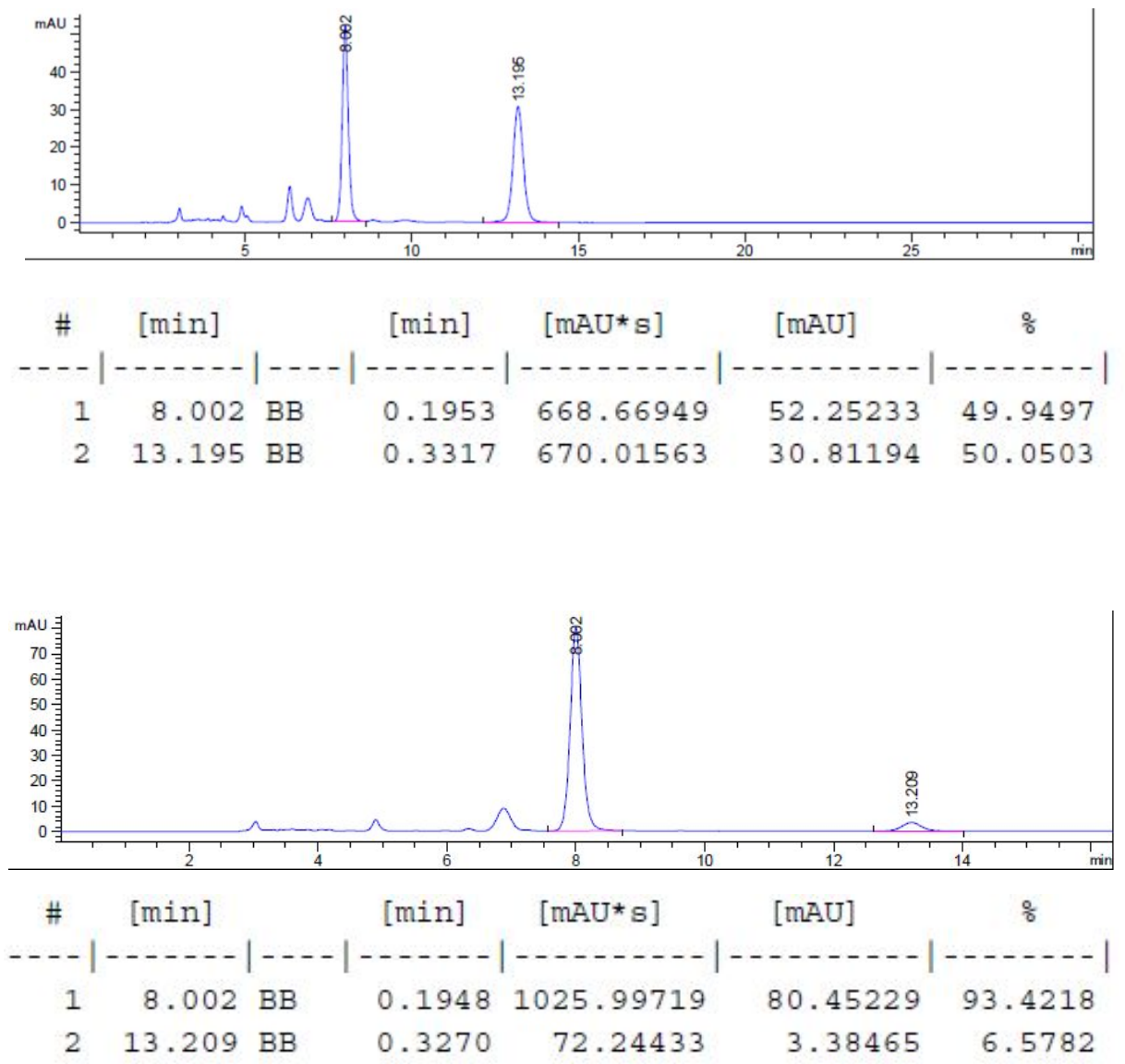

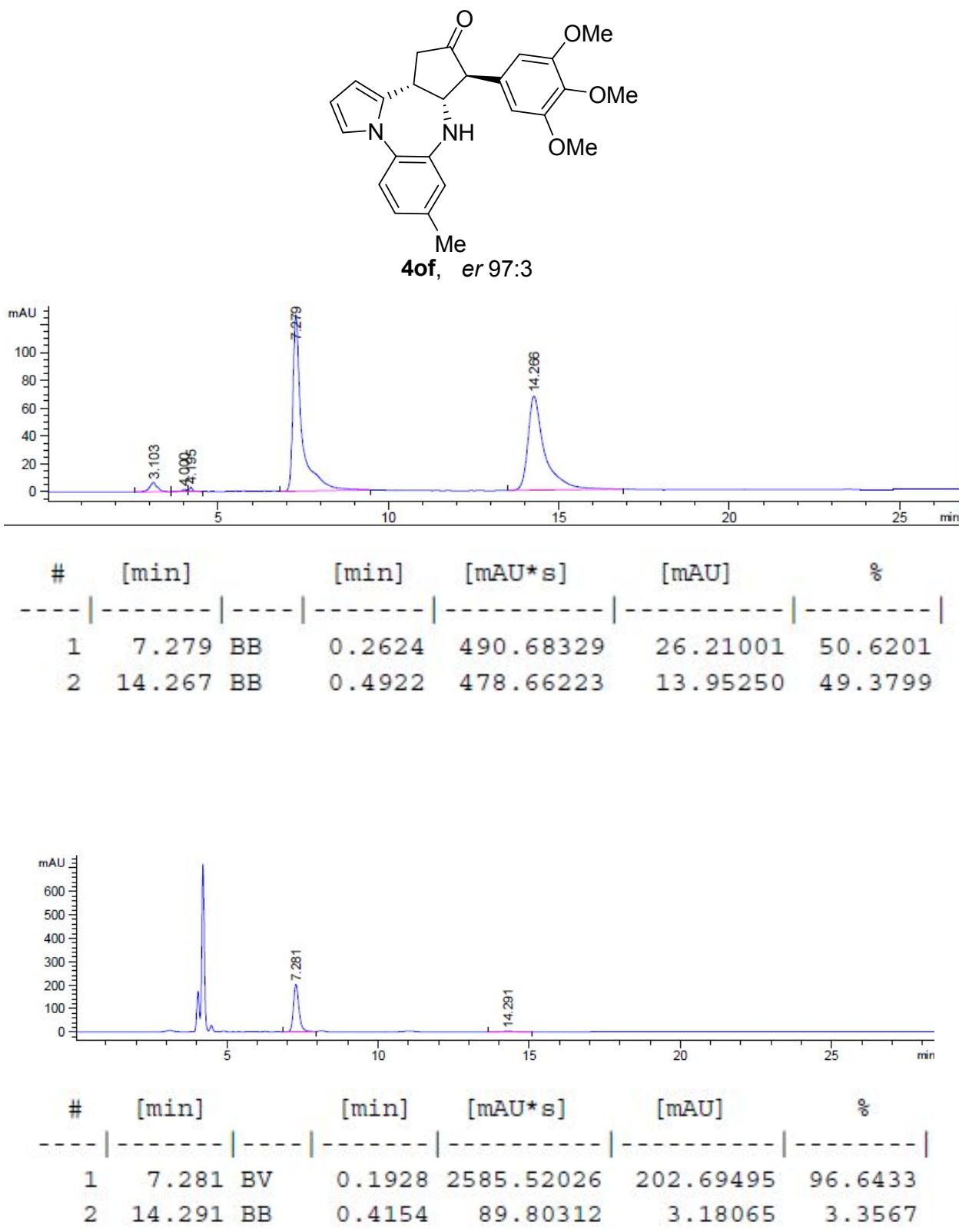

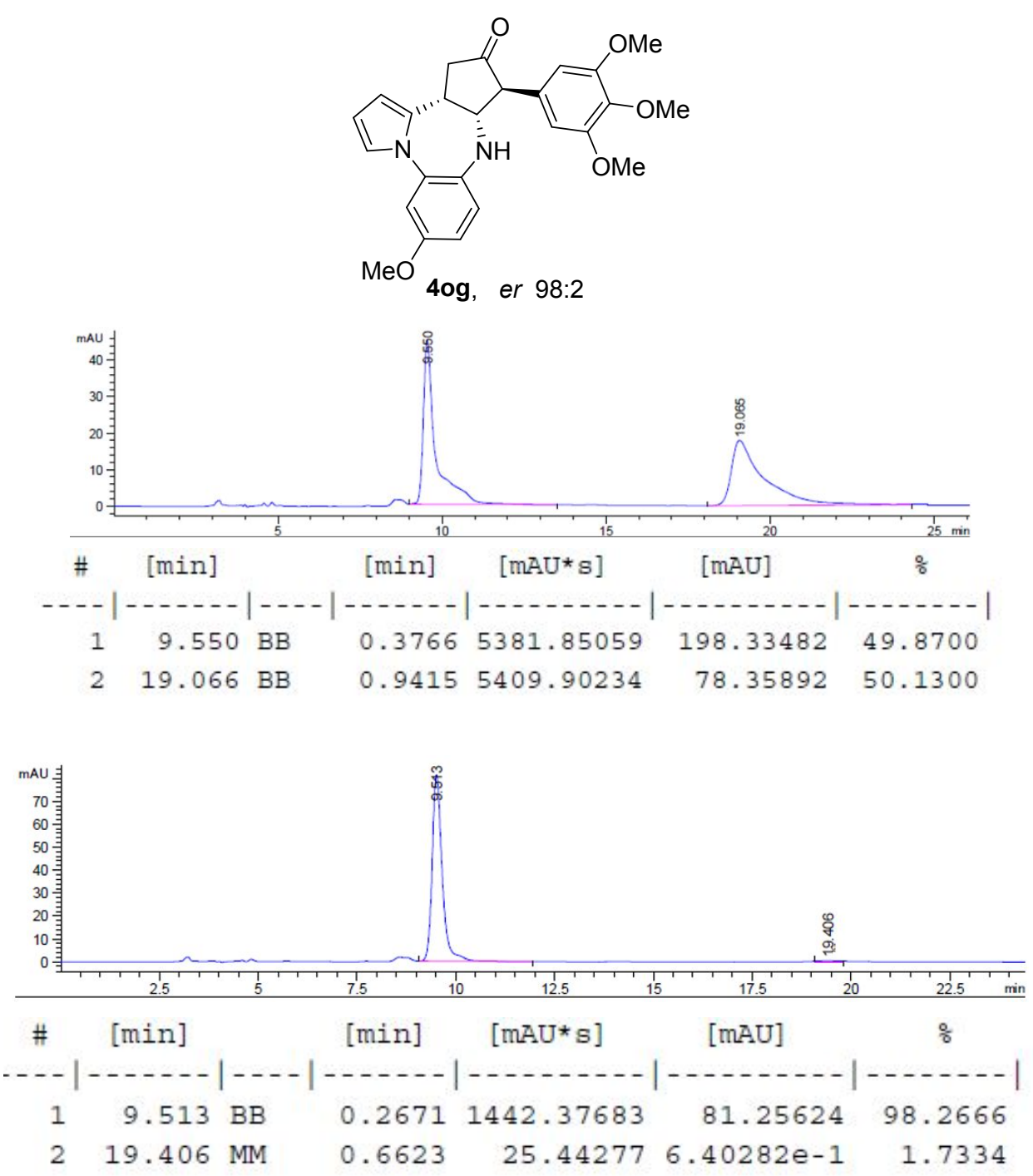

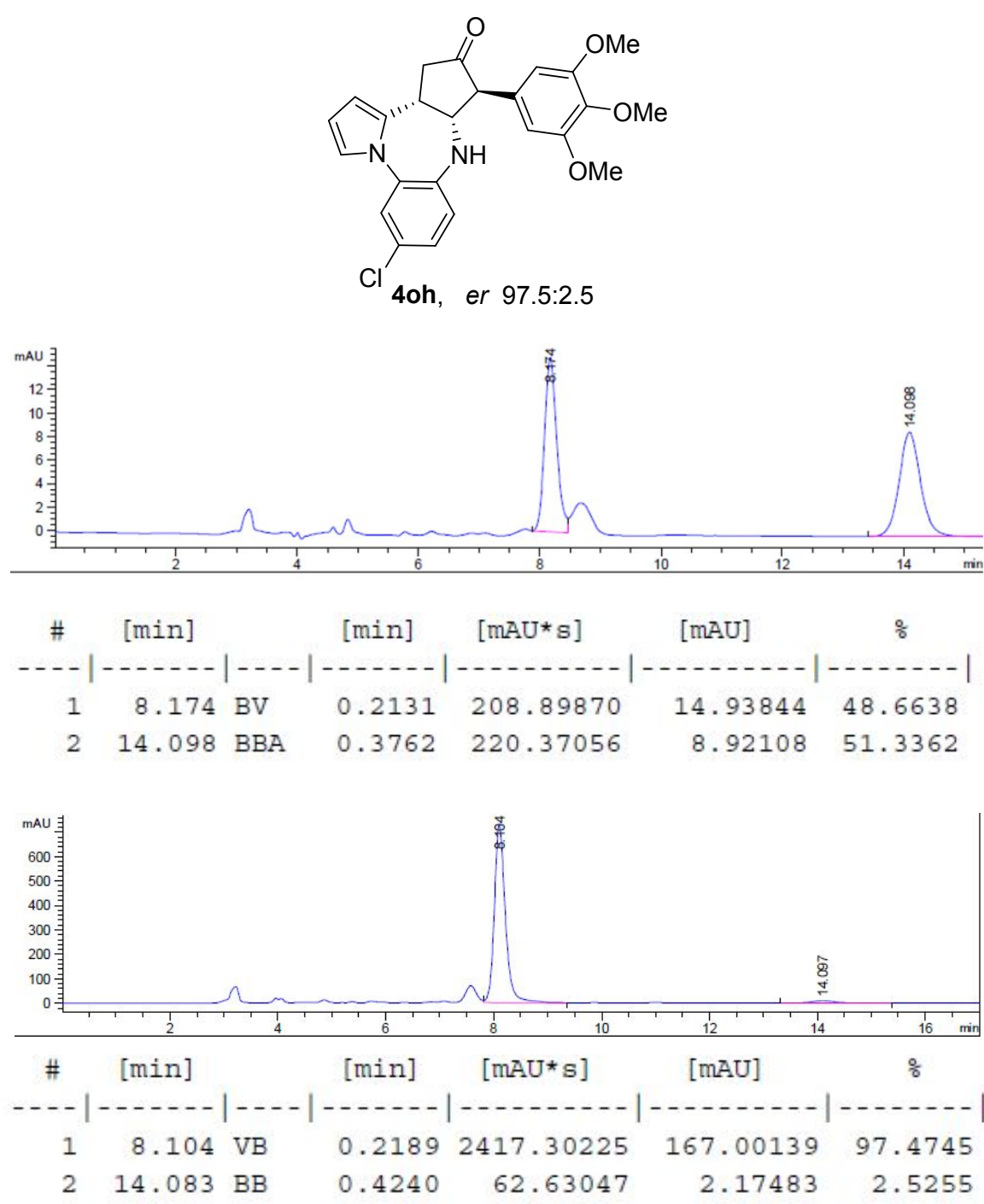

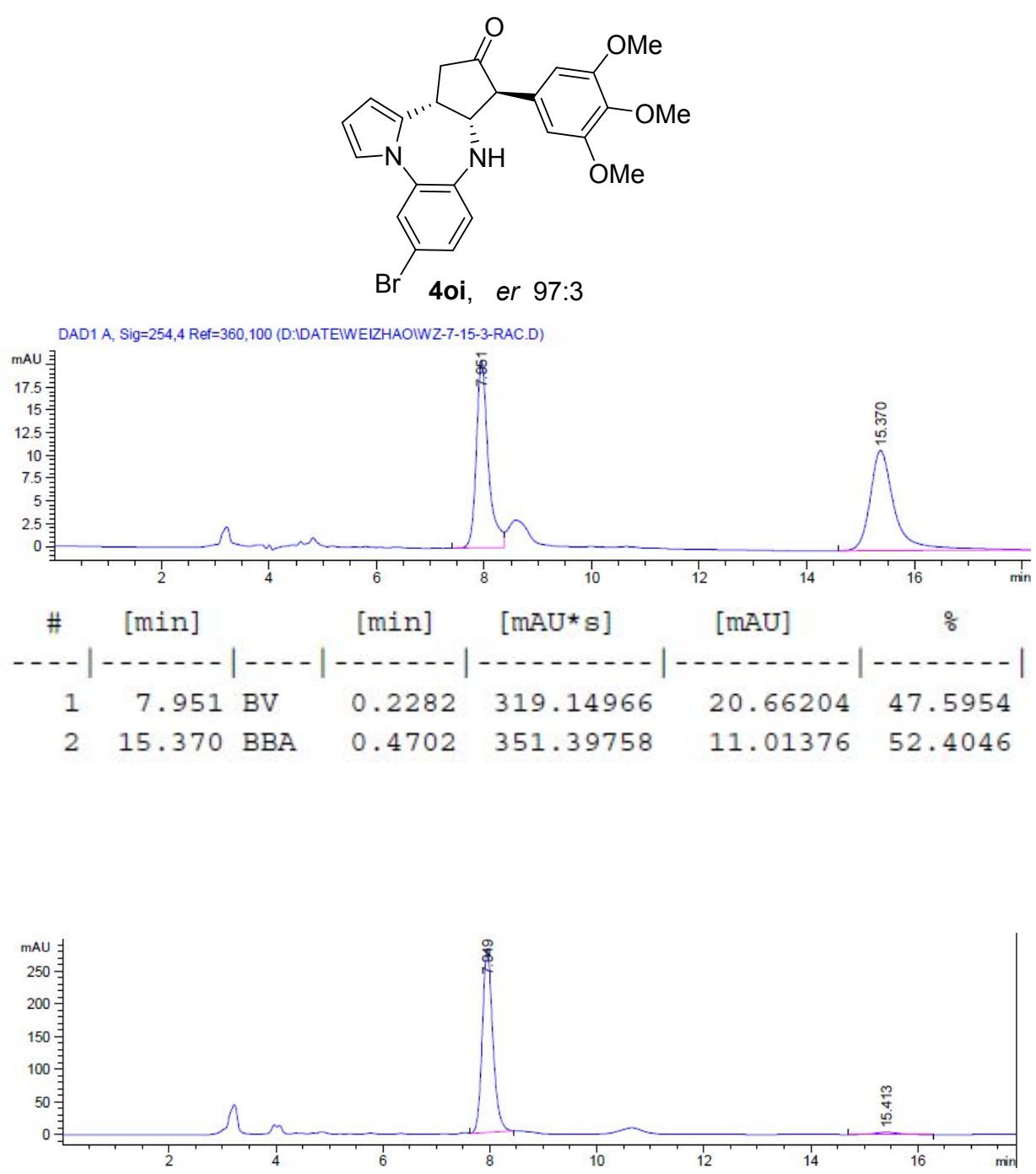

\begin{tabular}{|c|c|c|c|c|c|c|}
\hline \# & [min] & & [min] & {$[\mathrm{mAU} * \mathrm{~s}]$} & [mAU] & $\%$ \\
\hline & & & & & & \\
\hline $\begin{array}{l}1 \\
2\end{array}$ & $\begin{array}{r}7.949 \\
15.413\end{array}$ & $\begin{array}{l}\mathrm{VB} \\
\mathrm{BB}\end{array}$ & $\begin{array}{l}0.2075 \\
0.4278\end{array}$ & $\begin{array}{r}3850.31104 \\
105.95447\end{array}$ & $\begin{array}{r}281.60086 \\
3.74809\end{array}$ & $\begin{array}{r}97.3219 \\
2.6781\end{array}$ \\
\hline
\end{tabular}



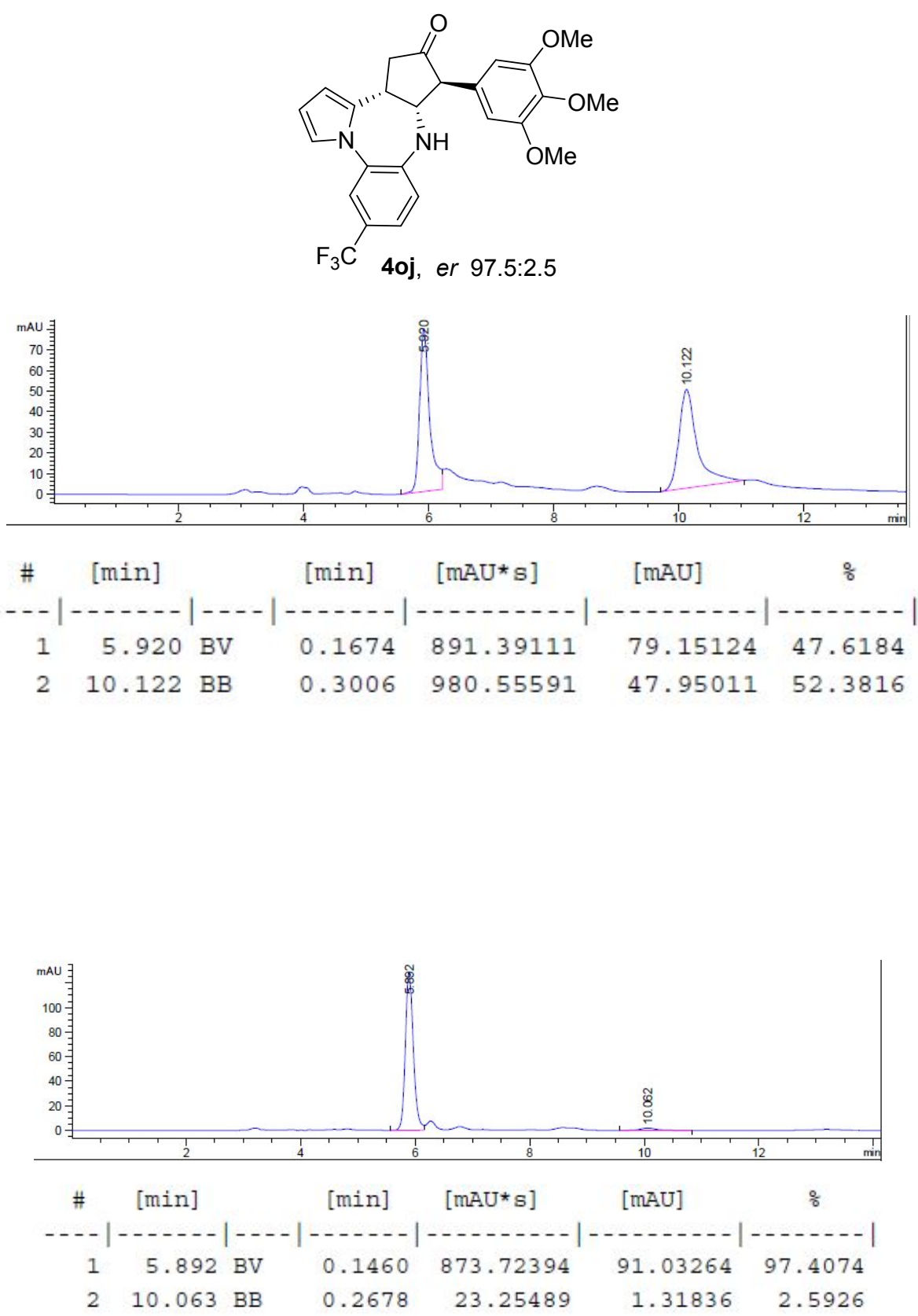

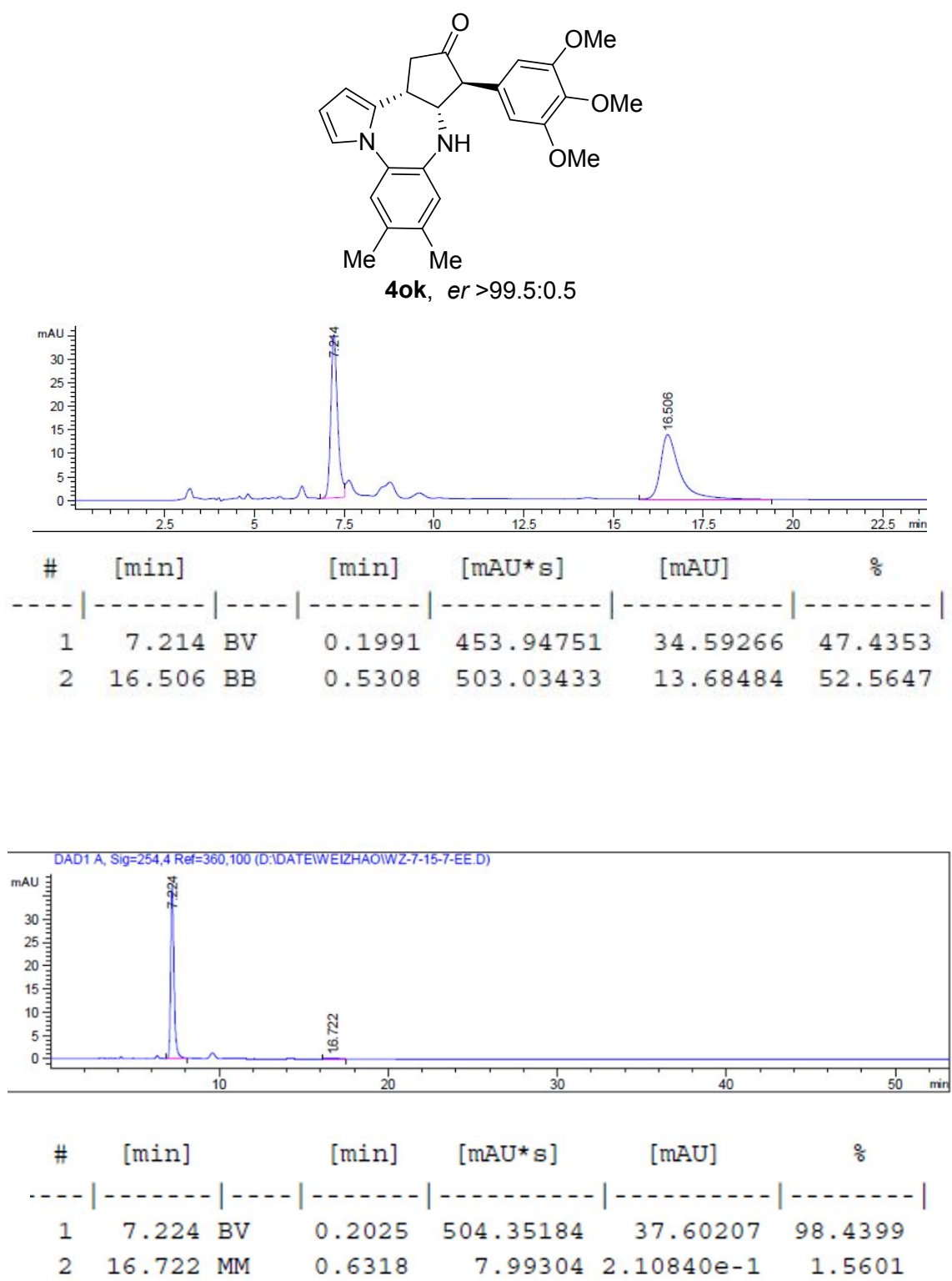

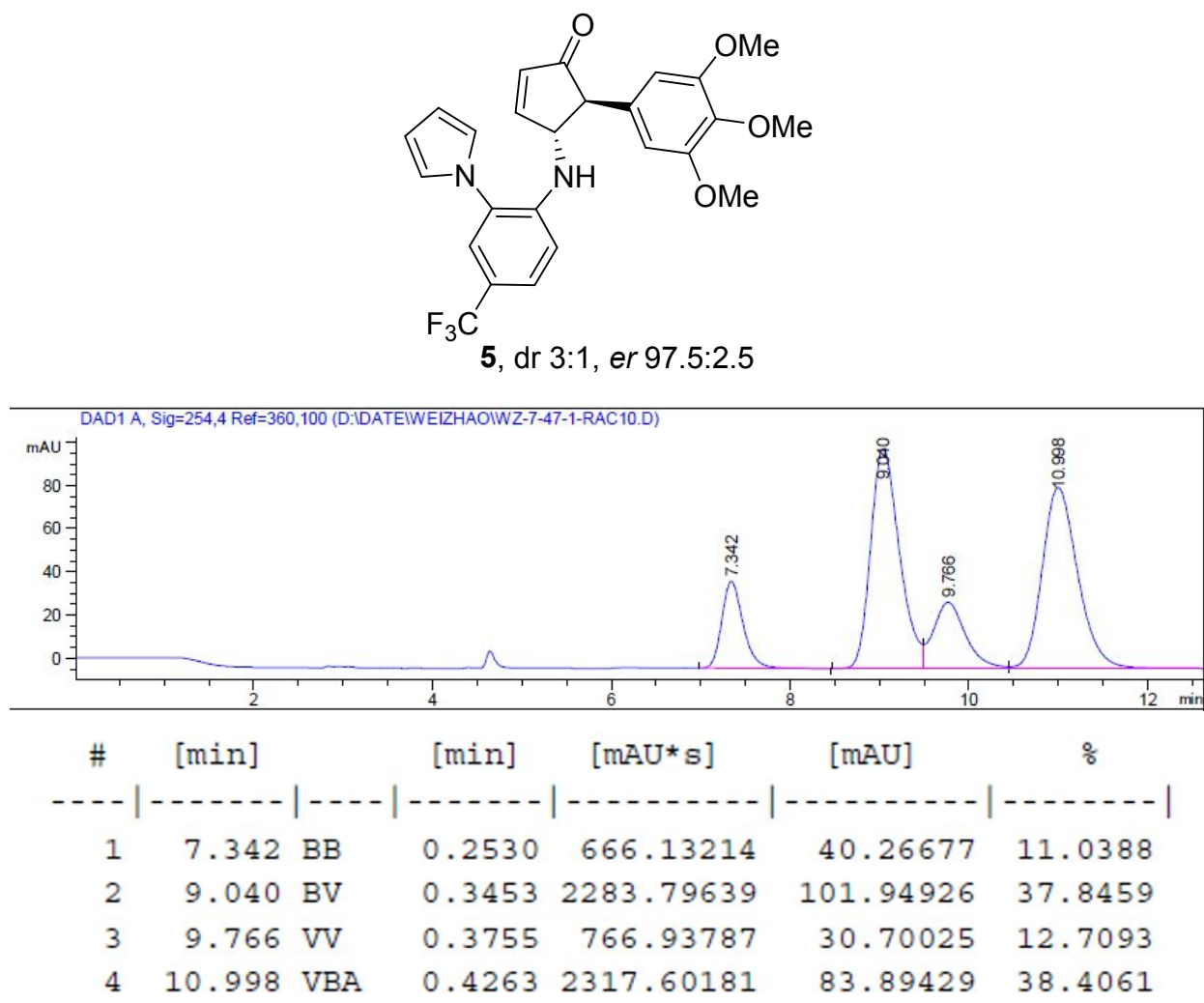

DAD1 B, Sig=210,4 Ref=360,100 (D:IDATEIWEIZHAOIWZ-7-47-1-EERE.D)

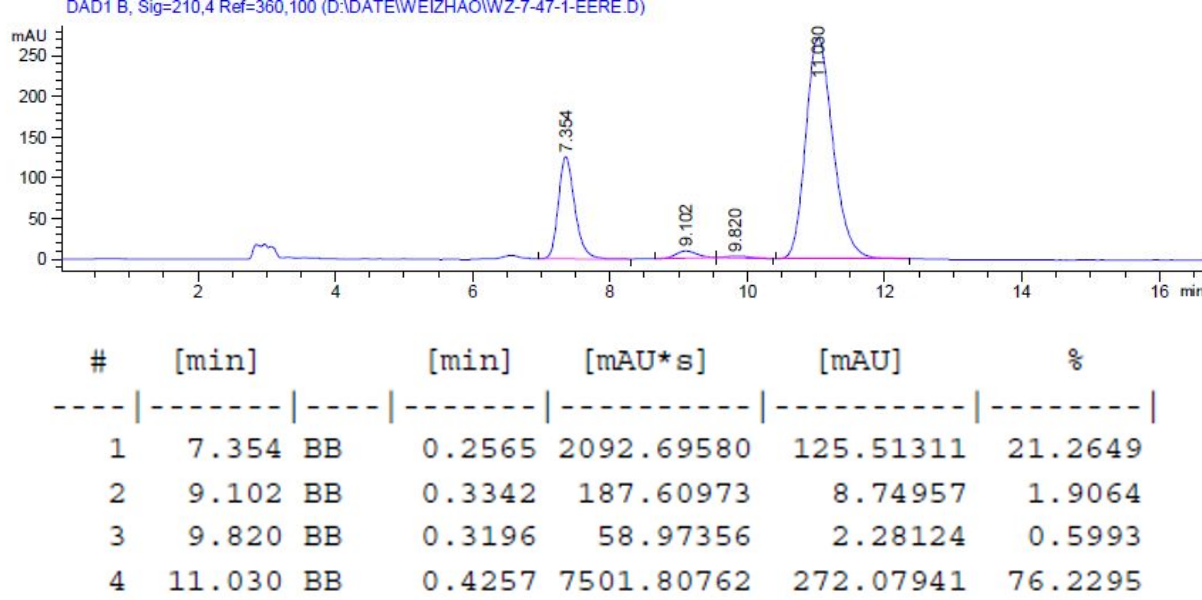




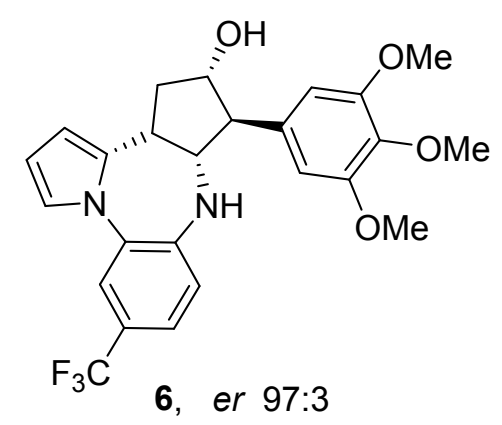

DAD1 A, Sig=254,4 Ref=360,100 (D:IDATEIWEIZHAOIWZ-CF3 REDUCTION RAC.D)

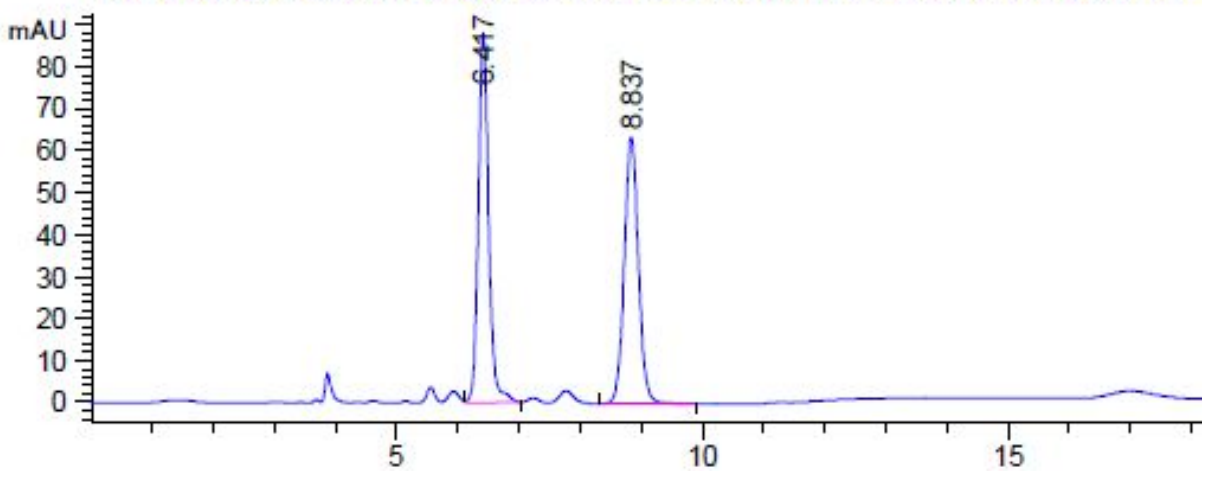

\begin{tabular}{|c|c|c|c|c|c|c|}
\hline$\#$ & [min] & & [min] & {$\left[\mathrm{mAU} U^{*} \mathrm{~s}\right]$} & [mAU] & $\%$ \\
\hline & & & & & & \\
\hline 1 & 6. & VB & 0.1789 & 1034.55640 & 14601 & 50 \\
\hline 2 & 8.837 & BB & 0.2471 & 1019.73981 & 63.59380 & 49 \\
\hline
\end{tabular}

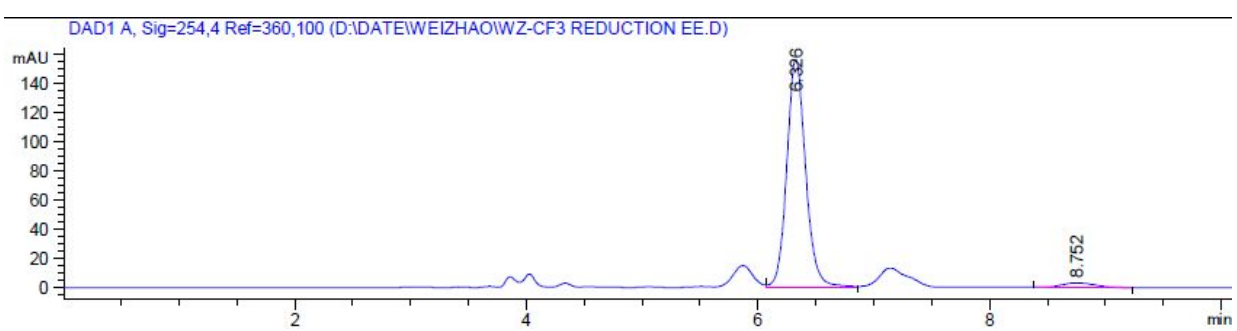

\begin{tabular}{|c|c|c|c|c|c|c|}
\hline$\#$ & {$[\mathrm{~min}]$} & & {$[\mathrm{min}]$} & {$[\mathrm{mAU} * \mathrm{~s}]$} & {$[\mathrm{mAU}]$} & $\%$ \\
\hline & & & & & 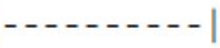 & - \\
\hline 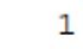 & & VV & & 4345.92920 & 6785 & 97. \\
\hline & 31 & $\mathrm{BB}$ & 50 & 9.99406 & 96319 & 2.6869 \\
\hline
\end{tabular}



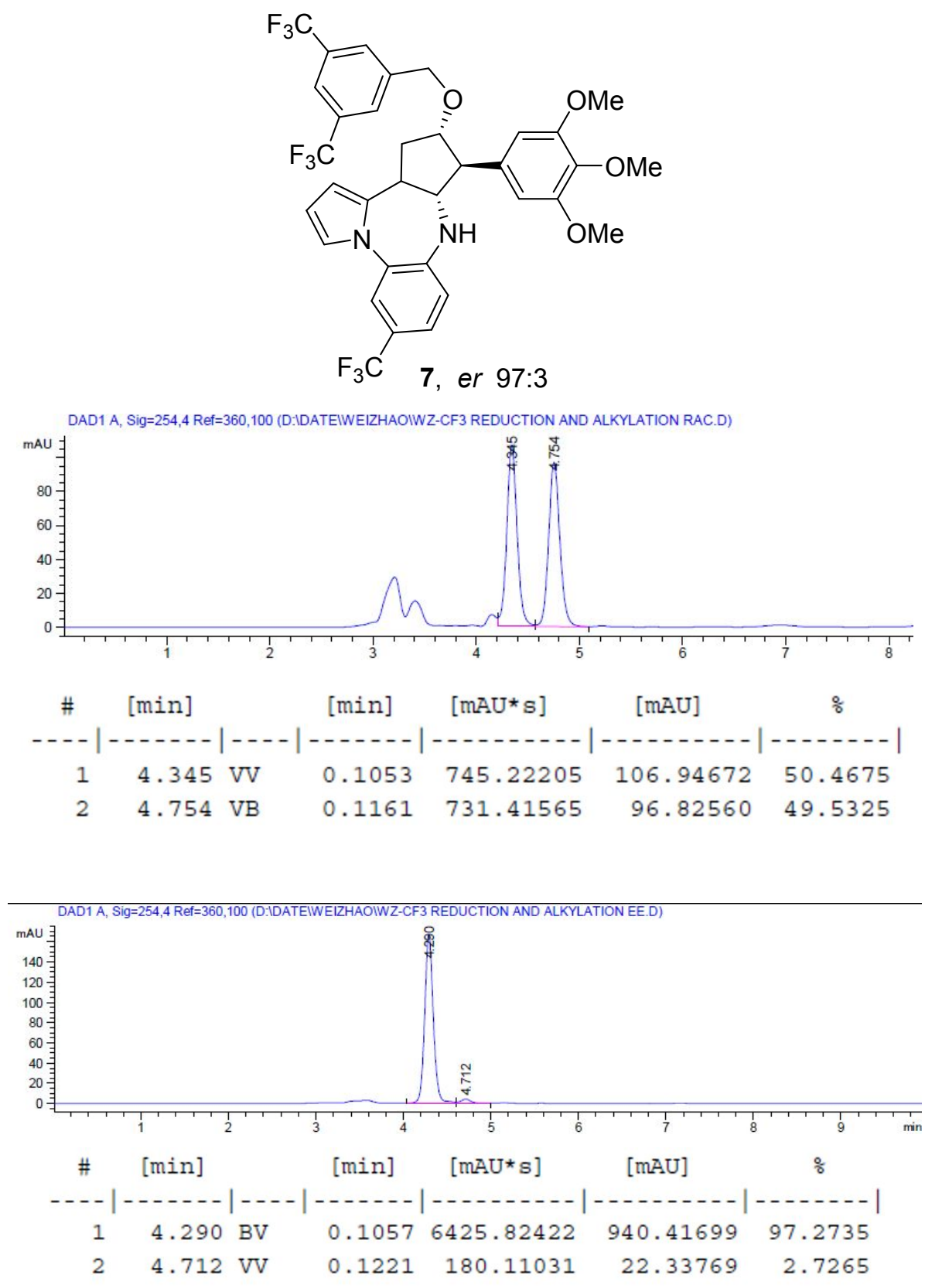